\title{
NEUROGENIC PLASTICITY IN A SEROTONIN TRANSPORTER KNOCKOUT MODEL: EFFECTS OF MATERNAL AND OFFSPRING GENOTYPE
}

BY

\section{SARAH TAPNIKAR}

\begin{abstract}
A thesis submitted to the Victoria University of Wellington in fulfilment of the requirements for the degree of
\end{abstract} Master of Science

Victoria University of Wellington

(2021) 


\section{Table of Contents}

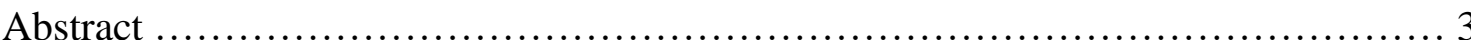

\section{Chapter One}

Serotonin transporter genotypes' effects on neurogenesis

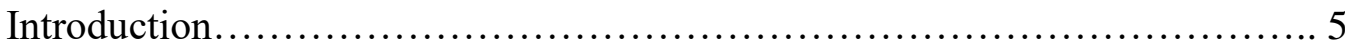

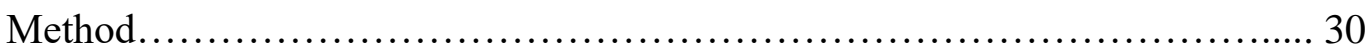

Results................................................................ 36

Discussion............................................................... 42

\section{Chapter Two}

MALDI brain imaging

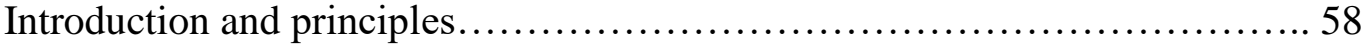

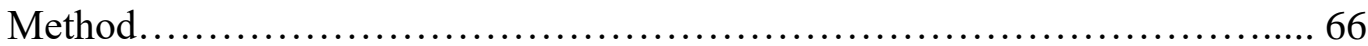

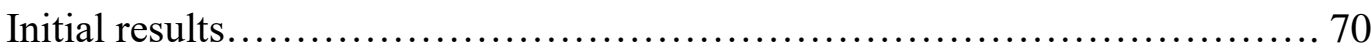

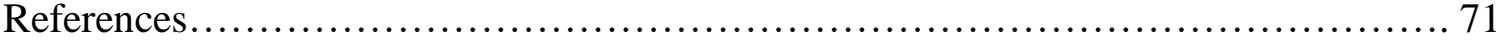

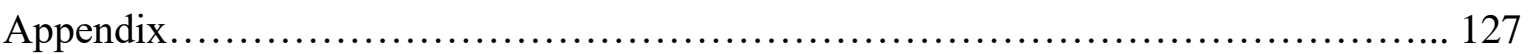




\begin{abstract}
Major depressive disorder (MDD) is debilitating mental disorder that is increasing in prevalence. Many theories have tried to explain the aetiology of depression including the classic monoamine deficiency hypothesis and the newer neurogenic hypothesis. The finding that selective serotonin transporter inhibitors (SSRIs) work by increasing extracellular serotonin levels in the brain and have antidepressant effects has formed the basis of the most widely accepted theory of depression, the monoamine hypothesis. However, a genetic reduction in human and animal serotonin reuptake transporters, which also increases extracellular serotonin, is associated with depressive symptomology. This paradox is not explained by the monoamine hypothesis. The key difference between these two scenarios is that genetically induced increases in serotonin occur from development onward, while SSRIs increase serotonin only in adulthood. Furthermore, SSRIs typically take several weeks to confer a therapeutic effect. This finding has led to the hypothesis that, rather than acute monoamine-increasing effects, it is the downstream effects of such increases on neurogenesis and neural plasticity which confer antidepressant effects. To further elucidate the neurobiology of depression, this study sought to examine the effects of genetically increasing serotonin on early postnatal neurogenesis in a serotonin knockout rat model using BrdU immunohistochemistry. We examined both the offspring and maternal genotype effects. We found that SERT-/- offspring had the highest levels of neurogenesis compared with SERT+/and SERT+/+ at postnatal day 7. In addition we found a maternal genotype effect with SERT+/+ offspring born and reared by SERT+/- mothers having lower neurogenesis compared to SERT+/+ offspring from SERT+/+ mothers. The potential effects of maternal caregiving, neuroplasticity in altered mood and stress responses and the role of 5-HT receptors are discussed.
\end{abstract}


CHAPTER ONE

SEROTONIN TRANSPORTER GENOTYPES' EFFECTS ON NEUROGENESIS 


\section{Introduction}

Depression has become a forerunner in the ailments impacting large amounts of people today. It is a debilitating mental disorder characterised predominantly by a heterogenous spectrum of symptoms including pathologically depressed mood and loss of pleasure, as well as combinations of decreased energy levels, changes in appetite or weight, poor concentration, feelings of guilt or low self-worth and sleep disturbances (American Psychiatric Association [APA], 2013). It is highly prevalent, with the World Health Organization estimating that over 300 million people suffer from depression and that this rate is increasing globally (World Health Organization [WHO], 2017).

The lifetime prevalence of depression varies across countries with the rate in New Zealand at 1.5 cases per 100 adults and a mean age of onset of 18.2 years (Simon, Goldberg, Kroff, Üstün, 2002; Weissman et al., 1996). The diagnostic and statistical manual of mental disorders (DSM) puts the lifetime risk of major depressive disorder at 10-25\% for women and $5-12 \%$ for men while the point prevalence is between $5-9 \%$ in women and $2-3 \%$ in men (APA, 2013). The lifetime prevalence rate appears to be on the rise and occurring earlier in life (Joyce, Oakley-Browne, Wells, Bushnell \& Hornblow, 1990). In many countries, mood disorders are the second most common type of mental illness after anxiety disorders (Demyttenaere et al., 2004). The prevalence of depression in adolescents and young adults has increased recently (Mojtabai, Olfson \& Han, 2016) and the total estimated number of people living with depression increased by $18.4 \%$ from 2005 to 2015 (Vos et al., 2016).

The gravest consequence of depression is unquestionably suicide. Overall, depression increases the risk of suicide ideation and attempt, as well as death by suicide (Ribeiro, Huang, Fox \& Franklin, 2018). Studies find mortality rates of suicide to be up to 27 -fold greater for those with affective disorders more generally, as well as depression specifically, when compared with the general population (Bostwick \& Pankratz, 2000; Melchior et al., 2010; Ösby, Brandt, Correia, Ekbom \& Sparén, 2001). The mortality risk associated with depression also increases with the severity of symptoms (Almeida, Alfonso, Hankey \& Flicker, 2010). Antidepressant use is also associated with a $30 \%$ higher risk of mortality and this association is independent of the presence of clinical depression (Almeida et al., 2010). Suicide accounted for $1.5 \%$ of all deaths in 2015 bringing suicide into the top 20 leading causes of death (although many more attempts are made; WHO, 2017). 
Higher levels of impairment are reported for mental disorders (particularly anxiety, mood and impulse control disorders) than other chronic medical conditions, with severe functional impairment reported by $42 \%$ of those with mental disorders and $24 \%$ with chronic medical disorders (Druss et al., 2009). While prevalence rates differ drastically across countries, depression is universally associated with disability (Simon et al., 2002). However, treatment is provided for a significantly lower proportion of mental disorders than medical conditions (Druss et al., 2009). Psychosocial and relationship impairment, in particular, is most commonly associated with mood disorders (compared with chronic medical conditions that are associated more with work and home functioning; Druss et al., 2009; Leader \& Klein, 1996; Lee \& Murray, 1988) and is associated with depressive symptom severity (Judd et al., 2000). While treatment improves functional impairment, some level of disability appears to persist even after remission (Kennedy, Foy, Sherazi, McDonough \& McKeon, 2007; Mintz, Mintz, Arruda \& Hwang., 1992). Longitudinal studies have also found that up to one-third of depressed patients die or suffer chronic incapacitation (Brodaty, Luscombe, Peisah, Astney \& Andrews, 2001; Kiloh, Andrews \& Neilson, 1988; Lee \& Murray, 1988; Surtees \& Barkley, 1994). Chronic depression is more common than initially thought with up to $21 \%$ of depressed patients suffering chronically (Gilmer et al., 2005), and Kocsis et al. (2008) found that the mean duration of chronic depression was 20 years. Chronic depressive episodes were also associated with greater illness burden, comorbidity and severity (Gilmer et al., 2005; Kessler, Zhao, Blazer \& Swartz, 1997). Depression is a recurrent illness as longitudinal studies reveal that up to $86 \%$ of patients in remission undergo relapse (Brodaty et al., 2001). Older studies found even higher relapse rates of up to 95\% (Kiloh et al., 1988; Lee \& Murray, 1988; Mueller et al., 1999).

In terms of work-related disability, Broadhead, Blazer, George and Tse (1990) report that depressed individuals have up to 4.78 times higher risk of absenteeism and daily task impairment than asymptomatic individuals. Depressive symptomology is also associated with increased unemployment and decreased annual salary with studies finding annual income losses due to absenteeism and presenteeism of \$4400 and \$9600 USD for depressed and bipolar individuals respectively (Kessler et al., 2006; Whooley et al., 2002). Loss projections in the US estimate a $\$ 36.6$ billion salary-equivalent lost productivity per year due to major depressive disorder (Kessler et al., 2006). Clearly depression is a disabling condition causing significant impairments in an individual's social functioning, work and for the wider society (Briley \& Moret, 2010). 


\section{The Monoamine Hypothesis}

Understandably, the neurobiological aetiology of depression is of great interest. The monoamine hypothesis is one of the most widely accepted theories of depression and has informed the use of most currently-used antidepressant medications (Boku, Nakagawa, Toda \& Hishimoto, 2018).

The monoamine deficiency hypothesis of depression (aka the biogenic amine hypothesis) proposes that depressive and anxious symptoms arise from insufficient levels of the monoamine neurotransmitters serotonin (5-HT), norepinephrine (NE) and dopamine (DA; Massart, Mongeau \& Lanfumey, 2012; Tran, Bymaster, McNamara \& Potter, 2003). It arose from studies showing that the depletion of monoamines results in an induction of depressive symptomology while antidepressant drugs, such as selective serotonin reuptake inhibitors (SSRIs) and monoamine oxidase inhibitors (MAOIs), increase monoamine levels and alleviate depression (Belmaker \& Agam, 2008; Coppen, 1967; Gingrich \& Hen, 2001; Hamon \& Blier, 2013; Holschneider et al., 2001; Salomon et al., 2003; Ruhé et al., 2000; Wong \& Licinio, 2001). The SSRIs mode of action is a rapid and immediate increase in synaptic 5-HT availability while chronic treatment downregulates the serotonin transporter (SERT, aka 5-HTT) and the autoreceptor 5-HT1A and heteroreceptor 5-HT1B, although other 5-HT receptors are also involved in the antidepressant effects of SSRIs (Blier \& Ward, 2003; Cryan, Valentino \& Lucki, 2005; Descarries \& Riad, 2012; Fabre et al., 2000; Gray et al., 2013; Hervás et al., 2001; Le Poul et al., 1995, 2000; Lesch et al., 1993; Moret \& Briley, 2000; Nishi \& Azmitia, 1999). There is also an association between reduced (or a complete lack of) 5-HT1A and 5-HT1B's inhibitory regulation of serotonergic signalling with depressive outcomes which lends further support for the notion of a group which is genetically vulnerable to depression due to a genetic dysregulation of their serotonergic system (Albert \& Lemonde, 2004; Boldrini, Underwood, Mann \& Arrango, 2008; GarciaGarcia et al., 2014; Lemonde et al., 2003; López-Figueroa et al., 2004; Richardson-Jones et al., 2011; Xia et al., 2012; Zhuang et al., 1999).

Overall, these studies suggest an abnormality of serotonergic function in depression where a deficit leads to depression for which antidepressants can help in compensating (Neumeister, 2003). 
One aspect of the monoamine hypothesis that remains unexplained is that, while antidepressants have an acute biochemical action in increasing monoamine levels, there is a delay between this and their clinical/ therapeutic effect of approximately four weeks or more (Hindmarch, 2001; Sapolsky, 2004; Wong \& Licinio, 2001). Moreover, many depressed individuals are not responsive to antidepressants (Serretti et al., 2004). Thus, it has been argued that secondary, adaptive changes in the brain are underlying the clinical improvements rather than the primary/acute serotonin enhancing effect of the drug (Hindmarch, 2001). The focus of current research is investigating the underlying molecular and cellular mechanisms that occur due to downstream changes in the brain after chronic antidepressant treatment.

As 5-HT has also been shown to be a potent regulatory growth signal during neurodevelopment and facilitates neurogenesis in adulthood (Vitalis \& Panavelas, 2003), one proposed downstream antidepressant mechanism is neurogenesis and changes in neuroplasticity. A multitude of studies show that SSRIs facilitate neurogenesis, increase neurotrophic factors' expression, and alter neural circuitry, mainly via an increase in extracellular 5-HT levels (Cai et al., 2013; Surget et al., 2008).

Another potential criticism of the monoamine hypothesis which is in sharp contrast to the beneficial effects of SSRIs in adulthood is that animal and human research also indicates that early developmental increases in 5-HT (either by pharmacological or genetic means) enhances anxiety, depression and emotional problems in later life (Ansorge, Morelli \& Gingrich, 2008; Lira et al., 2003; Oberlander, Gingrich \& Ansorge, 2009, Oberlander et al., 2010). These tenets of the monoamine hypothesis illustrate that the effects of 5-HT are multifaceted and need further study.

\section{The SLC6A4 serotonin transporter gene}

Early heritability studies have confirmed a genetic component of depression (Hamet $\&$ Tremblay, 2005; Shadrina et al., 2018). Monoaminergic transporters have been of particular interest because they are sites of action for most antidepressants as well as for certain psychostimulants (Borowsky \& Hoffman, 1995). The serotonin transporter has a critical role in serotonergic neurotransmission and homeostasis as it is responsible for the active transport (sodium-dependent reuptake) of 5-HT back into the presynaptic neuron to reduce synaptic concentrations of 5-HT and terminate post-synaptic transmission (Murphy et al., 2004; Rudnick, 1977; Rudnick \& Clark, 1993). Research into the genetic determinants of 
depression has since focused heavily on a serotonin transporter polymorphism's (5-HTTLPR) contribution to the aetiology for depression (Cervilla et al., 2006; Furlong et al., 1998; Gutiérrez et al., 1998).

Within the human SERT gene's (termed solute carrier 6a4, SLC6A4) promoter region is a 5-HTT-linked polymorphic region (5-HTTLPR) which has an insertion/deletion variable nucleotide repeat (VNTR) polymorphism with a short ( $s$ ) allele consisting of a $\sim 44$ base pair (bp) deletion of repeat elements 6-8 from bp 1,212 to bp 1,255 (Heils et al., 1996; Kraft, Slager, McGrath \& Hamilton, 2005; Lesch et al., 1996; Wendland, Martin, Kruse, Lesch \& Murphy, 2006). Thus, the 5-HTTLPR consists of a length variation of a repetitive sequence containing 20-23-bp-long GC-rich segments with 14 repeats in the short allele and 16 in the long allele (Heils et al., 1996).

This is a functionally significant polymorphism as these two common alleles of the SERT gene promoter region polymorphism impact its transcriptional activity (Heils et al., 1996; Lesch et al., 1996). The long ( $l$ ) allele confers threefold greater transcriptional activity than the short allele which has reduced SLC6A4 mRNA production and SERT binding (Bradley, Dodelzon, Sandhu \& Philibert, 2005; Heils et al., 1996; Lesch et al., 1996; Stoltenberg et al., 2002). In humans, lymphoblast cells homozygous for the $l$ 5-HTTLPR variant produced 1.4 to 1.7 times more SLC6A4 mRNA, had 30-40\% more SERT proteins and had 1.9-2.2 times more 5-HT uptake than cells containing one or two copies of the $s$ variant (Lesch et al., 1996). The $s$ allele variant also has reduced enhancer/promoter repression compared to the $l$ variant (Heils et al., 1996).

Importantly, this polymorphism has been found to be associated with neuropsychiatric disorders, in particular the $s$ allele is associated with a greater risk of developing affective and mood disorders (Furlong et al., 1998; Gutiérrez et al., 1998) and psychological traits such as neuroticism (Lesch et al., 1996). Naturally, because of its crucial role in serotonergic homeostasis as well as being the site of action of many antidepressants, the SLC6A4 gene is a candidate for mood disorder aetiology (Kelsoe et al., 1996). It is important to note, however, that in addition to the $l$ and $s$ 5-HTTLPR variants, there are many more SLC6A4 polymorphisms (both in the 5-HTTLPR itself, as well as in other regions of the gene). Several of these, which affect SERT levels, are also associated with affective disorders (Hu et al., 2005; Jarrett et al., 2007; Kraft et al., 2005; Ogilvie et al., 1996; Wendland et al., 2006). 
Mounting evidence from studies of depressed individuals reveal a multitude of serotonergic system changes that occur in comparison to healthy controls (D'haenen, 2001). For example, studies have demonstrated decreased SERT binding sites in the brain of depressed individuals (Ellis \& Salmond, 1994; Malison et al., 1998; Mann et al., 2000; Owens \& Nemroff, 1994), reduced concentrations of the major 5-HT metabolite 5hydroxyindoleacetic acid (5-HIAA) in depressed individuals and suicidal patients (Gibbons \& Davis, 1986; Roy, Jong \& Linnoila, 1989), decreased tryptophan concentrations in depressed patients (Quintana, 1992) which also led to rapid relapses/ return of symptoms in depressed patients (Morris et al., 1999; Neumeister et al., 2004).

Research into the 5-HTTLPR polymorphism's significance for depression, however, has been less consistent. Initially several studies found that the $s$ allele is associated with a higher risk for developing affective disorders, particularly depression (Cervilla et al., 2006; Furlong et al., 1998; Gutiérrez et al., 1998). Cervilla et al. (2006) found that the association between the $s / s$ genotype and depressive outcomes was stronger for more severe depression. The 5-HTTLPR $s$ allele was also found to occur more in patients with Seasonal Affective Disorder (SAD) than controls (Rosenthal et al., 1998). SAD patients were less likely to have the $l / l$ genotype but those that did had lower seasonality scores than patients with $s / l$ or $s / s$ genotypes (Rosenthal et al., 1998). The 5-HTTLPR $s / s$ genotype is also associated with a poorer response to SSRI treatment than $s / l$ and $l / l$ genotypes, with the $s / s$ genotype being associated with greater difficulties reaching remission and a lower response rate (Serretti et al., 2004, Serretti, Kato, De Ronchi \& Kinoshita, 2007). On the other hand, there have been recent studies and meta-analyses that have been unable to confirm the association of the $s$ allele with depression. Most recently a large population-based study involving over 100,000 participants found no evidence of a linkage between the $s$ allele of the 5-HTTLPR and depression (Border et al., 2019). While all these data strongly implicate the serotonergic system in the pathophysiology of depression, its exact role and the genetic contribution of SLC6A4 in particular remain to be fully elucidated.

\section{The serotonin transporter knockout rat model of depression}

Investigating the role of specific genes, such as the SLC6A4 in humans has proven incredibly difficult, partly because of the strong genetic heterogeneity between individuals, even within the SLC6A4 gene itself, as mentioned above and partly because of the 
heterogeneity of depression itself. As a result, a direct link between a genetic variation in the SLC6A4 gene (or any other gene for that matter) and depression has so far not been established in studies in patients. Animal research, on the other hand, allows us to selectively manipulate one gene in a predictable manner, to investigate the causal relationship between that gene and behavioural changes.

A genetic alteration in the rat Slc6a4 gene was achieved by ENU-mutagenesis which induced a C to A transversion at position 3924 in the Slc6a4 gene resulting in a premature stop codon in the third exon (Homberg et al., 2007; Smits et al., 2006). This produced viable animals in which the Slc6a4 mRNA and functional protein are completely absent (referred to as SERT-/- rats) or reduced by about 50\% (SERT+/- rats; Homberg et al., 2007). The heterozygous (SERT+/-) animals demonstrate a similar loss-of-function in SERT activity as the human 5-HTTLPR $s$ allele and are therefore used as models for this condition (Kim et al., 2005; Lesch et al., 1996; Montañez, Owens, Gould, Murphy \& Daws et al., 2003).

In the homozygous (SERT-/-) rats the maximum rate of 5-HT reuptake is reduced by approximately $72.2 \%$ while for SERT $+/$ - rats this rate is reduced by $13.4 \%$. The less than $100 \%$ reduction in SERT-/- rats is thought to be due to residual uptake by the dopamine and/or noradrenaline transporter (Homberg et al., 2007). Reducing or eliminating SERT activity results in an increase in extracellular levels of 5-HT and basal levels of extracellular 5-HT increase nine-fold in the hippocampus of SERT -/- rats (Homberg et al., 2007).

The SERT KO rat is therefore suitable as a model for investigating the role of the Slc6a4 in depression and anxiety. SERT knockout mice also show similar depressive and anxious behaviours as humans (Holmes, Murphy \& Crawley, 2003). Similarly, behavioural testing revealed that SERT-/- rats spent less time in the centre of an open field and on the open arm of the elevated plus maze, had higher latencies before starting to feed during novelty suppressed feeding, had higher latencies to feed in a bright novel arena and had higher latency in emerging from their home cages, were less mobile in the forced swim test and consumed less sucrose compared to SERT+/+ rats (Kalueff, Olivier, Nonkes \& Homberg, 2010; Olivier et al., 2008). These behaviours are all indicative of greater anxiety and depression-like symptoms in the SERT-/- rats (Kalueff et al., 2010; Olivier et al., 2008). SERT -/- and SERT+/+ rats did not, however, differ in the number of immune-positive 5-HT neurons in the dorsal raphe nuclei (DRN), similarly, post-mortem studies of humans suffering 
from depression did not show reduced serotonergic neurons in the DRN (Olivier et al., 2008). Unfortunately, very little research has been done on SERT+/- rats so far. It has been questioned whether an absence of SERT leads to neurodevelopmental abnormalities and cortical disorganisation and whether this is an explanation for why genetic inactivation of the SERT induces anxiety and depression-like symptoms (Olivier et al., 2008).

\section{The Serotonin Paradox}

Putting all this research together, the role of serotonin presents us with a paradox. It has been shown that the most common medications given to treat depression and anxiety are selective serotonin reuptake inhibitors (SSRIs) that work by selectively inhibiting the serotonin transporter function thereby increasing extracellular levels of serotonin and alleviating the depressive or anxiolytic symptoms (Homberg et al., 2010). However, a genetic mutation of the SLC6A4 gene promoter region (5-HTTLPR) results in an allele ( $s$ allele) which similarly produces increased extracellular 5-HT levels through reduced SERT functioning (Heils et al., 1996) and is also linked to depressive and anxiolytic symptomology (Lesch et al., 1996; Furlong et al., 1998). Moreover, an increase in depression- and anxietylike behaviours and emotional problems in later life has been reported in SERT knockout rodent models and after early exposure to SSRIs (Kalueff et al., 2010; Olivier et al., 2008). Thus, a major shortcoming of the widely accepted monoamine hypothesis of depression is that it does not account for this serotonin paradox. See figure 1.

Figure 1

The serotonin paradox

Serotonergic differences
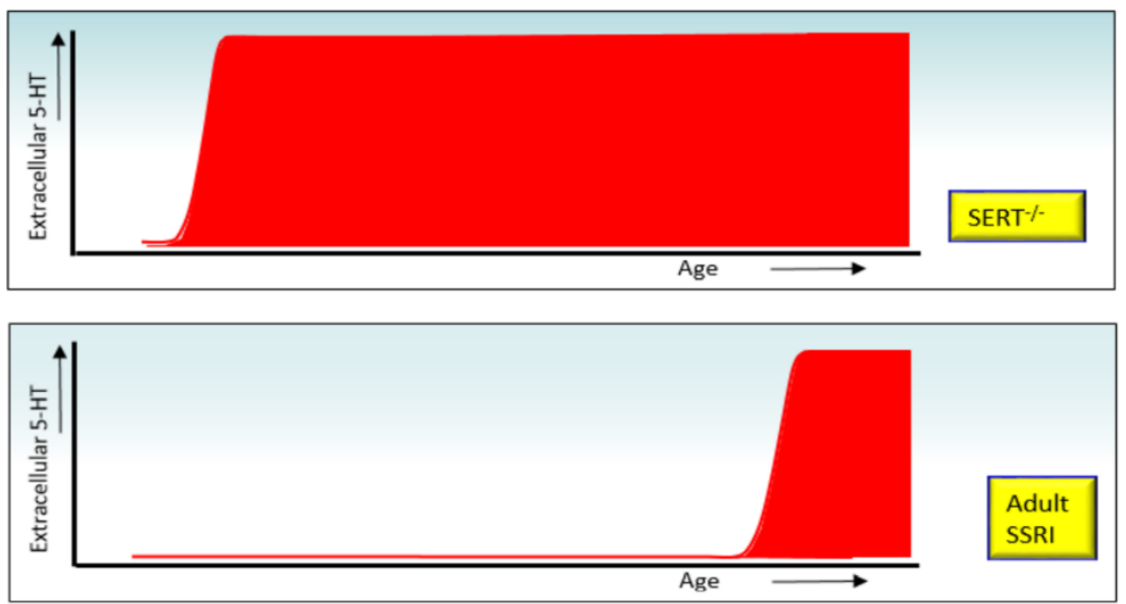

Psychological outcomes

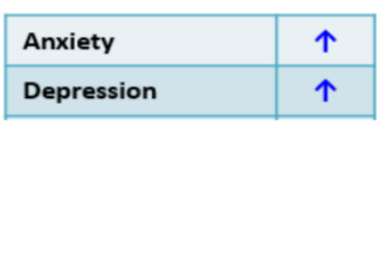

Anxiety

Depression $\downarrow$
$\downarrow$ 
Note. A visualisation showing the opposing serotonergic differences across lifetime and the corresponding anxiolytic and depressive risk outcomes between individuals lacking the serotonin trasnporter and adult SSRI use.

One apparent difference between these two scenarios is that extracellular 5-HT is chronically increased from early development onwards in $s$ allele carriers and SERT knockout models, while SSRIs only increase 5-HT in adulthood. This leads us to speculate that early exposure to increased 5-HT may affect brain development and result in depressive symptomology through altered neurocircuitry while individuals who develop depression in later life without this genetic background may have a reduction in their monoamines via mechanisms involving experience-induced changes to circuitry such as those associated with stress (Czéh et al., 2001; Djordjevic et al., 2012; Flugge, 1995; Manji, Drevets \& Charney, 2001; McKittrick, Blanchard, Blanchard, McEwen \& Sakai, 1995; McKittrick et al., 2000; Savitz, Lucki \& Drevets, 2009; Watanabe, Sakai, McEwen \& Mendelson, 1993). Indeed, it has been shown that depressed individuals do display altered neural connectivity (Greicius et al., 2007; Kaiser, Andrews-Hanna, Wager \& Pizzagalli, 2015; Perlman et al., 2012; Zeng et al., 2012).

While 5-HT is known to be a mood-regulating neurotransmitter in the adult brain, it initially has a crucial role in neurodevelopment (Azmitia, 2001; Gaspar, Cases \& Maroteaux, 2003). Ample evidence has shown that genetic and pharmacological disturbances in 5-HT signalling leads to the disruption of circuit formation, connectivity and altered cell morphology (Daubert \& Caudron, 2010; Gaspar et al., 2003; Homberg et al., 2010; Vitalis et al., 2007). In their review of animal and human studies of developmental exposure to increased 5-HT via SSRIs, Homberg et al. (2010) report long term effects on neurodevelopment which can be different to SSRI effects in adulthood e.g. structural and functional changes in the somatosensory cortex wiring. Thus, the early appearance of 5-HT and its receptors during foetal development, as well its effects on neurodevelopment, suggests that 5-HT acts as a developmental signal before it functions as a neurotransmitter. These findings suggest that we could consider depression a problem of neuronal circuitry disturbances due to altered monoamine levels. 
5-HT is required for normal embryonic development (Buznikov, Lambert \& Lauder, 2001; Côté et al., 2007; Whitaker-Azmitia, Druse, Walker \& Lauder, 1995). 5-HT is one of the key regulators of neurogenesis, differentiation, survival, and neuronal migration, dendrite formation, synaptogenesis, axon branching, morphogenesis, apoptosis and is released from developing neurons before synapses are even formed (Azmitia, 2001; Brezun \& Daszuta, 1999; 2000a, 2000b; Hansson, Mezey \& Hoffman, 1999; Lauder, 1990; Levin, Buznikov \& Lauder, 2006; Matsukawa, Nakadate, Ishihara \& Okado, 2003; Vitalis et al., 2007; Vitalis \& Parnavelas, 2003).

The serotonergic regulation of all these neurodevelopmental processes suggest that disturbances in early 5-HT levels will alter the connectivity of neural circuitry and effectively alter neuroplasticity. Such neurodevelopmental changes could be underlying emotional and behavioral impairments seen in $s$ allele carriers of the 5-HTTLPR and in SERT knockout models. Humans, for example, undergo a period of high brain 5-HT synthesis during early childhood and disturbances in which are linked to neurological disorders such as autism (Chugani, 2002). SERT knock out rats also display altered raphe-prefrontal network formation compared with wild-types due to altered chemotrophic interactions between origin and targets of the 5-HT projection system (Witteveen et al., 2013).

The serotonergic system is an early forming component of the CNS (Aitken \& Törk, 1988; Lauder \& Bloom, 1974; Lauder, 1990; Olson \& Seiger, 1972). Serotonergic neurons are seen starting to develop in the foetal rat brain at embryonic day 12-13 and 5-HT fibre outgrowth begins at E13 (Aitken \& Törk, 1988; Hansson et al., 1999; Lauder \& Bloom, 1974; Lauder, 1990; Lidov \& Molliver, 1982; Olson \& Seiger, 1972; Wallace \& Lauder, 1983). In humans, 5-HT is detected as early as the 7th week of gestation (Olson et al., 1973). Moreover, 5-HT is not only involved in serotonergic system development but also in most non-serotonergic circuits (D'Amato et al., 1987; Daubert and Condron, 2010; Hansson et al., 1999; Lauder \& Krebs, 1978; Lidov \& Molliver, 1982; Sikich, Hickok \& Todd, 1990; Vitalis \& Parnavelas, 2003; Vitalis et al., 2007). Transient 5-HT expression is seen throughout entire brain/ in most brain structures including in non-serotonergic neuronal cells as early as E12 and occurs through uptake into these cells by plasma membrane transporters rather than synthesis (Cases et al., 1998; De Vitry et al., 1986). 5-HT also initiates and amplifies its own synthesis in embryonic cells (De Vitry et al., 1986). 
SERT expression begins at E10-E12 in rodents, preceding synapse formation (Brüning, Liangos \& Baumgarten, 1997; Hansson et al., 1999; Zhou, Sari \& Zhang, 2000). The transient presence of Slc6a4 mRNA in the sensory processing, affect (limbic system), neuroendocrine (hypothalamus and pituitary) and circadian rhythm systems during embryonic development also indicates a role for 5-HT during development on cells which respond, or will respond, to 5-HT in adulthood (Hansson et al., 1999; Lebrand et al., 1998). In mice, SERT is first expressed in serotonergic Raphe neurons at mid gestation (E11) then rapidly appears in non-serotonergic neurons and ends in the non-serotonergic neurons during the second postnatal week, which coincides with the maturation of these neural circuits (Homberg et al., 2010). These studies suggest that alterations in (early) 5-HT levels may have far reaching consequences, not just for the serotonergic system but beyond that for other neurotransmitters as well.

Maternal 5-HT drives embryonic morphogenesis before the appearance of foetal serotonergic neurons and foetal tryptophan hydroxylase expression (Côté et al., 2007). This suggests that (genetic) changes in maternal 5-HT can have long-lasting consequences for the development of the offspring. Inhibition of embryonic 5-HT synthesis leads to a delayed onset of differentiation of 5-HT target cells (Lauder, 1990; Lauder \& Krebs, 1978).

Axonal development overlaps with serotonergic neuron development (Lidov \& Molliver, 1982; Seiger \& Olson, 1973). SERT is expressed in axons, dendrites and growth tips of embryonic neurons including transient expression in non-serotonergic neurons and 5HT regulates axon guidance cues (e.g., Netrin-1; Bonnin et al., 2007; Narboux-Nême et al., 2008; Zhou et al., 2000). Both high and low levels of 5-HT affect developmental axon growth. For example, 5-HT depletion results in a decrease in the number and density of axons in both high- and low-density serotonergic axon regions of neonatal rat somatosensory cortices (D'Amato et al., 1987), while SERT and Monoamine oxidase A inhibition produces abnormal segregation of axons in the retinogeniculate and somatosensory thalamocortical systems (Salichon et al., 2001; Upton et al., 2002). Similarly, a broad acting 5-HT receptor agonist 5-methoxytryptamine (5-MT) had a biphasic effect on serotonergic neuron terminal growth, where at low doses it was inhibitory but at high doses it stimulated terminal growth (Shemer, Azmitia \& Whitaker-Azmitia, 1991; Whitaker-Azmitia, Shemer, Caruso, Molino \& Azmitia, 1990b). 5-HT1A agonist treatment also prevents the loss of dendritic spines that typically follows neonatal 5-HT depletion and increases dendritic tree length and branching 
of cholinergic neurons, while 5-HT1A antagonist treatment results in dendritic spine loss comparable to that of the 5-HT depletion (Riad et al., 1994; Yan, Wilson \& Haring, 1997b).

5-HT depletion leads to a decrease in the neurotrophic growth factor S100 $\beta$ (which typically stimulates neurite growth; Azmitia et al., 1990; Haring et al., 1993) and decreases dendritic spine density, neurite extension and synapse formation in dentate gyrus (DG) granule cells (Brezun \& Daszuta, 1999; Mazer et al., 1997; Yan et al., 1997a). It also reduces Microtubule-associated protein 2 (MAP-2) immunoreactivity density, which has a role in dendritogenesis and is an immunocytochemical marker for dendrites (Azmitia, Rubinstein, Strafaci, Rios \& Whitaker-Azmitia, 1995; Mazer et al., 1997). The converse also occurs with SSRI fluoxetine administration leading to increases in S100ß (Haring et al., 1993).

Astrocytes are central to plasticity within the brain, since they not only make glucose available to neurons, but also provide adhesion and trophic factors for neuronal growth and migration (Azmitia, 2001). Astrocytes also express 5-HT1A receptors and 5-HT1A agonist stimulation leads to the release of $S 100 \beta$ and maturation of astrocyte morphology (Azmitia, 2001; Azmitia et al., 1995; Whitaker-Azmitia, Murphy, Azmitia \& 1990a). Moreover, S100ß is transiently observed in the developing foetal raphe region suggesting a functional interaction between astrocytes and neurons during development which facilitates maturation of these cell types via the astrocytic 5-HT1A receptor (Whitaker-Azmitia et al., 1990a).

Synapses are a crucial substrate for plasticity in adult neural circuits (Holtmaat \& Svoboda, 2009; Trachtenberg et al., 2002). The timing of serotonergic innervation coincides with a period of pronounced and critical synpatogenesis (D'Amato et al., 1987).

Synaptogenesis of neurons in the rat begins on E18-19 in the medial raphe nuclei and is first observed at the perikarya of these neurons at E20 (Lauder \& Bloom, 1975). In humans, the earliest synaptic connections form by the 5th gestational week with intense synaptogenesis occurring during the first few years of life, particularly during the first year (Tau \& Peterson, 2010). The 28th gestational week in human pregnancy is a period of intense synaptogenesis and corresponds to postnatal day (PND) 7-8 in rats (Liu, Chow \& Sherry, 2013a; Oberlander et al., 2009; Tau \& Peterson, 2010; Uytun, 2018). Timelines depicted in figures 2 and 3.

Figure 2

Time line of human neurodevelopmental events 


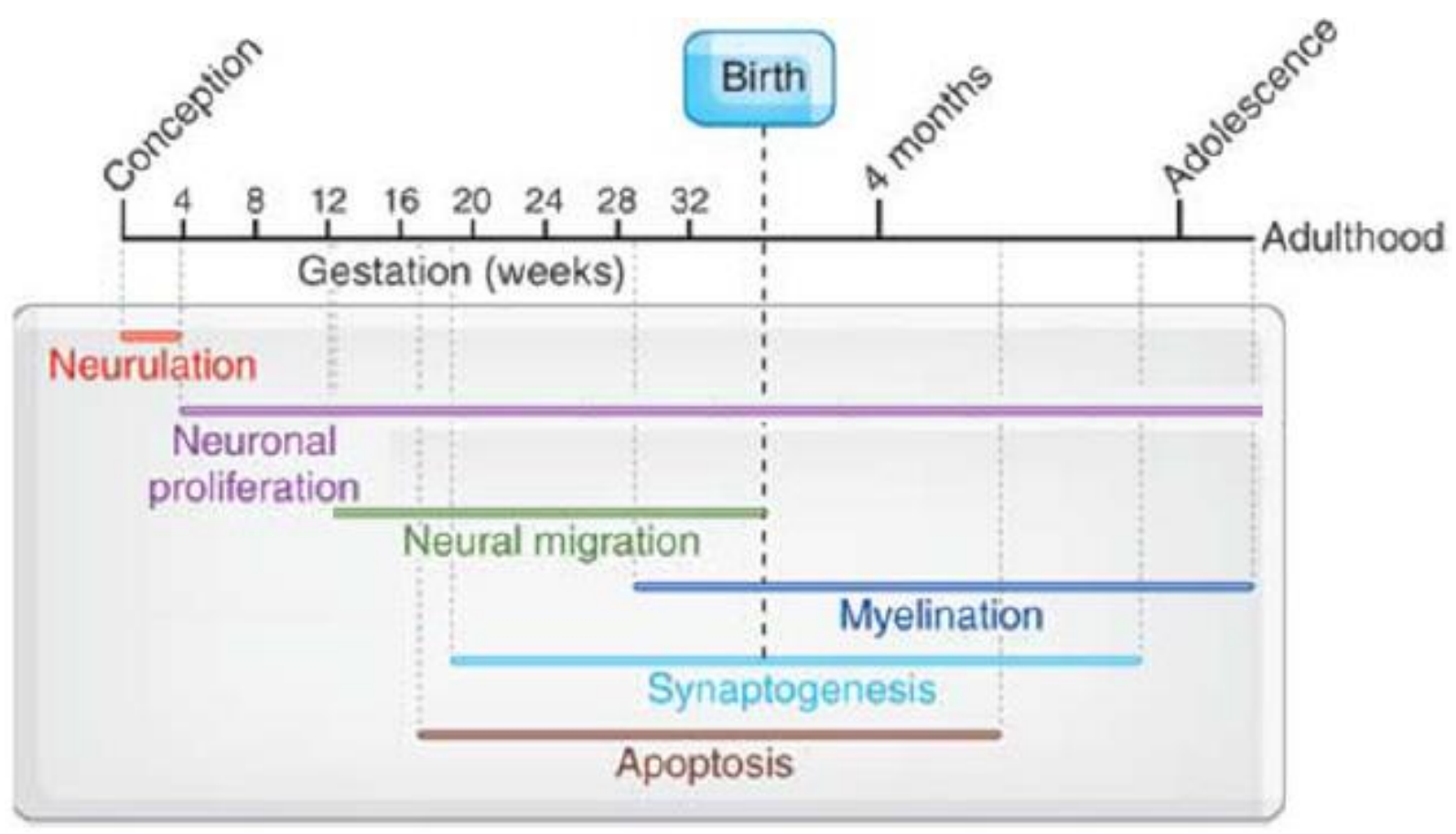

Note. Modified from Tau and Peterson (2010)

Figure 3

Comparison of human and rat developmental timelines

Gestational weeks for human
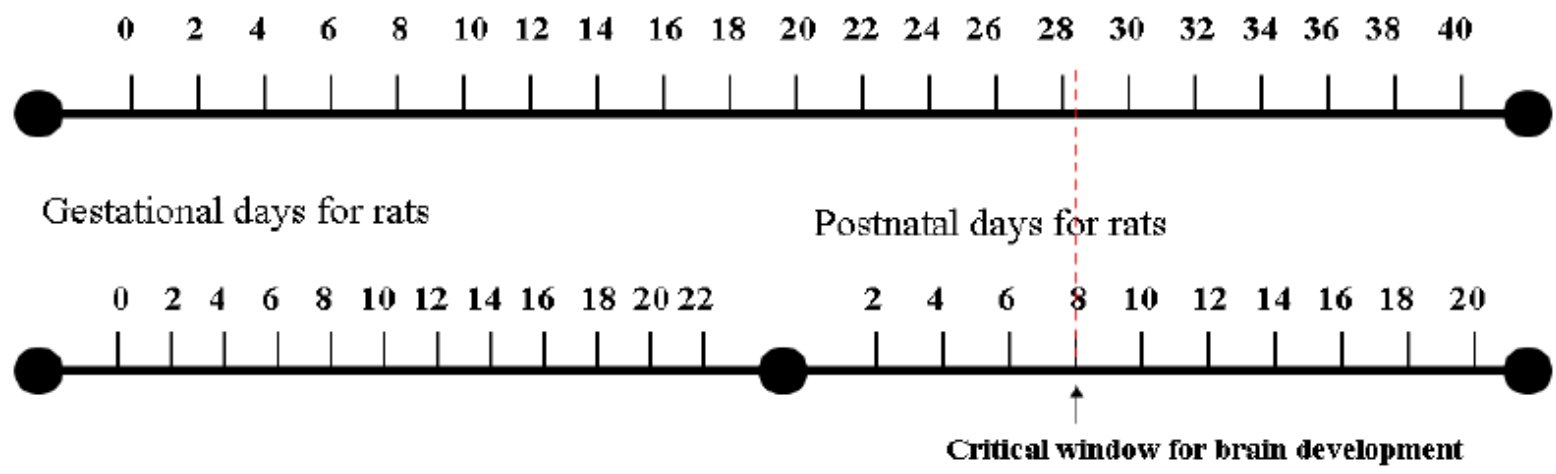

Note. Adapted from Liu et al. (2013a)

5-HT depletion and 5-HT1A antagonism both decrease dendritic spines, and this is accompanied by a decrease in synaptic density (Faber \& Haring, 1999). Similarly, new research from our own laboratory found that neurons in primary cell cultures showed a gene dosage dependent decrease in spine density, with homozygous SERT knockouts having the smallest, and WT, the largest number of spines (Chaji, Venkatesh, Shirao, Day \& Ellenbroek, 2021). On the other hand, antidepressant treatment increases synapses in the hippocampal 
CA1 region (Chen, Madsen, Wegener \& Nyengaard, 2010). Activation of the 5-HT7 receptor promotes neurite elongation, dendritic spine formation and synaptogenesis while deactivation decreases the number of spines (Speranza et al., 2017). 5-HT2A receptors also have a role in synaptogenesis. For example, Niitsu, Hatnada, Hamaguchi, Mikuni and Okado (1995) demonstrated that treatment of chick embryos with a 5-HT2A receptor agonist (DOI) and antagonist (ketanserin) increase and decrease synaptic density in the lateral motor column of the spinal cord, respectively.

5-HT clearly plays a role in regulating neuronal connectivity. Overall, the expression of 5-HT prior to synaptogenesis and in growing serotonergic fibre terminals as well as the appearance of 5-HT receptors in cells prior to 5-HT synthesis suggests a role for 5-HT in directing this growth and in effect, the connectivity of 5-HT and several other neuronal systems (Aitken \& Törk, 1988; Côté et al., 2007; Lauder, 1990). Importantly, disturbances in 5-HT homeostasis alter neuronal connectivity.

\section{Neuroplasticity/neural circuitry}

In addition to its role in development, 5-HT also shapes the brain networks and connectivity underlying a wide range of essential neuronal functions ranging from perception and cognitive appraisal to emotional responses in the mature brain (Hansson et al., 1999; Lebrand et al., 1998). Neuroplasticity refers to the adaptive changes in neuronal function which allow the brain to learn from experiences and sensory input (Feldman, 2009). This includes structural plasticity (i.e., changes in spine and dendrite morphology and wiring and neurogenesis) and Hebbian synaptic plasticity (i.e., functional changes in synapse signalling strength) which are both affected by 5-HT (Feldman, 2009; Holtmaat \& Svoboda, 2009; Krishnan \& Nestler, 2008; Pittenger \& Duman, 2008; Teissier, Soiza-Reilly \& Gaspar, 2017).

Activity at synapses is an important contributor to synaptic plasticity through different patterns of receptor activation or second messenger regulation of synaptic vesicle release machinery and can lead to long term potentiation (LTP) or long term depresson (LTD) (Leenders \& Sheng, 2005; Lüscher, Nicool, Malenka \& Muller, 2000; Sheng \& Kim, 2002; Stepan, Dine \& Eder, 2015). LTP represents the synaptic plasticity involved in learning and memory and is affected by 5-HT (Nguyen \& Kandel, 1996; Pittenger \& Duman, 2008; Saxe et al., 2006). 
Disturbances in 5-HT-moderated synaptic signalling alters synaptic plasticity and neurocircuitry (Lesch \& Waider, 2012; Niitsu et al., 1995). For example, behavioural testing of 5-HT depleted animals showed delayed extinction of behaviour and deficits in spatial learning and memory systems located predominantly within the hippocampus and are consistent with the altered synaptic morphology observed (Mazer et al., 1997). SERT ablation as well as SSRI treatment also increase prefrontal cortex glutamate synapses on 5HT and GABA neurons in the DRN (Soiza-Reilly et al., 2019). The 5-HTTLPR is also associated with amygdala activation and $s$ allele carriers show greater coupling between the amygdala and the ventromedial prefrontal cortex, this may contribute to the abnormally high activity in the amygdala and medial prefrontal cortex seen in major depression (Hariri et al., 2002; Heinz et al., 2004; Munafò et al., 2008). 5-HT also influences the postnatal remodelling of circuitry (Teissier et al., 2017).

\section{Neurogenesis}

Typically, neurogenesis (the formation of new neurons) occurs from prenatal development into adulthood (Eriksson et al., 1998; Jacobs, van Praag \& Gage, 2000). In mammalian brains, most neurons are created during the pre- and perinatal period of development (Jacobs et al., 2000).

A common notion about the function of adulthood neurogenesis is that new neurons in the adult brain are beneficial as they increase neuroplasticity. However, the amount of adult neurogenesis decreases with increasing brain complexity. While some lower vertebrates can regenerate entire brain parts, mammalian adult neurogenesis is limited to the subventricular zone (SVZ) of the lateral ventricles which generate the granule cells that end up in the olfactory bulb and the subgranular zone (SGZ) of the dentate gyrus (at the border between the hilus and granule cell layer) which gives rise to granule cells from neural stem/progenitor cells in the hippocampus (Altman \& Das, 1966; Alvarez-Buylla \& GarcíaVerdugo, 2002; Duan, Kang, Liu, Ming \& Song, 2008; Duman, 2004; Ehninger \& Kempermann, 2008; Eriksson et al., 1998; Gage, 2002; Kempermann, Wiskott \& Gage, 2004a, Kempermann, Jessberger \& Kronenberg, 2004b; Kuhn, Dickinson-Anson \& Gage, 1996; Vadodaria \& Jessberger, 2014; van Praag et al., 2002; Zaverucha-do-Valle et al., 2013; Zhao, Deng \& Gage, 2008). Depicted in figure 4. This restricted neurogenesis is suggestive of a trade-off between the benefits of producing new neurons and the complications they 
create for the network structure into which they need to integrate (Kempermann et al., 2004a).

These neurogenic niches are very important as their unique microenvironments and constituent regulatory environmental cues allow neurogenesis to occur, even transplanting neural stem/ progenitor cells from non-neurogenic brain regions allows them to differentiate into neurons in these zones (Ehninger \& Kempermann, 2008; Shihabuddin, Horner, Ray \& Gage, 2000; Suhonen, Peterson, Ray \& Gage, 1996). In these two neurogenic regions the benefits of neurogenesis outweigh the complications and therefore, must serve an important hippocampal function (Kempermann et al., 2004a).

Hippocampal neurogenesis is the process of neural stem cells/ progenitors in the SGZ dividing and maturing into functional neurons (Ming \& Song, 2011). This occurs through radial-glia-like stem cells (type-1 cell) with astrocytic properties progressing through at least two stages of amplifying lineage-determined progenitor cells (type- 2 and type- 3 cells) to early postmitotic and to mature neurons (See figure 4; Eisch et al., 2008; Kempermann et al., 2004b). Granule cell formation occurs during pregnancy and continues postnatally (Bayer, 1980; Seress, Ábrahám, Tornóczky \& Kosztolányi, 2001). One estimate posits that up to 9000 new cells are generated daily in the adult rat brain (Cameron \& Mckay, 2001).

Figure 4.

The process of neurogenesis

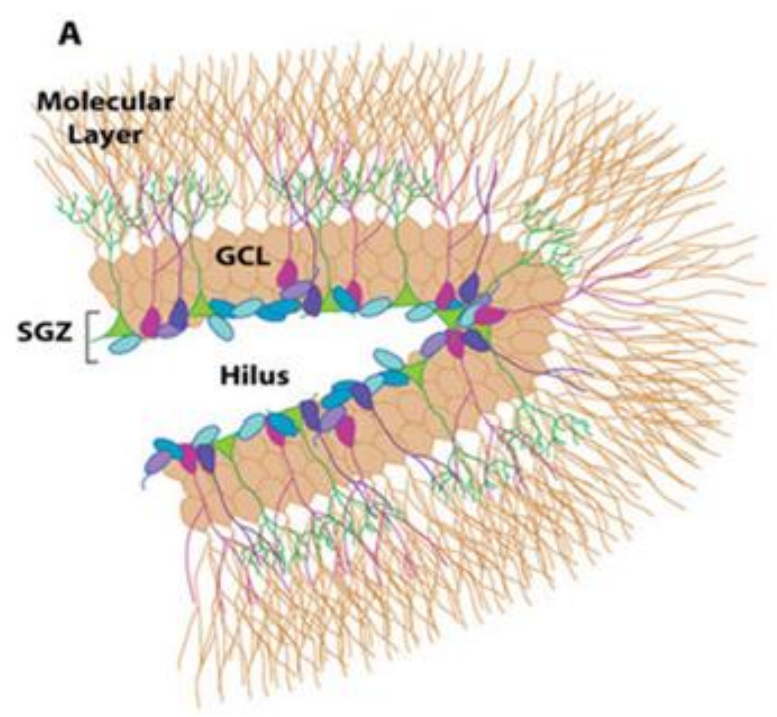

B

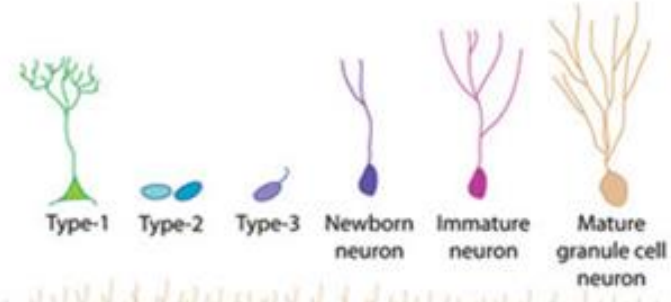

C

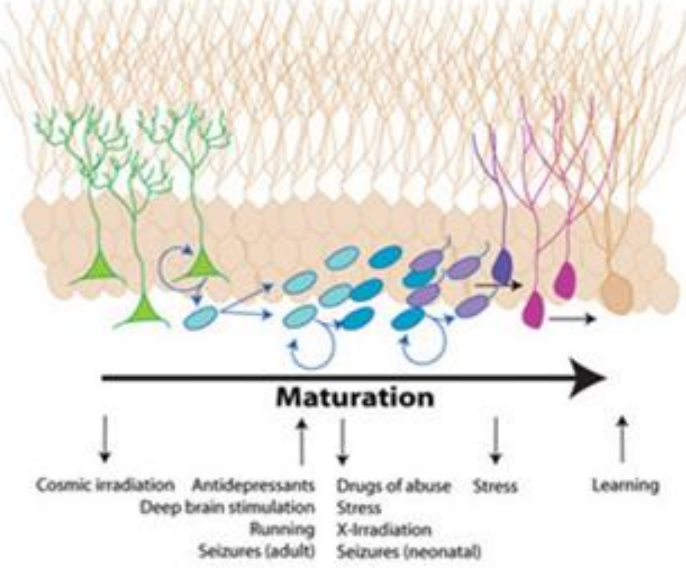


Note. Dentate gyrus layers depicted in A. B and C illustrate the progression through neurogenesis stages. $\mathrm{C}$ also notes factors effecting the maturation of cells undergoing neurogenesis. From Eisch et al. (2008).

The majority of new cells (50-80\%) in the DG differentiate into neurons while some differentiate into glial cells (Barlow \& Targum, 2007; Warner-Schmidt \& Duman, 2006). Once in the granule cell layer, cells that will become neurons, develop the characteristics of adult granule cells which includes dendrite extension into the molecular layer, axon elongation into the CA3 pyramidal cell layer via the mossy fibre pathway where they terminate in synapse and interneuron rich areas, receiving synaptic inputs, exhibit the classic plasticity period of developmentally generated neurons (Ge, Yang, Hsu, Ming \& Song, 2007) and activity-dependant gene expression (Duman, 2004; Ehninger \& Kempermann, 2008; Jessberger \& Kempermann, 2003; Kempermann et al., 2004b; van Praag et al., 2002).

Early postnatal hippocampal development is a crucial period of neurogenesis (Arnold \& Trojanowski, 1996; Seress et al., 2001). At birth, the dentate gyrus only contains approximately $70 \%$ of the number of cells seen in adulthood, the remaining $30 \%$ are produced postnatally (Bauer, 2004). In humans, major cortical neurogenesis takes place between gestational weeks 6-16 with further cortical development continuing postnatally (Sidman \& Rakic, 1973) while several early cellular events characterising cortical embryonic/foetal neurogenesis occur by 6-7 weeks (Choi, 1988). It is only after 12-15 postnatal months that hippocampal morphology resembles the adult structure (Bauer, 2004). Studies of infant memory indicate that this early-developing hippocampus is largely responsible for early competencies (Bauer, 2004; Gómez \& Edgin, 2016).

The adult brain faces a challenge in that it must not only maintain stability in neural circuitry, but also allow plasticity to be able to change and adapt to environmental challenges. Neural circuits are undeniably shaped by the environment and thus change across the lifetime (Abrous, Koehl \& Le Moal, 2005; Teissier et al., 2017). Experience and learning in early critical periods, as well as in adulthood, underlie sensory plasticity and involve synaptic regulation including formation, removal and morphological remodelling of synapses and dendritic spines (Feldman, 2009; Holtmaat \& Svoboda, 2009; Teissier et al., 2017; Trachtenberg et al., 2002; Wilbrecht, Holtmaat, Wright, Fox \& Svoboda, 2010). 
Environmental stimulion is a key driver of neuroplasticity and learning (Novkovic, Mittmann \& Manahan-Vaughan, 2015). Environemental enrichment is an animal model for the environmental stimuli-based neural plasticity and circuitry modulation seen in normal brain functioning and is shown to improve LTP (Novkovic et al., 2015). Similarly, activitydependant neurogenesis occurs when excitatory stimuli act directly on neural progenitor cells through excitatory receptors to stimulate new neuron production (Deisseroth et al., 2004; Lüscher et al., 2000).

There is emerging consensus that these newborn neurons are involved in learning and memory (Bath, Akins \& Lee, 2012; Gould, Beylin, Tanapat, Reeves \& Shors, 1999). Manipulations that increase adulthood neurogenesis (AHN), such as environmental enrichment, exercise and antidepressant treatment have been found to enhance performance in pattern separation, verbal declarative memory, spatial learning and LTP (Karabeg et al., 2013; Olson, Eadie, Ernst \& Christie, 2006; Sahay et al., 2011; van Praag, Christie, Sejnowski \& Gage, 1999; Vermetten, Vythilingam, Southwick, Charney \& Bremner, 2003). On the other hand manipulations that decrease AHN, such as aging (Drapeau et al., 2003) or ablation of neurogenesis, impair performance in learning and memory such as fear conditioning (Shors et al., 2002; Winocur et al., 2006), spatial navigation learning (Drapeau et al., 2003; Hu et al., 2009; Imayoshi et al., 2008; Mohapel, Leanza, Kokaia \& Lindvall, 2005; Morris et al., 1982; Zhang et al., 2008), spatial pattern separation (Clelland et al., 2009; Kim, Christian, Ming \& Song, 2012), impair normal endocrine responses and increase behavioural stress responses (Snyder, Soumier, Brewer, Pickel \& Cameron, 2011). Moreover, hippocampal lesion-induced memory impairments illustrate that neurogenesis is involved in memory processes (Shors et al., 2001, 2002; Imayoshi et al., 2008; Vargha-Khadem et al., 1997).

However, different AHN-reducing manipulations have produced conflicting results (Saxe et al., 2006) with some affecting spatial learning but not fear conditioning (Zhang et al., 2008) or vice versa (Shors et al., 2002). This is possibly due to the functional differences observed in the septotemporal axis, as lesion studies demonstrate that the septal pole is more involved in spatial learning and memory while the temporal pole plays a greater role in emotional behaviours (Fanselow \& Dong, 2011; Kjelstrup et al., 2002; Moser, Moser, Forrest, Anderson \& Morris, 1995; Moser \& Moser, 1998; Sahay \& Hen, 2007; Tanti \& Belzung, 2013). 


\section{The hippocampus}

The hippocampus is critical to the process of forming and recovering certain types of memory (Eichenbaum, 2010; Squire, Stark \& Clark, 2004). It is a bilateral subcortical structure in the temporal lobe and has a laminar (layered) organization (Andersen, Soleng \& Raastad, 2000; Sapolsky, 2000; Sloviter \& Lømo, 2012).

The hippocampal formation consists of the hippocampal proper which is divided into four zones, called 'cornu ammonis' 1-4 (CA1 - CA4) and its related parts- the dentate gyrus and subiculum (Giap et al., 2000; Mendoza \& Foundas, 2008). CA1 is the outermost segment merging with the subiculum while CA3 and CA4 (sometimes referred to as CA3 together) is the innermost segment of the hippocampus proper adjacent to the dentate gyrus (Mendoza \& Foundas, 2008; See figure 5).

Figure 5.

Saggital section of rat hippocampus on PND60.

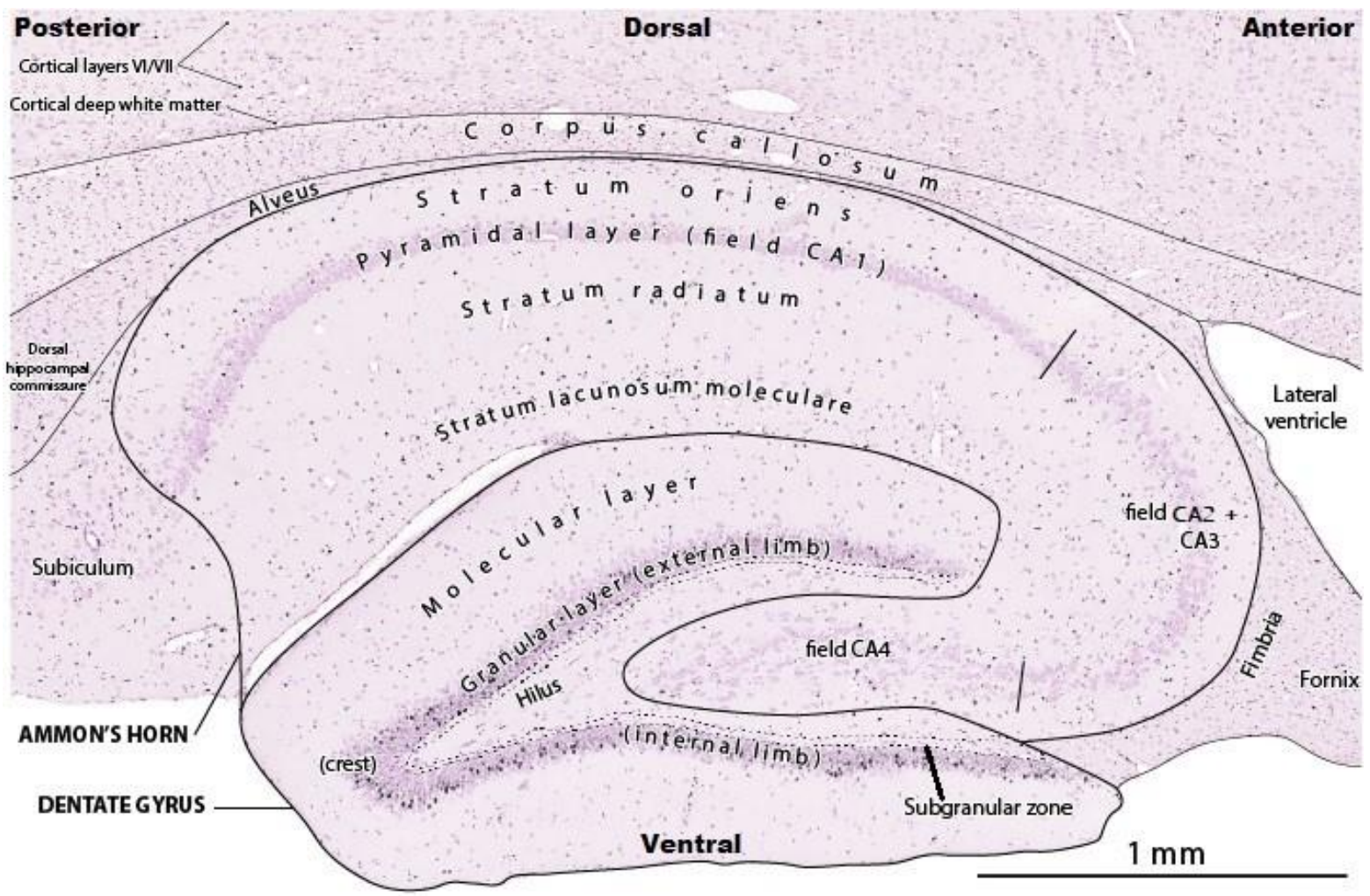

Note. Modified from Brain Developmental Maps (2020).

The hippocampus proper is comprised of three distinct layers. The pyramidal cell layer (stratum pyramidal) contains pyramidal cell bodies- the principal cells of the 
hippocampus. The molecular layer (divided into a more external stratum lacunosum moleculare and a more internal stratum radiatum) consists of apical dendrites from pyramidal cells and axons originating from various other regions. Lastly, the polymorphic layer (stratum oriens) contains various interneurons, including multipolar GABAergic basket cells (Giap et al., 2000; Jacobson \& Marcus, 2008).

The dentate gyrus also consists of three layers (see figure 4). The molecular layer contains the dendrites of granule cells. The granule cell layer of small unipolar neurons whose dendrites emerge from the apical end of the cell and into the molecular layer. Lastly, there is a polymorphic cell layer (aka the hilus) which is continuous with the CA4 (Amaral et al., 2007; Giap et al., 2000; Jacobson \& Marcus, 2008).

The subiculum and hippocampus are mainly comprised of efferent pyramidal neuronal cells, which project to several structures, while the dentate gyrus sends projections to the hippocampus alone (Giap et al., 2000). The pyramidal and granule cells are excitatory neurons utilizing the neurotransmitter glutamate, while basket cells in the polymorphic layers are inhibitory GABA-ergic interneurons (Giap et al., 2000; Jacobson \& Marcus, 2008).

The trisynaptic circuit is a relay of hippocampal synaptic transmission beginning with projections from the entorhinal cortex that synapse on the DG via granule cell fibres (the perforant path), the DG then synapses on CA3 pyramidal cells via their mossy fibres, the CA3 then synapse in the CA1 via Schaffer collaterals (Insausti \& Amaral, 2003; Sapolsky, 2000).

The septotemporal axis of the hippocampus influences the rate of neurogenesis as studies have found that for example the septal (dorsal) DG has a faster rate of neurogenesis than the temporal (ventral) DG, and these new neurons mature faster in the septal DG than the temporal DG (Piatti et al., 2011; Snyder, Ferrante \& Cameron, 2012). Snyder et al. (2012) found different rates of neuronal maturation along the septotemporal and transverse axis of the DG and posit that the prolonged maturation in the temporal region translates to a longer duration of plasticity in this region.

5-HT is an important component of hippocampal function. The dentate gyrus receives plenty of serotonergic innervation and is enriched with 5-HT1A receptors (Azmitia et al., 
1996; Azmitia \& Segal, 1978; Blier \& Ward, 2003; Freund, Gulyás, Acsády, Görcs \& Tóth, 1990; Gérard et al., 1994; Klempin et al., 2010). Serotonergic fibres project diffusely throughout the brain and into the dentate gyrus of the hippocampus where they form synapses with granule cells and interneurons (Alenina \& Klempin, 2015). 5-HT is implicated in cell proliferation as raphe lesioning and 5-HT depletion leads to decreased granule cell proliferation in the dentate gyrus, while serotonergic raphe grafts reversed these post-lesion decreases in granule cell immunostaining and decreases the amount of the neural cell adhesion molecule PSA-NCAM which is a marker for molecular plasticity in the DG (Alvarez-Buylla \& García-Verdugo, 2002; Brezun \& Daszuta, 1999, 2000a; Djordjevic et al., 2012). These effects are reversed after partial 5-HT hippocampal reinnervation (Brezun \& Daszuta, 2000b).

Considerable evidence implicates the serotonergic system in the stimulation of neurogenesis. Firstly, conditions associated with decreased neurogenesis such as aging (Kempermann, Kuhn \& Gage, 1998; Kuhn et al., 1996), high corticosterone levels (Campbell \& MacQueen, 2004), stress (Mirescu \& Gould, 2006) and malnutrition (Debassio, Kemper, Tonkiss \& Galler, 1996) also affect the serotonergic system by decreasing 5-HT fibre or 5HT receptor density or decreasing extracellular 5-HT levels for example (Blatt, Jin-Chung, Rosene, Volicer \& Galler, 1994; Chalmers, Kwak, Mansour, Akil \& Watson, 1993; Gould, 1999; McKittrick et al., 1995; Míguez, Aldegunde, Paz-Valiñas, Recio \& Sánchez-Barceló, 1999; Neumaier, Petty, Kramer, Szot \& Hamblin, 1997; Watanabe et al., 1994). Conversely, conditions associated with increased neurogenesis such as exercise, environmental enrichment, BDNF and antidepressant treatment also increase 5-HT signalling (Dekeyne et al., 2008; Duman, 2004a; Kim et al., 2012; Klempin et al., 2013).

Studies show that conditions associated with exacerbating depression, such as stress (Pittenger \& Duman, 2008) suppress AHN (Dranovsky \& Hen, 2006; Mirescu \& Gould, 2006; Nasir \& Khan, 2011; Perera et al., 2007; Schoenfeld \& Gould, 2012), while those that alleviate depressive symptomology such as exercise (Cooney et al., 2013; Liu et al., 2013b; Zheng et al., 2006), increase AHN (Olson et al., 2006). Moreover, several stress models of depression, such as learned helplessness and chronic mild stress, are associated with reductions in hippocampal neurogenesis (Chen et al., 2010; Lee et al., 2006; Vollmayr, Simonis, Weber, Gass \& Henn, 2003). Stress-induced reduction in neurogenesis is also a causal factor in precipitating episodes of depression (Jacobs et al., 2000). 
Neuroanatomical changes are also associated with depression and mood disorders. For example, studies consistently show that individuals with depression have lower hippocampal volumes (usually a larger reduction in the right hemisphere), including less granule cells, than healthy controls (Boldrini et al, 2013; Bremner et al., 2000; Campbell, Marriott, Nahmias \& MacQueen, 2004; Kronmüller et al., 2009; Sheline ,Wang, Gado, Csernansky \& Vannier, 1996; Videbech \& Ravnkilde, 2004) with longer illness duration directly relating to the magnitude of hippocampal decrease (MacQueen et al., 2003; McKinnon, Yucel, Nazarov \& MacQueen, 2009; Sheline, Sanghavi, Mintun \& Gado, 1999; Stockmeier et al., 2004). The fact that hippocampal volume differences occur in depressed children lends further support to the idea that the early postnatal period is an indeed important period for hippocampal neurogenesis and that its dysregulation is associated with depression (McKinnon et al., 2009).

Depressed individuals also show impairments in hippocampal dependant functions such as impaired learning, memory, particularly including impaired performance on hippocampal dependant memory tests (Austin, Mitchell \& Goodwin, 2001; Bremner, Vythilingam, Vermetten \& Vaccarino \& Charney, 2004; Burt, Zember \& Niederehe, 1995; Ilsley, Moffoot \& O’Carroll, 1995; MacQueen et al., 2003). Individuals that scored high on the Beck Depression Inventory show impairment in the hippocampus-dependant delayed match to sample task (Becker, MacQueen \& Wojtowicz, 2009).

Neurogenesis is also regulated by antidepressant treatment and appears to be important for the therapeutic effects of antidepressants (Airan et al., 2007; Encinas, Vaahtokari \& Enikolopov, 2006; Sahay \& Hen, 2007; Santarelli et al., 2003; Surget et al., 2008).

While antidepressants have relatively rapid effects on monamine signalling, there is typically a latency of more than 3 weeks of treatment before they are clinically efficacious (Sapolsky, 2004; Wong \& Licinio, 2001) suggesting that they act through slow neurochemical and structural changes, such as neurogenesis, within the brain (Duman, 2004; Duman \& Monteggia, 2006; Massart et al., 2012; Santarelli et al., 2003). Antidepressant treatment upregulates hippocampal neurogenesis, both in the presence and absence of depressive symptomology/behaviours (Airan et al., 2007; Dranovsky \& Hen, 2006; Duman, 
2004; Encinas et al., 2006; Klempin et al., 2010; Malberg et al., 2000; Perera et al., 2007; Santarelli et al., 2003; Warner-Schmidt \& Duman, 2006). Moreover, the beneficial effects of antidepressants are dependent on neurogenesis (Airan et al., 2007; Santarelli et al., 2003). These findings are in line with the studies mentioned above, that neurogenesis may be dysregulated in depression (Duman, 2004a; Manji et al., 2001).

\section{Summary}

This mounting evidence implicates neurogenesis in the neurobiological pathology of depression (Paizanis, Hamon \& Lanfumey, 2007). Evidence, particularly from studies of antidepressant effects on neurogenesis, suggests that it is partially through neurogenesis that antidepressants elicit their beneficial effects (Santarelli et al., 2003; Surget et al., 2008). Moreover, it appears that 5-HT is a potent regulator of the hippocampal neurogenesis where increased 5-HT appears to be conducive to increased neurogenesis (Banasr, Hery, Printemps \& Daszuta, 2004; Klempin et al., 2013). What remains to be examined are the effects of genetically increased 5-HT levels and neurodevelopmental changes that could underlie depression.

Given that 5-HT has such a crucial role in embryonic brain development, the role of the maternal influence over these processes needs to be determined. The placenta is the primary source of 5-HT for the developing feotal brain (Côté et al., 2007; Huang, Zhang, Di \& Zhang, 1998; Kliman et al., 2018; Rosenfeld, 2020). Alterations in maternal 5-HT produce long term morphological brain changes in foetal rats (Côté et al., 2007; Matsukawa et al., 2003; Vitalis et al., 2007) and leads to maladaptive depressive- and anxiety-like behavioural phenotypes in offspring (Kesper \& Homberg, 2015; Shah, Courttiol, Castellanos \& Teixeira, 2018). The maternal SERT genotype impacts the placental 5-HT levels in the foetal forebrain, thus effecting foetal neurodevelopment (Muller et al., 2017). For example, the maternal SERT Ala56 genotype is associated with decreased placental and embryonic forebrain 5-HT levels (Muller et al., 2017).

As 5-HT participates in so many crucial neurodevelopmental processes, it is important to examine more global changes in SERT knockout neurochemistry. Matrix assisted laser desorption ionization imaging mass spectrometry (MALDI- IMS) is a valuable technique which allows for the detection, identification and distribution mapping of chemical compounds within tissue (Schubert, Weiland, Baune \& Hoffmann, 2016). Thus, further 
analysis of not only 5-HT but other neurotransmitter, receptor, and metabolite changes can be accomplished which would allow us to draw conclusions on larger-scale pathways involved in depression.

\section{This study}

The monoamine hypothesis of depression proposes that because antidepressant alleviate depression, it must arise from a lack of monoamines, however it does not account for the serotonin paradox where individuals with greater (genetically determined) extracellular 5-HT levels also experience depressive symptomology. The neurogenic hypothesis on the other hand, proposes that it is a reduction in neurogenesis and neuroplasticity that underlies depression. However, given that 5-HT and neurogenesis are tightly related, a genetic increase in 5-HT would likely lead to increased neurogenesis (at least early in life) rather than the decreased neurogenesis typically associated with depression. See figure 6.

Therefore, in this study, we will first examine whether rats with a genetically compromised SERT system have altered neurogenesis early in life (PND7). Moreover, as highlighted above, since neuronal development critically depends on maternal 5-HT levels, we will investigate whether the maternal SERT genotype affects neurogenesis. As BrdU immunohistochemistry has been instrumental in confirming that neurogenesis occurs in adult mammalian brains and in examining the effects of antidepressant treatments on neurogenesis (Eriksson et al., 1998; Kuhn et al., 1996; Malberg et al., 2000; Radley \& Jacobs, 2002; Taupin, 2007), this technique will be used to stain new cells in different hippocampal sections (CA1, CA2+CA3 and the DG) to determine the average levels of neurogenesis across genotypes.

Firstly, we hypothesise that the dentate gyrus (DG) will have larger amount of neurogenesis than the $\mathrm{CA} 1$, and $\mathrm{CA} 2+3$ regions as it contains the neural progenitor cells within the neurogenic niche of the SGZ (Altman \& Das, 1966; Zhao et al., 2008).

Secondly, we hypothesis that there will be a gene-dosage dependent increase in neurogenesis, with wildtype (WT, SERT+/+) have the lowest and homozygous (HOM, SERT-/-) rats having the highest levels of newly formed cells. 
Lastly, we hypothesis that the maternal genotype also affects neurogenesis, with HOM mother leading to higher levels of neurogenesis than HET (SERT+/-) or WT mothers.

Figure 6

Factors effecting neurogenesis

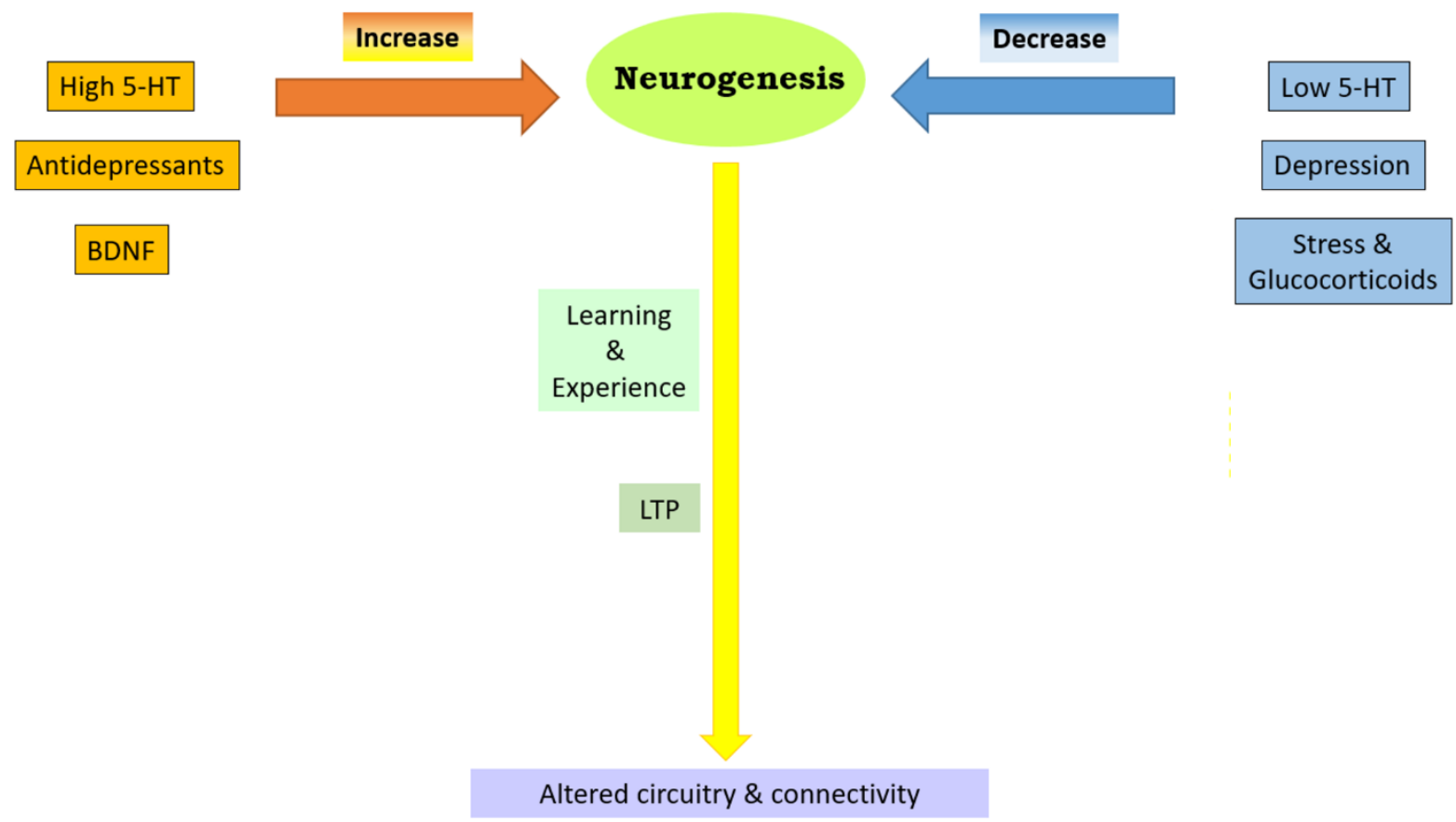

Note. Yellow arrow indicates the hypothesised mechanism underlying the neurogenic and monoamine hypotheses of depression, which is affected by the level of extracellular 5-HT.

In the second part of this study, we planned to investigate more global changes in brain neurochemistry in rats with a genetically compromised SERT system, given the fact that serotonin (and the SERT) has such wide-spread effects on neuronal development early in life. For that, we aim to develop the MALDI protocol which would allow us to visualize and quantify the neuronal distribution of multiple neurochemical compounds simultaneously. This optimization largely focuses on the derivatization of neurotransmitters. Unfortunately, while we made significant progress, we were only able to partially develop the technology as we will discuss in the next chapter. 


\section{Method}

\section{Animals}

This study used Wistar rats which were bred and housed in the Victoria University of Wellington vivarium in accordance with the Animal Welfare Act 1999, with all procedures being pre-approved by the VUW Animal Ethics Committee (AEC number 22375). All animals were housed in polycarbonate cages with one dam and her offspring per cage in a temperature and humidity-controlled environment at $21^{\circ} \mathrm{C} \pm 2{ }^{\circ} \mathrm{C}$ and $55-60 \%$ respectively. A reverse 12-hour light cycle was used with lights on at 1900 - 0700 hours. Food and water were available to the animals ad libitum. To obtain all possible maternal and offspring genotypes, a separate cohort of animals, which consisted of 13 litters of pups bred from seven different genotype pairing combinations, was used. These seven pairings were accomplished by breeding heterozygous (HET; SERT+/-) males with wild type (WT, SERT+/+) homozygous (HOM; SERT-/-) and HET females (genotypes shown in figure 9). Due to time constraints, only male pups were examined (litters: 6, pups: 46).

Figure 9

Offspring genotype combinations

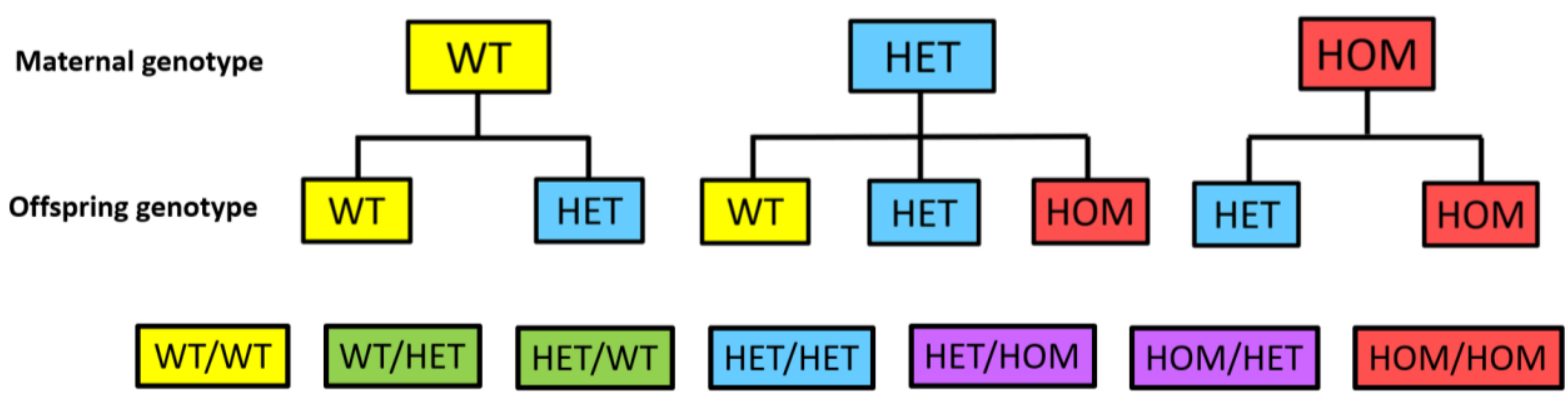

Note. Last row lists 7 possible SERT genotype combinations examined.

\section{Tissue Preparation}

On postnatal day 7 (PND7), pups were removed from cages in pairs and taken into a different room to avoid stressing the remaining animals. Pups were weighed then injected with $25 \mathrm{mg} / \mathrm{kg}$ of BrdU (based on previous pilot work in the VUW Behavioural Neurogenetics lab) intraperitoneally, marked for identification and returned to dams within five minutes. Following a two-hour integration phase, the animals were taken into another room and were deeply anaesthetized with $50 \mathrm{mg} / \mathrm{ml}$ pentobarbital. Once they demonstrated no physical response to a foot pinch, they were rapidly decapitated, and their brains were 
submerged in a $4 \%$ paraformaldehyde solution for 24 hours at $+4^{\circ} \mathrm{C}$ for protein fixing. Tail clippings were taken from each animal to establish their genotypes (Transnetyx, Inc.). Following this, brains were immersed in a $30 \%$ sucrose solution at $+4^{\circ} \mathrm{C}$ for cryoprotection. Once the brains showed no buoyancy (indicating the complete transfer of water for sucrose solution had occurred), the brains were snap frozen at $-40^{\circ} \mathrm{C}$ in isopentane with dry ice. The brains were subsequently stored at $-80^{\circ} \mathrm{C}$ until they were sectioned. All brains were coded to keep the experimenter blind for the remainder of the experiment. $30 \mu \mathrm{m}$ thick coronal sections were sliced using a cryostat with every sixth section used in the analysis to ensure a $180 \mu \mathrm{m}$ distance between sections to avoid counting the same cell multiple times. Slices used in this study were between $-1.20 \mathrm{~mm}$ and $-2.00 \mathrm{~mm}$ from Bregma (Khazipov et al., 2015). Slices were stored in phosphate buffered saline (PBS, $10 \mathrm{mM}, \mathrm{pH} 7.4$ ) with $0.1 \%$ azide at $+4^{\circ} \mathrm{C}$ until processed.

\section{BrdU immunohistochemistry technique principles for new cell labelling}

Bromodeoxyuridine (aka 5-bromo-2'-deoxyuridine, BrdU) is a synthetic thymidine analogue that incorporates into the DNA of dividing cells during the S-phase of the cell cycle (Eriksson et al., 1998; Taupin, 2007). BrdU can be immunohistochemically detected (Gratzner, 1982; Nagashima, DeArmond, Murovic \& Hoshino, 1985) in vitro and in vivo, and as BrdU is incorporated into newly synthesised DNA, an antibody can then be used to visualise newborn cells that were dividing during this BrdU exposure (Duman, 2004; Magavi \& Macklis, 2008). BrdU is therefore utilized to monitor birth dating and cell proliferation (Taupin, 2007). BrdU immunohistochemistry is advantageous as it is highly reproducible and quickly detected while the previously used tritiated thymidine autoradiography method is more variable and typically requires at least weeks of exposure (Magavi \& Macklis, 2008). BrdU exposure as short as 6 minutes is detectable (Graztner, 1982). BrdU labelling can also be used for thicker slices $40-50 \mu \mathrm{m}$ thick while tritiated thymidine can only detect labelled cells in the topmost 3-5 $\mu \mathrm{m}$ of sections (Magavi \& Macklis, 2008). BrdU provides very low background intranuclear labelling and immunohistochemical detection up in sections up to $35 \mu \mathrm{m}$ thick (Markakis \& Gage, 1999). After a short period (approximately two hours after BrdU administration) BrdU-immunopositive cells are found in clusters in the SGZ between the granule cell layer and the hilus (Duman, 2004). Typically, two hours is sufficient for BrdU uptake but not mitosis or migration (Gould et al., 1998) and therefore labels cells in the proliferation stage of neurogenesis, however depending on when $\mathrm{BrdU}$ is administered it can 
also illustrate cell differentiation, migration and survival (Abrous et al., 2005). Thus, BrdU immunohistochemistry is the method of choice for labelling newly generated neurons (Ngwenya, Peters \& Rosene. 2005).

BrdU immunohistochemistry is performed with the Avidine-Biotine Complex (ABC) method (shown in figures 7 and 8). The ABC method utilizes the high affinity of Avidin, a glycoprotein, for Biotin, a low molecular weight vitamin (Bratthauer, 2010). Large amounts of biotin can be easily conjugated to antibodies and enzymes via covalent attachment (Bratthauer, 2010). While the common ABC method uses a primary and secondary antibody (the three step ABC method), some primary antibodies such as the one used in this study is biotinylated itself and reacts with an avidin-biotin-peroxidase complex of avidin bound to biotinylated peroxidase to amplify this signal, which eliminates the need for a secondary biotinylated antibody (the two step ABC method; Bratthauer, 2010; Hsu, 1990). Peroxidase has an oxidative function which allows for the use of chromogens that change colour when oxidized (Bratthauer, 2010). Thus, when hydrogen peroxide is added, it is degraded by the peroxidase and releases oxygen that goes on to react with (oxidise) 3,3'-diaminobenzidine (DAB) (Bratthauer, 2010) and nickel chloride which changes its colour from a light pink in solution to a purple-black that allows the visualisation of the antibody-antigen reaction. However, as commercial antibodies only recognise BrdU in single-stranded DNA, the DNA need to be unwound into single strands with hydrochloric acid ( $\mathrm{HCl})(\mathrm{Ngwenya}$ et al., 2005). The tissue is therefore preserved in paraformaldehyde, which provides enough fixation of brain tissue and proteins that the $\mathrm{HCl}$ treatment does not compromise tissue integrity (Ngwenya et al., 2005). This method is one of the most widely used immunocytochemistry methods used today (Bratthauer, 2010). 
Figure 7

BrdU and two-step ABC immunohistochemistry

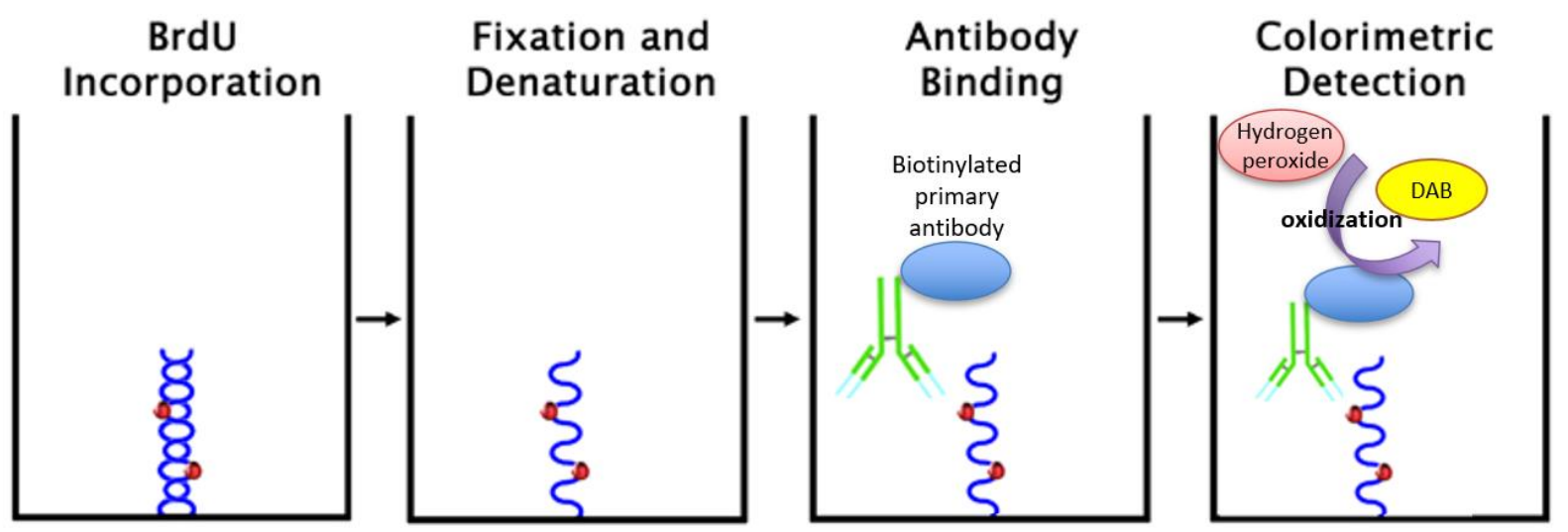

Note. Modified from Creative Bioarray. (n.d.).

Figure 8

The two-step Avidin-Biotin labelling technique

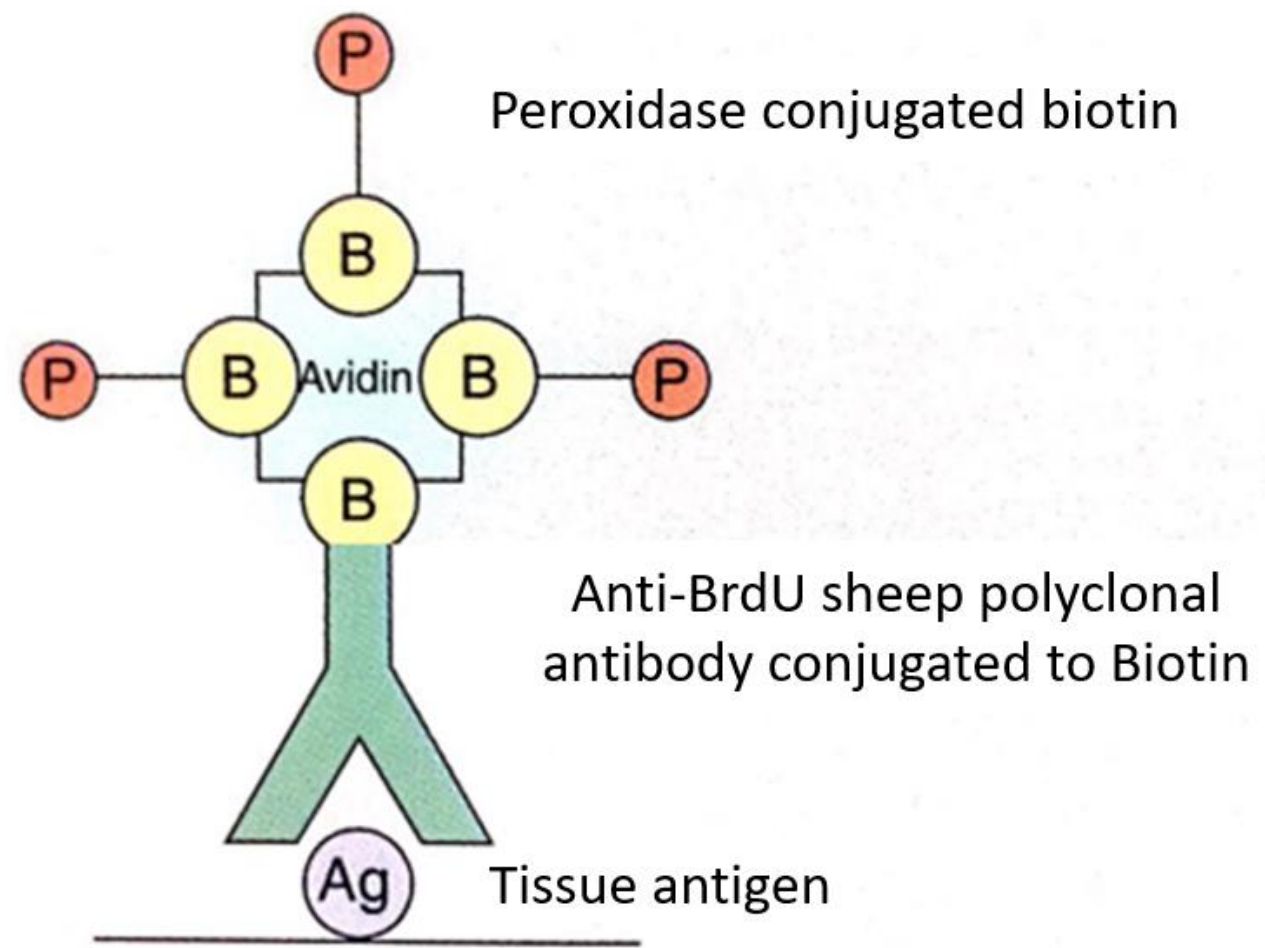

Note. Modified from Slap (2002). 


\section{BrdU Labelling Procedure}

The ABC peroxidase method for labelling BrdU-positive cells in free floating sections, a procedure previously established within the VUW behavioural neurogenetics lab, was used in this experiment. All steps were carried out under mild agitation and at room temperature. Sections were removed from storage and washed for five minutes, three times in PBS which was replaced between each wash. An antigen retrieval process was used to denature the DNA strands to allow access for BrdU. This was achieved by incubating the sections for 90 minutes in $5 \mathrm{~mL}$ of $2 \mathrm{~N} \mathrm{HCl}$. Following this incubation, sections were washed three times for five minutes as stated above. Sections were then incubated in $1 \%$ Bovine Serum Albumin (BSA; Sigma-Aldrich) in a PBST solution (created by adding Triton X-100 [ThermoFisher] to PBS) for 60 minutes to increase membrane permeability. Sections were then incubated in 3\% hydrogen peroxide $(\mathrm{H} 2 \mathrm{O} 2)$ in a PBS solution for 30 minutes (to quench endogenous peroxidase and prevent non-specific peroxidase-based background staining) then washed in PBS three times for five minutes as above. Sections were then incubated overnight in the primary anti-BrdU sheep polyclonal antibody, conjugated to Biotin, ab2284 (Abcam) diluted in PBST and 1\% BSA. Following this, sections were washed once again in PBST as above then incubated in Avidin-biotin peroxidase complex (ABC; Vectastain) diluted 1:1000 in PBST (prepared 30 minutes beforehand) for 60 minutes. Following another washing in PBST as above, sections were incubated in a DAB $(0.2 \mathrm{mg} / \mathrm{mL})$ and Nickel chloride $(\mathrm{NiCl} 2$, $0.4 \mathrm{mg} / \mathrm{mL})$ solution prepared in Tris $\mathrm{HCl}$ buffer $(50 \mathrm{mM}, \mathrm{pH} 7.4)$ with $0.3 \% \mathrm{H} 2 \mathrm{O} 2$, to induce oxidization of the DAB. Thus, peroxidase breaks down $\mathrm{H} 2 \mathrm{O} 2$, which releases oxygen, which then oxidises with $\mathrm{DAB}$ to give a brown (DAB alone) or blackish $(\mathrm{DAB}+\mathrm{NiCl} 2)$ precipitate, producing coloured staining of the BrdU sites. Sections were washed once more in PBST as above.

Following staining, sections were stored at $4^{\circ} \mathrm{C}$ overnight until mounting within 48 hours. Stained sections were extracted from their baskets and immersed in PBST and gently mounted onto gelatinised slides and allowed to dry for at least 30 minutes. After mounting, counterstaining was undertaken to provide a stronger contrast between BrdU positive cells and the surrounding tissue. This involved immersion of the mounted sections in $1 \%$ neutral red ( $\mathrm{pH} 4.8$ ) solution (Biovision) for two minutes, followed by a brief immersion in a progressively increasing strength of ethanol: 70\%, 90\% twice, then 100\% twice. Following this, sections were immersed in two baths of Histo-Clear (National Diagnostics). Lastly, a coverslip was applied with DPX (Sigma-Aldrich) and allowed to dry for at least 48 hours. 
Manual counts of BrdU-labelled cells were performed blind using the software Neurolucida (MBF Bioscience; Williston, VT, USA) and Olympus BX51 microscope (Olympus; Wellington, New Zealand) with a motorized x-y stage and a Lumenera_camera (Teledyne Lumenera, Ottawa, Canada) at a 20x magnification. The DG, CA1 and CA2+CA3 regions were manually traced first at 10x magnification using a mouse-controlled cursor on a digital image of the slice to obtain the areas for each region. Cell densities were calculated by dividing the total number of cells counted in each of the three hippocampal regions by the total area of that region for each slice (bilaterally). 


\section{Results}

The data were analysed with IBM SPSS Statistics (version 25). Data was approximately normally distributed, assessed visually using a Q-Q plot. Levene's $F$ tests were significant therefore we could not assume equal variances, so estimated marginal means and robust standard errors bars are used when presenting the results. Outliers from three brains (2 offspring HET/maternal WT, 1 offspring WT/maternal HET) were removed where little to no cells were detected.

As the homogeneity of variances was not met and as not every offspring genotype and maternal genotype combination was observed, data was initially analysed using three twoway factorial ANOVAs with Type IV sums of squares, after discussion with a statistician. These ANOVAs were conducted to assess the influence of the independent variables, offspring SERT genotype and maternal SERT genotype, on cell density for each of the three hippocampal regions to assess whether there were any mean cell density differences between offspring and maternal genotypes. The offspring genotypes had three levels (WT, HET, HOM) as did maternal genotype (WT, HET, HOM) for each region.

If the differences between offspring genotypes were found to differ between maternal genotypes (i.e., a significant interaction term), then this analysis was followed up with planned comparisons to compare maternal genotype differences within each offspring genotype. This was done using either an independent-samples t-test or one-way ANOVA as appropriate, and the Bonferroni correction was applied to correct for multiple comparisons such that $p$-adj denotes Bonferroni-adjusted $p$-values. If the differences between offspring genotypes were not found to differ between maternal genotypes, Bonferroni-adjusted pairwise comparisons of the estimated marginal means $\left(M^{*}\right)$ were used to determine differences between genotypes and maternal genotypes. 
Figure 10

Coronal section from a BrdU-stained HET x HOM brain at PND7
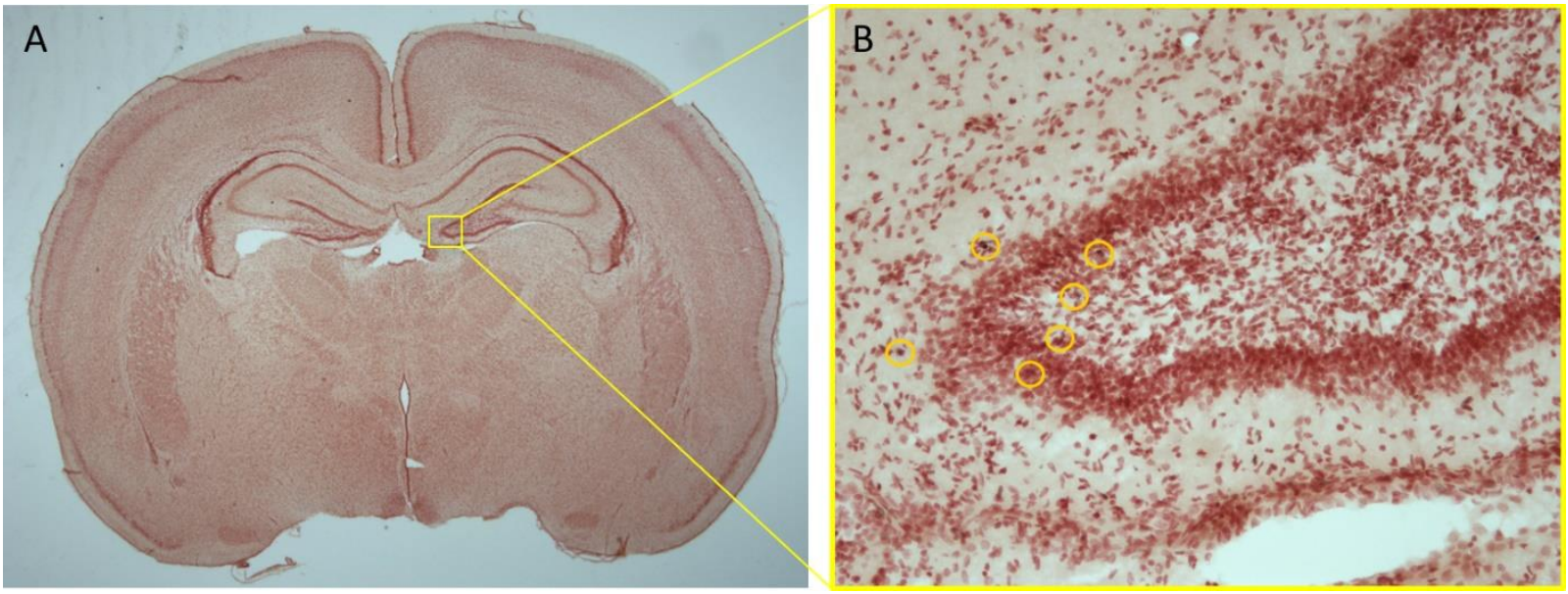

Note. Coronal section in at Bregma $-1.60 \mathrm{~mm}$ to $-1.80 \mathrm{~mm}$ in A (Khazipov et al., 2015). BrdUlabelled cells in the DG in B.

Means and standard deviations of the total area $\left(\mathrm{mm}^{2}\right)$ of all three hippocampal sections were computed for DG, CA1 and CA2 + CA3 to determine if there was equivalence of the hippocampal sizes between offspring and maternal genotype groups (see Appendix A).

Equivalence of the size of the mean area for each hippocampal region across offspring and maternal genotypes was determined using two-way analyses of variance (ANOVAs) which revealed no offspring or maternal genotype differences for DG, CA1 or CA2+CA3 mean area (See appendix).

After confirmation of size equivalence, means and SDs for BrdU-positive cell density were calculated (table 1). 
Table 1

Density of BrdU-positive cells (number of per $\mathrm{mm}^{2}$ ) for the three hippocampal

\begin{tabular}{|c|c|c|c|c|c|c|c|c|}
\hline \multirow{2}{*}{$\begin{array}{l}\text { Offspring } \\
\text { genotype }\end{array}$} & \multirow{2}{*}{$\begin{array}{l}\text { Maternal } \\
\text { genotype }\end{array}$} & \multicolumn{2}{|c|}{ DG } & \multicolumn{2}{|c|}{ CA1 } & \multicolumn{2}{|c|}{$\mathrm{CA} 2+\mathrm{CA} 3$} & \multirow[b]{2}{*}{$n$} \\
\hline & & $M$ & $S D$ & $M$ & $S D$ & $M$ & $S D$ & \\
\hline \multirow[t]{2}{*}{ WT } & WT & .55 & .08 & .21 & .07 & .26 & .06 & 4 \\
\hline & HET & .32 & .21 & .11 & .08 & .16 & .14 & 8 \\
\hline \multirow[t]{3}{*}{ HET } & WT & .43 & .21 & .15 & .09 & .19 & .12 & 9 \\
\hline & HET & .44 & .17 & .16 & .08 & .18 & .08 & 9 \\
\hline & HOM & .43 & .21 & .16 & .07 & .20 & .06 & 4 \\
\hline \multirow[t]{2}{*}{ HOM } & HET & .53 & .14 & .21 & .09 & .25 & .08 & 3 \\
\hline & HOM & .59 & .11 & .24 & .05 & .28 & .07 & 9 \\
\hline
\end{tabular}

Next a one-way Welch's ANOVA was conducted to assess whether BrdU-positive cell density differed by hippocampal region (Delacre, Leys, Mora \& Lakens, 2018) and showed a significant main effect $[F(2,213.08)=104.93, p<.001]$ indicating that cell density did differ across hippocampal regions. Post hoc comparisons using the GamesHowell test were conducted to identify the origin of the differences. All three areas differed significantly to each other with the DG having the highest cell density followed by the CA1 and lastly the CA2+CA3 (See table 1 for means and SDs).

\section{Neurogenesis in the $D G$}

A two-way ANOVA was conducted to assess the influence of maternal SERT genotype and offspring SERT genotype on cell density for the DG. We found a significant interaction between maternal and offspring genotype for mean cell density in the DG $[F(2$, $110)=4.01, p=.021]$. This indicates that the genotype difference in cell density depends on the maternal genotype. There was a significant genotype effect $[F(2,110)=4.027, p=.021]$, 
with a significant difference between HET $\left(M^{*}=.429, S E=.024\right)$ and $\mathrm{HOM}\left(M^{*}=.558, S E\right.$ $=.040, p=.021)$, indicating HOMs had higher mean cell density within the DG. The difference between HOM and WT $\left(M^{*}=.432, S E=.033\right)$ was approaching significance $(p=$ $.052)$ while there was no significant difference between WT and HET ( $p=1.00)$. The effect of maternal genotype was not significant $[F(2,110)=.115, p=.891]$. See figure 11A.

Planned comparisons revealed that the mean difference in cell density between maternal WT and HET genotypes within WT offspring was significant in the DG $[t(26.152)=$ 4.248, $p$-adj <.001], but was not significant between HET and HOM genotypes within HOM offspring $[t(6.710)=-.904, p-a d j=1.191]$, or between WT, HET and HOM genotypes within HET offspring $[F(2,55)=.019, p-a d j=.2 .943]$.

Figure 11

Bar charts of mean new cell density for hippocampal DG, CA1 and CA2+CA3 regions.

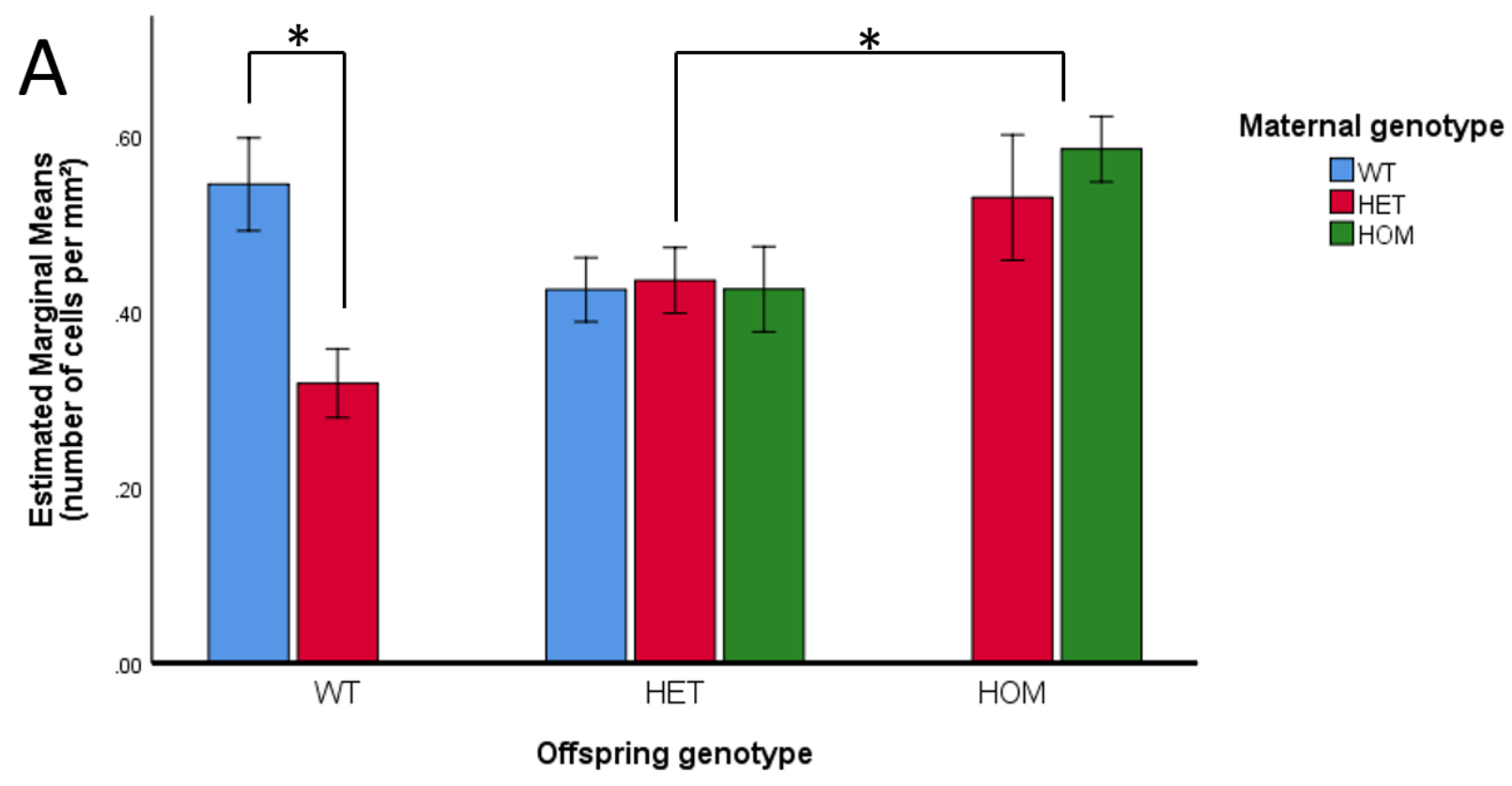



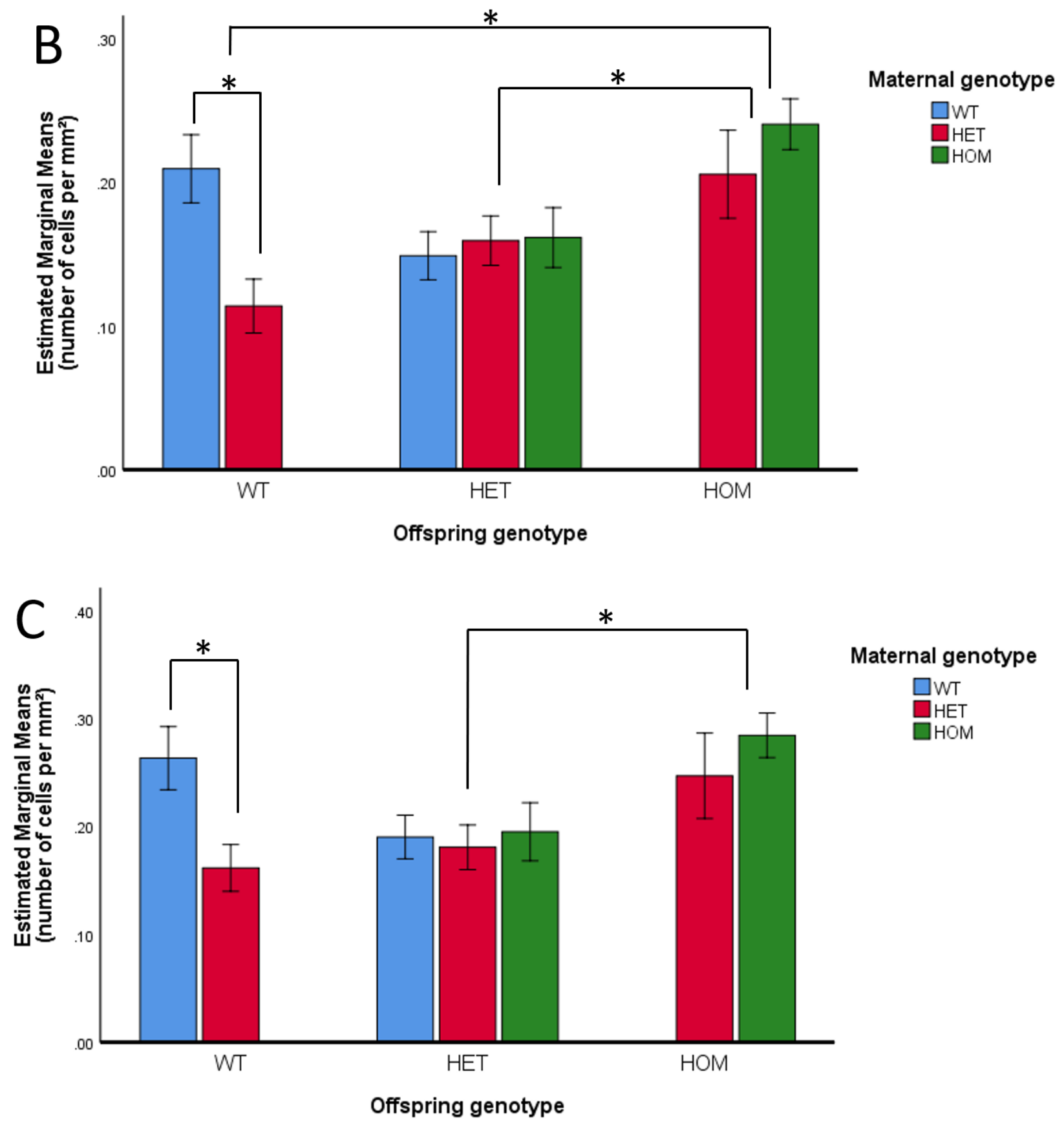

Note. A. B and C correspond to DG, CA1 and CA2+CA3 respectively. Error bars represent \pm 1 standard error of the means. * Denotes significant differences.

\section{Neurogenesis in the CA1}

Next, a two-way ANOVA was conducted to assess the influence of maternal SERT genotype and offspring SERT genotype, on cell density for the CA1. There was also a significant interaction between maternal and offspring genotype in CA1 mean cell density $[F$ $(2,95)=3.808, p=.026]$, indicating that in the CA1, maternal genotype affects the 
difference in mean cell density in offspring genotype (figure 11B). There was a significant genotype effect $[F(2,95)=4.579, p=.013]$, with a significant difference between WT $\left(M^{*}\right.$ $=.162, S E=0.015)$ and $\operatorname{HOM}\left(M^{*}=0.223, S E=.018, p=.029\right)$ with HOMs having a higher mean cell density than WT in the CA1. There was also a significant difference between HET $\left(M^{*}=.157, S E=.011\right)$ and HOM $(p=.005)$ with HOMs having higher cell density again, but no significant difference between WT and HET $(p=1.00)$. The effect of maternal genotype was not significant $[F(2,95)=.362, p=.697]$.

The mean difference in cell density between maternal WT and HET genotypes within WT offspring was significant in the CA1 $[t(20.663)=3.199, p-a d j=.012]$ but not significant between maternal HET and HOM genotypes within HOM offspring $[t(6.069)=-.922, p$-adj $=$ 1.176], or between maternal WT, HET and HOM genotypes within HET offspring CA1 $F(2$, 49) $=.126, p-a d j=2.646]$.

\section{Neurogenesis in the $C A 2+C A 3$}

Lastly, a two-way ANOVA was conducted to assess the influence of maternal SERT genotype and offspring SERT genotype, on cell density for the CA2+CA3 region. For the CA2+CA3 region the interaction was not significant $[F(2,95)=1.973, p=.144]$. There was a significant genotype effect within the $\mathrm{CA} 2+\mathrm{CA} 3$ region $[F(2,95)=3.868, p=.024]$, with a significant difference between HET $\left(M^{*}=.189, S E=.013\right)$ and $\operatorname{HOM}\left(M^{*}=.266, S E=.022\right.$, $p=.011)$ where HOMs had a higher mean cell density, but no significant difference between WT $\left(M^{*}=.212, S E=.018\right)$ and HET $(p=.881)$ or WT and HOM $(p=.201)$. The effect of maternal genotype was not significant $[F(2,95)=.454, p=.636]$. See figure $11 \mathrm{C}$.

The mean difference in cell density between maternal WT and HET genotypes within WT offspring was significant in CA2+CA3 $[t(28.269)=2.854, p$-adj $=.024]$ but not significant between maternal HET and HOM genotypes $[t(7.293)=-1.064, p$-adj $=.963]$ or between maternal WT, HET CA2+CA3 $[F(2,35.792)=.185, p-a d j=2.496]$. 


\section{Discussion}

This experiment aimed to examine the effects of both offspring and maternal SERT genotype on early postnatal neurogenesis in the hippocampus. While most studies have focused on the genotype of the individual, the maternal SERT genotype's effect on early neurogenesis has been less studied. It was hypothesised that the DG would have the highest cell density of the hippocampal regions. Second, it was hypothesised that the HOM offspring group would have the greatest density of newly formed cells, followed by the HET then the WT groups. Third, it was hypothesised that the maternal HOM would have higher cell density than the maternal HET and WT groups across the offspring genotypes.

Firstly, we found that the DG had the highest newborn cell density of the three hippocampal subfields which supported the first hypothesis. This is consistent with studies showing that the DG contains the neurogenic SGZ (Altman \& Das, 1966; Alvarez-Buylla \& García-Verdugo, 2002; Duan et al., 2008; Duman, 2004; Ehninger \& Kempermann, 2008; Eriksson et al., 1998; Gage, 2002; Kempermann et al., 2004a, 2004b; Kuhn et al., 1996; Vadodaria \& Jessberger, 2014; van Praag et al., 2002; Zaverucha-do-Valle et al., 2013; Zhao et al., 2008).

Secondly, our findings indicate that offspring SERT genotype influences neurogenesis in early life, with HOMs having higher newborn cell densities than HETs and WT. This partly supports our second hypothesis as HOMs consistently had the highest newborn cell density in all three regions. However, the differences were only between HOM and HETs in all regions and between HOM and WTs in the DG and CA1. WTs were not significantly different than HETs in the CA1 and CA2+CA3 regions or from HOMs in the CA2+CA3. Nonetheless, WTs had lower levels of newborn cell density than HOMs in all regions.

This is consistent with previous findings that altered amounts of 5-HT during development affects neurodevelopment (e.g., Faber \& Haring, 1999; Matsukawa et al., 2003; Massart et al., 2012; Santarelli et al., 2003; Vitalis et al., 2007). As HOM offspring showed higher cell density than HETs and WTs the findings are partially consistent with previous research illustrating that a genetic inactivation of SERT and its resulting increased 5-HT levels, leads to increased neurogenesis (Karabeg et al., 2013). 
While our hypothesis was not fully supported, it should be noted that virtually all research (behavioural and otherwise) are typically performed in offspring of HET/HET breeding. Inspection of the results show that in these animals we do find the gene-dosage dependent increase in neurogenesis in all three hippocampal subregions. The non-significant result may be a result of the unequal variances and a lack of statistical power as some genotype groups had very small numbers.

Lastly, we found significant interactions between the maternal and offspring genotype in the DG and CA1 regions, indicating that in these regions the maternal genotype influences newborn cell density. Further analysis revealed maternal genotypes only differed in WT, where WT offspring from maternal WTs had greater newborn cells than WT offspring of maternal HET. On the other hand, there were no maternal genotype effects for HET or HOM offspring. Thus, these interactions were driven by maternal genotype differences within WT offspring, leading to WT animals with HET mothers have lower neurogenesis than WT animals with WT mothers.

To the best of our knowledge, this is the first time a maternal genotype effect on neurogenesis has been described and, as such, it is very difficult to interpret, particularly as this effect was only seen in WT offspring rats. It can be speculated, however, that the maternal HETs have (albeit slightly) higher extracellular serotonin levels which may have affected foetal development. As discussed in the introduction, early in development, 5-HT levels in the foetus are mainly driven by maternal supply. This excess maternal 5-HT during development may 'program' WT offspring neurodevelopment to prepare them for their environment, as seen in Heiming et al. (2009). Excess maternally supplied 5-HT may, for example, activate or even upregulate the offspring's 5-HT1A autoreceptors to decrease serotonergic cell firing and decrease extracellular 5-HT. This effect is certainly seen in SERT knockout mice and after SSRI treatment (Fabre et al., 2000; Le Poul et al., 2000). Given that HET and HOM offspring are likely to have downregulated 5-HT1A receptors, the influence of the maternal genotype (and the resulting changes in 5-HT levels) may have been blunted in these animals.

In addition to the maternal genotype dependent differences in perinatal 5-HT levels, it is important to realize that all brains were taken one week after birth, suggesting that 
differences in maternal care may also have played a role in the maternal genotype dependent changes in neurogenesis.

Poor maternal care is a stressful early life experience which influences the emotional, behavioural and cognitive outcomes of the offspring. Overall, insufficient maternal care exacerbates stress responses, leads to more depressive and anxious behaviours and alters serotonergic signalling (Benekareddy, Goodfellow, Lambe \& Vaidya, 2010, Benekareddy, Vadodaria, Nair \& Vaidya, 2011; Bravo, Dinan \& Cryan, 2014; Houwing, Ramsteijn, Riemersma \& Olivier, 2019). For example, maternal separation in rats reduced raphe 5-HT concentration and SERT expression and alters 5-HT1A and 5-HT2 receptor expression (Benekareddy et al., 2010, 2011; Bravo et al., 2014; Lee et al., 2007; Vázquez, Eskandari, Zimmer, Levine \& López, 2002; Vicentic et al., 2006).

Studies have also shown that there is a link between maternal SERT genotype and maternal care behaviour as human $s$ allele carriers and SERT knockout rodents demonstrate altered maternal caregiving (Bakermans-Kranenburg \& van Ijzendoorn, 2008; Heiming et al., 2013; Johns et al., 2005; Mileva-Seitz et al., 2011; Winokur, Lopes, Moparthi \& Pereira, 2019). Altered maternal caregiving might arise in genetically deficient SERT knockout models of depression through altered hippocampal function. For example, in the hippocampus, 5-HT affects the regulation of oxytocin which plays a crucial role in parenting and maternal care (Bakermans-Kranenburg \& van Ijzendoorn, 2008; Feldman \& BakermansKranenbur, 2017; Jørgensen, Riis, Knigge, Kjær \& Warberg, 2003; Johns et al., 2005). In line with this, Kikusui, Ichikawa and Mori (2009) found that a lack in mother-pup interaction during late lactation leads to increases corticosterone synthesis, reduced BDNF and reduced neurogenesis while Liu, Diorio, Day, Francis and Meaney (2000) find greater maternal care to be associated with increases synaptogenesis or synaptic survival. Maternal care-induced offspring outcomes are also affected by offspring's genotype which may confer a sensitivity to (early) environmental stress for a genetically vulnerable group (Drury et al., 2012; Madrid et al., 2018).

We can therefore speculate that maternal care may be a factor that affected neurogenesis in our study. Based on our findings that WT/WT offspring had neurogenesis levels higher than WT/HETs while no other maternal genotype influences were detected, we would hypothesise that a WT mother may provide better maternal care than HET or HOM 
mothers. It could be that the lower neurogenesis observed in WT offspring from HET mothers compared to WT/WTs is due to poorer maternal care by HET mothers. If so, this may mean the WT animals may have a neurodevelopmental sensitivity for early life experiences as seen in previous research (Drury et al., 2012; Madrid et al., 2018). The lack of maternal differences in HET and HOM offspring would, conversely, suggest a blunted response to early life experiences in these animals.

Research also needs to be done examining interactions between maternal care behaviour and offspring genotypes, to determine whether a WT mother displays differences in care behaviour for a WT versus a HET offspring. If so, further studies need to examine the effects of cross-fostering HET and HOM offspring with WT mothers to determine how this effects offspring neurogenesis.

\section{Theoretical implications}

Although the maternal genotype/offspring genotype interaction are difficult to explain at this point in time, it is important to note that virtually all previous research on SERT knock-out rats and mice have been done using HET/HET breeding pairings. This includes the research on the biochemical changes observed, as well as the increase in depressive and anxiety-like behaviours in these offspring (Homberg et al., 2007; Olivier et al., 2008). In the current study we found that, for this pairing, there was a gene-dosage dependent increase in offspring neurogenesis and therefore, the following implications are discussed for the main effect of an offspring genotype-dependant increase in neurogenesis.

These results demonstrate that through genetically increasing extracellular 5-HT in development, neurogenesis is increased. The monoamine hypothesis postulates that lower adult levels of monoamines, particularly serotonin, underlie the neurobiology of depression and anxiety, and this is associated with lower levels of adult neurogenesis (Boldrini et al, 2013; Bremner et al., 2000; Brezun \& Daszuta, 1999, 2000a; Campbell et al., 2004; Campbell \& MacQueen, 2004; Kronmüller et al., 2009; Parihar, Hattiangady, Shui \& Shetty, 2013; Sheline et al., 1996; Stockmeier et al., 2004; Videbech \& Ravnkilde, 2004). In line with this hypothesis, conditions associated with increased neurogenesis are associated with increases in 5-HT signalling (Dekeyne et al., 2008; Kim et al., 2012; Klempin et al., 2013), and 
conversely, conditions associated with decreased neurogenesis such as aging, high corticosterone levels, stress and malnutrition also alter the serotonergic system (Blatt et al., 1994; Chalmers et al., 1993; Gould, 1999; McKittrick et al., 1995; Míguez et al., 1999; Neumaier et al., 1997; Watanabe et al., 1993). Thus, serotonergic signalling appears to be critically involved in neurogenesis, and our data are consistent with the stimulatory role of 5HT on neurogenesis. This effect is likely mediated (at least in part) by BDNF as this protein regulates neurodevelopment and plasticity in neural circuits (Mattson, Maudsley \& Martin, 2004; Pascual-Brazo et al., 2012; Scharfman et al., 2005) and is also enhanced by 5-HT (Kronenberg et al., 2016). Studies showing that SSRIs increase BDNF expression also suggest a role for BDNF in antidepressant action (Brunoni, Lopes \& Fregni, 2008; Duman \& Monteggia, 2006; Martinowich \& Lu, 2008).

Our findings are also consistent with the paradoxical effects of serotonin. While in adulthood depression seems to be associated with decreased 5-HT and decreased neurogenesis, our data show that early life increases in 5-HT are associated with increased neurogenesis in rats that in adulthood develop symptoms of depression and anxiety. These data are in line with other animal research emphasizing the 5-HT paradox. Overall, stress models of depression find reductions in adult hippocampal neurogenesis and neuroplasticity (Chen et al., 2010; Djordjevic et al., 2012; Frodl et al., 2010; Jacobs et al., 2000; Jayatissa, Bisgaard, Tingström , Papp \& Wiborg, 2006; Lee et al., 2006; Luo et al., 2005; Perlman et al., 2012; Vollmayr et al., 2003; Zeng et al., 2012), while SERT knock out models exhibit an increase in neurogenesis (Ferrés-Coy et al., 2013; Karabeg et al., 2013; Schipper, Kiliaan \& Homber, 2011).

The difference between the two types of depression models studied, however, is the fact that SERT knock out affects hippocampal connectivity starting at development while stress models of depression induce depression without this early alteration of neural connectivity taking place. We propose that this difference is the basis for the 5-HT paradox, where depression in genetically SERT compromised individuals versus non-compromised individuals arises via different underlying mechanims, ultimately leading to two (or more) different types of depression. In this respect, it is important to realize that neurogenesis during development and in adulthood are fundamentally different processes, as alluded to in the introduction. It is therefore paramount to investigate whether HOMs and HETs also have 
altered neurogenesis in adulthood, and, if so, whether adult neurogenesis is increased or decreased, as well as the associated depressive outcomes.

Irrespective of this, neurogenesis is only the first step in neuronal plasticity. 5-HT regulates other developmental processes, such as synaptogenesis, spine genesis and axon guidance, where alterations in 5-HT signalling result in aberrant growth, connectivity and circuitry (Chen et al., 2010; Chugani, 2002; Côté et al., 2007; Faber \& Haring, 1999). In addition, we do not know whether these new cells develop into neurons or glial cells and whether or how these are integrated in the hippocampal circuitry remains to be investigated.

Several studies do show that newly generated neurons are functional additions to hippocampal circuitry as they form axonal projections, make synaptic connections and have the same electrophysiological characteristics of neighbouring mature cells in neurotypical animals (Markakis \& Gage, 1999; van Praag et al., 2002). However, at least in cell cultures, HOM rats have reduced spine formation (Chaji et al. 2021). Thus, it is conceivable that even if more newborn cells develop into more neurons in HET and HOM offspring, they may not correctly synapse onto their postsynaptic targets. The neurogenesis seen in HOM animals could thus result in aberrant neural connectivity. We suggest that the connections their new neurons might make are likely to be altered from normal development because they are a result of dysregulated 5-HT levels. Indeed, proper synaptogenesis and the development of neurocircuitry requires a critical concentration of 5-HT while many aspects of neurodevelopment are altered in SERT knockout and SSRI treated rodents (Azmitia, 2001; Brezun \& Daszuta, 1999; 2000a, 2000b; Chen et al., 2010; Chugani, 2002; Vitalis et al., 2007; Vitalis \& Parnavelas, 2003).

As the hippocampus plays an esssential role in emotion regulation (Kirkby et al., 2018), alterations in the connectivity of the hippocampus is likely to alter emotion regulation. Indeed, mood dysregulation, including depressive behaviour, is associated with hippocampal dysregulation including reductions in neurogenesis, neuron loss and hippocampal volume loss (Boldrini et al, 2013; Bremner et al., 2000; Campbell \& MacQueen, 2004; Kronmüller et al., 2009; Parihar et al., 2013; Sheline et al., 1996; Stockmeier et al., 2004; Videbech \& Ravnkilde, 2004). Animal studies also find dramatic alterations in the neurocircuitry of SERT knockout animals, such as that of the limbic cortical-ventral striatopallidal reward pathway 
(Bearer, Zhang, Janvelyan, Boulat \& Jacobs, 2009; Daubert \& Condron, 2010). Depressed individuals clearly have altered/disrupted neural connectivity, especially within the hippocampus (Chase et al., 2014; Cullen et al., 2009) and show impairments in other hippocampal dependent functions (Austin et al., 2001; Becker et al., 2009; Bremner et al., 2004; Burt et al., 1995; Ilsley et al., 1995; MacQueen et al., 2003). Therefore, we suggest that the increased neurogenesis in HOM animals in this study is likely to alter their hippocampal regualtion of emotional behaviours which would align with the previous findings that SERT knockout rats have demonstrated altered emotonality and behaviour previously (Kalueff et al., 2010; Olivier et al., 2008).

We therefore propose that the differential effects of 5-HT in the 5-HT paradox are brought about through early-life increases in 5-HT in both animal models and $s$ allele carriers, altering their hippocampal neurocircuitry during development. We propose that it is this early altered hippocampal connectivity that leads to altered emotion regulation in genetically vulnerable depressed models and individuals. On the other hand, we hypothesise that in individuals without this genetic reduction in SERT, the induction of depression in later life by stressful life experiences alters their neural circuitry, but does so through a stressinduced loss of connectivity which is restored via antidepressant treatment (see figure 12).

Figure 12

The proposed neurobiological etiology of neurogenic vs stress-induced depression

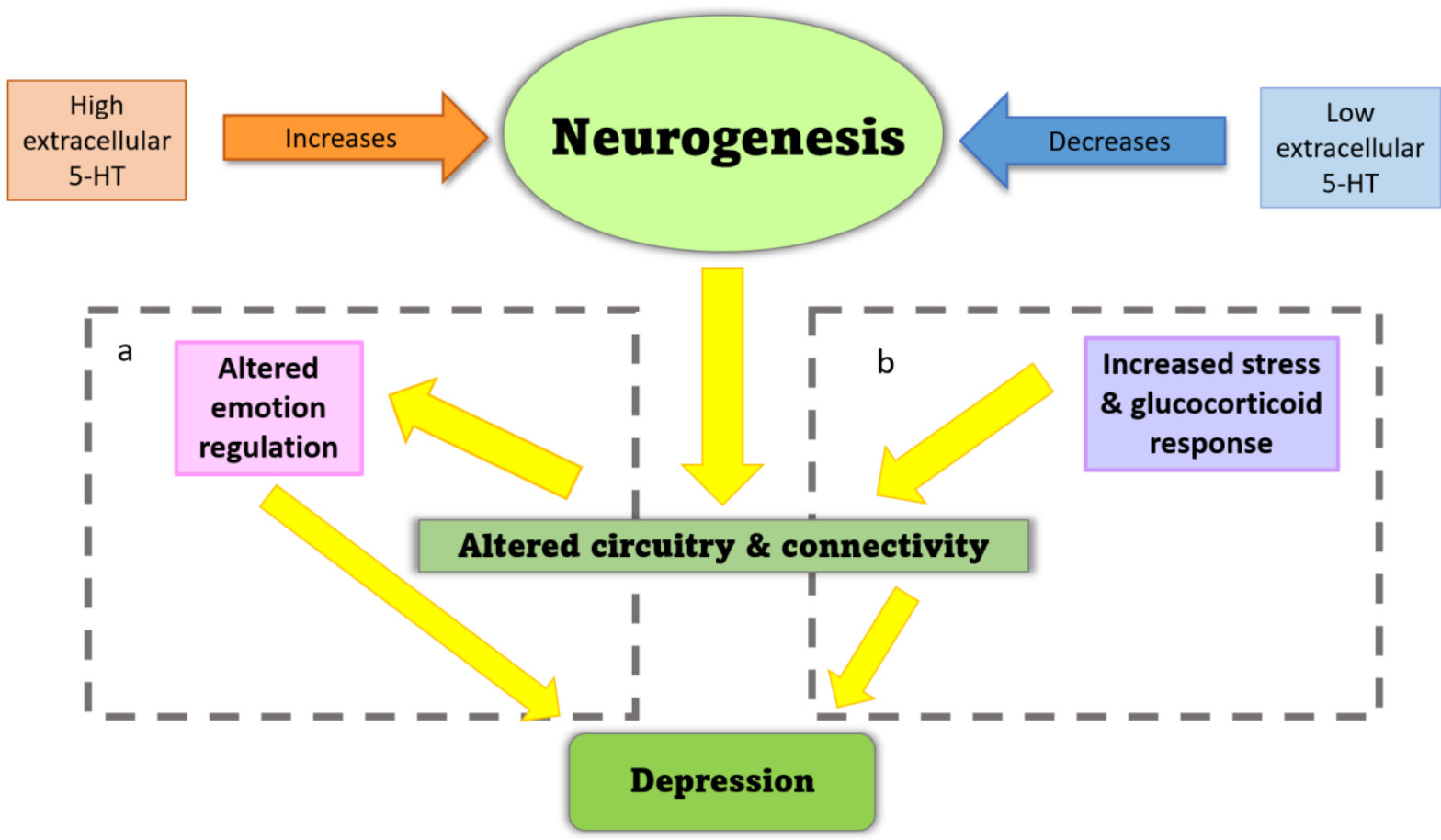


Note. A shows the neurogenic model of depression which occurs due to developmental serotinergic changes and B shows the proposed mechanism of stress induced depression.

Experience and learning throughout the lifespan underlie sensory plasticity and involve the regulation of synaptic formation, removal and morphological remodelling of synapses and dendritic spines (Feldman, 2009; Holtmaat \& Svoboda, 2009; Teissier et al., 2017; Trachtenberg et al., 2002; Wilbrecht et al., 2010). An important aspect of the neurogenic hypothesis that needs further study is the effect increasing neurogenesis has on learning and the response to stressful experiences. Theories regarding the role of adult neurogenesis in hippocampal function suggest a role in learning, or to allow the DG to accommodate continual modulation and information-processing demands (Gould et al., 1999a; Kempermann, 2002). Indeed, increasing neurogenesis benefits learning (Mattson et al., 2004; Sahay et al., 2011) while reducing neurogenesis impairs learning (Hu et al., 2009; Kim et al., 2012; Mohapel et al., 2005; Saxe et al., 2006; Shors et al., 2002; Winocur et al., 2006; Zhang et al., 2008). Supporting this notion, studies have shown that disruption of neurogenesis also leads to significant memory and learning impairments and that altered hippocamapal functioning in mood disorders is also associated with cognitive and learning impairments (Becker et al., 2009; Bremner et al., 2004; Deckersbach et al., 2006; Gourevitch, Rocher, Pen, Krebs \& Jay, 2004; Moser et al., 1995; Moser \& Moser, 1998; Snyder, Hong, McDonald \& Wojtowicz, 2005).

The neurogenic hypothesis postulates that it is the structural plasticity associated with enhanced neurogenesis that is compromised in depression. Indeed, structural plasticity, particularly in synapses, connectivity and receptors, is involved in depression (Albert \& Lemonde, 2004; Cai et al., 2013; Ferrés-Coy et al., 2013; Fuchs et al., 2004; Kaiser et al., 2015; Lesch \& Waider, 2012; Manji et al., 2001; McEwen \& Magarinos, 2001; Pittenger \& Duman, 2008; Richardson-Jones et al., 2011; Zhuang et al., 1999).

Given the interaction of the 5-HTTLPR $s$ allele and stress on offspring emotionality, behaviours and learning and the effect of experience on structural plasticity (Karg Burmeister, Shedden \& Sen, 2011; Lemaire, Koehl, Moal \& Abrous, 2000; Furlong et al., 1998), we speculate that the enhanced neurogenesis in animals with a genetic inactivation of SERT (compared with HET animals) could enhance their neuroplasticity within the hippocampal circuitry involved in learning and stress responses. Stress has been shown to 
alter neuronal circuitry and connectivity in the amygdala and enhance molecular plasticity in the hippocampus (Djordjevic et al., 2012; Figueiredo, Bodie, Tauchi, Dolgas \& Herman, 2003; Herman, Adams \& Prewitt, 1995; Sripada et al., 2012; Vyas, Jadhav \& Chattarji, 2006). The hippocampus typically exerts inhibitory control over the HPA axis, an effect that is reduced in depression (Duman \& Monteggia, 2006; Jankord \& Herman, 2008; Massart et al., 2012; Sapolsky et al., 1984; Winocur et al., 2006). As this neuroplasticity in HOM animals would be achieved through dysregulated 5-HT systems, it is likely to lead to altered connectivity and activity. This may be a mechanism for the altered stress responses seen in depression (Gold \& Chrousos, 2002), $s$ allele carriers and in SERT compromised rats (Jiang, Wang Luo \& Li, 2009; Miller, Wankerl, Stalder, Kirschbaum \& Alexander, 2013). Thus, animals with enhanced neurogenesis may effectively have an enhanced form of 'learning' in stressful contexts which enhances their stress responses and subsequently enhances the anxiety and depressive response to otherwise less salient stimuli (Heiming et al., 2009).

The enhanced neurogenesis seen in HOMs in the present study are, as discussed above, like due to an increase in extracellular 5-HT levels, leading to an enhanced stimulation of 5-HT receptors. We must, therefore, consider the role of the many different 5-HT receptors in the regulation of neurogenesis in particular. While 5-HT interacts with about 15 different receptors, the 5-HT1A, 5-HT1B/1D, 5-HT2A, 5-HT2C and 5-HT4 receptors have been implicated in neurogenesis (Cryan et al., 2005; Ferrés-Coy et al., 2013; Karabeg et al., 2013; Kondo, Nakamura, Ishida \& Shimada, 2015; Mendez-David et al., 2014).

5-HT1A receptor dysregulation has been implicted in the pathophysiology of depression (Blier \& Ward, 2003; Le François, Czesak, Stebul \& Albert, 2008; Lemonde et al., 2003). The effects of 5-HT1A receptor stimulation is complex as it is highly abundant in the brain, with differential effects of pre- and postsynaptic and auto- and heteroreceptor activation (Celada, Bortolozzi \& Artigas, 2013).

Many studies suggests that hippocampal 5-HT1A heteroreceptor activation is important for increasing neurogenesis and antidepressant effects (Barnes \& Sharp, 1999; Bockaert, Claysen, Bécamel, Dumuis \& Marin, et al., 2006; Pompeiano, Palacios \& Mengod, 1992; Riad et al., 2000; Celada et al., 2013; Haddjeri, Blier \& Montigny, 1998; Hayakawa, Shimizu, Nishida, Motohashi \& Yamawaki, 2008). 5-HT1A heteroreceptors are abundant in limbic and cortical forebrain areas including the hippocampus, amygdala and PFC which are implicated in the regulation of mood and postsynaptic 5-HT1A receptor activation in 
corticolimbic areas is typically antidepressive/anxiolytic (Barnes \& Sharp, 1999; Bockaert et al., 2006; Pompeiano et al., 1992; Riad et al., 2000; Celada et al., 2013; Haddjeri et al., 1998). Moreover, 5-HT1A receptors on granule cells directly contribute to the neurogenic and behavioural response to SSRIs (Samuels et al., 2015). 5-HT1A autoreceptors, in contrast, induce antidepressant effects through their desensitization which increases downstream 5-HT (Artigas, Romero, de Montigny \& Glier, 1996; Blier \& Ward, 2003; Celada et al., 2013; Haddjeri et al., 1998; Hervás et al., 2001). The latter is also likely for 5-HT1B autoreceptors which also desensitize after chronic SSRI treatment (Fabre et al., 2000; Moret \& Briley, 2000).

Thus, 5-HT1A auto- and heteroreceptor stimulation may function to increase neurogenesis via different methods: the effects of increased 5-HT after 5-HT1A autoreceptor desensitization and the activation of downstream neurogenic pathways of 5-HT1A heteroreceptors, such as CREB-mediated neurogenic pathways (Zhang et al., 2016) or astroglial S100 pathways (Amitia, 2001; Azmitia et al., 1995; Whitaker-Azmitia et al., 1990a), respectively. This fits with the finding that HOMs have the highest levels of neurogenesis as increased 5-HT in these animals would increase both 5-HT1A autoreceptor desensitization-mediated and heteroreceptor activated neurogenesis. Indeed, 5-HT1A receptor desensitization occurs in mice (Fabre et al., 2000) and rats (Homberg et al., 2007) lacking SERT. However, this has only been observed in adult rats so far. Whether these receptors are also downregulated at postnatal day 7 remains to be investigated. Neurogenesis may be lower in HETs, as their 5-HT1A autoreceptors may not desensitize since the level of extracellular 5-HT typically in HETs is similar to that of WTs (Kim et al., 2005), this would also lead to less 5-HT1A heteroreceptors activation. HOM animals and humans with two $s$ alleles may thus acquire altered neurocircuitry through 5-HT1A autoreceptor desensitization and increased stimulation of postsynaptic 5-HT1A receptors on granule cells. This is a possible pathway for anxious and depressive outcomes in genetically vulnerable individuals lacking normal SERT levels. In line with this, 5-HT1A autoreceptors have been shown to be involved in anxiety-like behaviours (Gross et al., 2002; Leonardo \& Hen, 2008) and their dysregulation during development results in an anxious and depressive phenotype (GarciaGarcia et al., 2014; Le François et al., 2008; Richardson-Jones et al. 2011).

Individuals with greater extracellular 5-HT since development would also likely undergo increased 5-HT2A receptor stimulation, while non-genetically vulnerable individuals 
who develop depression may have a downregulation of their 5-HT2A receptors later in life which has been observed in depressed individuals (Owens \& Nemroff, 1994). As 5-HT2A receptor activation enhances associative learning and cell proliferation, increased synaptic density and cell survival (Azmitia, 2001, Banasr et al., 2003; Harvey, 2003; Klempin et al., 2010; Niitsu et al., 1995; Vitalis \& Panavelas, 2003), 5-HT2A-stimulated associations may lead to aberrant hippocampal circuitry and contribute to mood dysregulation in HOMs and $s$ allele carriers. As it has been suggested that 5-HT2A receptors are involved in active coping with stress and are associated with enhanced plasticty (Carhart-Harris \& Nutt, 2017), an increase in 5-HT2A receptors later in life may be an adaptive response to stress-induced depression, where increasing learning and neurogenesis could help alleviate depressive symptoms.

Stimulation of 5-HT2C can also enhance neurogenesis in animals (Soumier, Banasr, Goff \& Daszuta, 2010). However, the blockade of 5-HT2C receptors also potentiates SSRIinduced extracellular 5-HT increase (Cremers et al., 2004). Thus, if 5-HT2C receptors were to desensitize after overstimulation in HOM animals this may further increase extracellular 5HT and downstream neurogenesis. Again, we have some (unpublished) evidence for a downregulation of 5-HT2 receptors in HOM rats in adulthood, but comparable results for early in life are still lacking.

As demonstrated previously, increased stimulation of hippocampal 5-HT3 and 5-HT4 receptors may be partly responsible for enhanced neurogenesis in the HOM animals, possibly through increasing p-CREB and/or BDNF for the latter (Kondo et al., 2015; Mendez-David et al., 2014; Pascual-Brazo et al., 2012; Tamburella, Micale, Navarria \& Drago, 2009). 5-HT3 receptors are involved in the regulation of neurodevelopment by 5-HT, e.g. fine-tuning of microcircuitry (Engel et al., 2013). Stimulation of the 5-HT4 receptor may also reverse granule cell maturity like the effects of SSRIs (Imoto et al., 2015; Kobayashi et al., 2010).

Thus, we speculate that overall the increase in 5-HT in HOMs is likely to effect neural activity through various 5-HT receptor pathways.

The current findings inform our understanding of depression especially in the context of the monoamine and neurogenic/neuroplasticity hypotheses. The findings also illustrate that 
maternal SERT genotypes, and their corresponding 5-HT levels may influence the developing offspring.

An increasing number of pregnant women with depression are being treated with SSRIs, this is important as in vitro and in vivo findings with SSRI show that disruptions of 5HT signalling within the placenta can affect feotal cell proliferation and neurodevelopment (Rosenfeld, 2020). SSRIs readily cross the placental barrier (Kim et al., 2005; Lattimore et al., 2005; Mulder, Ververs, de Heus \& Visser, 2011; Noorlander et al., 2008) and feotal exposure to SSRIs can dramatically increase the risk of mortality, long-term alterations in SERT levels in the raphe nucleus and depressive- and anxiety-related behaviours in adult mice which are similar to the behavioural phenotype seen in mice that are genetically deficient in SERT expresssion (Ansorge et al., 2004; Noorlander et al., 2008; Oberlander et al., 2009). Perinatal SSRI treatment and genetic inactivation of SERT both result in developmental delays for rats (Kroeze et al., 2016) while maternal SSRI use and depression in humans is associated with clinically significant emotional and behavioral problems for offspring (Nulman et al., 2012; Oberlander et al., 2010; Pearson et al., 2013). Our findings contribute to our understanding of the consequences of altering maternal and feotal 5-HT on brain plasticity by providing new evidence that maternal 5-HT levels determined by the maternal SERT geneotype affect early offspring neurodevelopment by affecting the level of neurogenesis taking place.

As hippocampal neurogenesis influences learning and memory, the maternal SERT genotype may, through affecting neurogenesis, influence learning and memory performance in offspring. Laplante, Brunet, Schmitz, Ciampi and King (2008) for example, found prenatal maternal stress to be associated with lower intellectual and language performance. WT animals from HET mothers showed a reduction in neurogenesis compared to WT/WT animals, thus, the finding that altered maternal SERT levels affects neurogenesis warrants further investigation of whether and how maternal SERT genotype affects offsping learning capabilities. 
Neurogenesis underlies the therapeutic response to SSRIs (Santarelli et al., 2003) and is therefore though to be therapeutic. If increasing neurogenesis in later life is therapeutic (e.g., after stress-induced depressive episodes) then research into neurogenic pathways is an avenue for treatment research. Nakano-Kobayashi et al. (2017) found that inducing neurogenesis prenatally can rescue aberrant cortical formation in down syndrome mouse embryos and prevent the subsequent development of abnormal behaviours (e.g., decreased exploration, spatial learning, contextual fear learning) in these offspring, as well as rescuing proliferative deficits in human neural stem cells from individuals with down syndrome. SERT KO models may be useful in further studying this effect as the proliferative effects of 5-HT may also facilitate this restorative induction of neurogenesis.

We note that the homogeneity of variance assumption of the ANOVA was not met due to unequal sample sizes, so these results should be interpreted with caution. In addition, some of the groups were relatively small which may have led to a reduction in statistical power. However, the type IV sum of squares analysis used took the unequal variances and missing cells (as WT/HOM or HOM/WT animals are not possible) into account. Moreover the interaction was significant for two regions and all three regions combined and the genotype effect was significant and identical for all three regions, indicating that these results are indeed indicative of real differences and interaction effects of the maternal and offspring genotype.

Another factor to consider is with BrdU labelling as BrdU's bromine side group alters DNA stability increasing the risk of sister chromatid exchanges, mutations, DNA strand breaks and lengthens the cell cycles of the cells it is incorporated within (Taupin, 2007). BrdU is more a marker of DNA synthesis which is used as a proxy signal for the S-phase of the cell cycle and cell proliferation, however, BrdU will also label cells undergoing DNA repair, abortive cell cycle re-entry, as a prelude to apoptosis, and gene duplication without cell division, leading to polyploidy (Taupin, 2007). Thus, BrdU has mitogenic effects and is potentially more toxic to cells than tritiated thymidine (Magavi \& Macklis, 2008; Taupin, 2007). Hancock, Priester, Kidder and Keith (2009), however, did not find this to be the case in vivo. 
Cameron and Mckay (2001) found that commonly used BrdU doses of 50mg/kg only labelled a fraction of $\mathrm{S}$ phase cells in adult rats and the number of labelled cells did not plateau until a dose of $300 \mathrm{mg} / \mathrm{kg}$ was used. Moreover, doses as high as $600 \mathrm{mg} / \mathrm{kg}$ did not show noticeable detrimental effects (Cameron \& Mckay, 2001). Thus, the BrdU dosage used of $25 \mathrm{mg} / \mathrm{kg}$ may not have detected all the newborn cells present. Other neurogenesis detection methods may provide more accurate results. Endogenous cell-cycle proteins, for example, are increasingly being used detect cells during different phases of cell-cycle progression (Kuhn, Eisch, Spalding \& Peterson, 2016). Ki67 is one such marker, expressed both during S phase and through the remainder of the cell cycle (Kuhn et al., 2016).

Maternal care behaviour was not measured in this study but further investigation would serve to examine the contribution (if any) of maternal care on offspring neurogenesis. Alternatively, cross fostering animals immediately after birth could control for genotype changes in maternal care. An important assumption of cross fostering and maternal care studies is that a mother would spend equal time and care for each pup, regardless of its genotype. However, this has not been invesitgated in any detail. Thus, subsequent studies would benefit from rapid (i.e. postnatal day 1) genotyping of the offspring and subsequent analysis of maternal care, for example, using the classical pup retrieval experiment and placing pups from different genotypes in different arms of a maze. This would allow us to invesitgate whether the latency to retrieve pups is dependent on the pup's genotype.

Future studies should further investigate the relationship between 5-HT and neurogenesis in SERT KO rats. This would include investigating whether neurogenesis is also altered in adulthood in HOM and HETs compared to WT. Moreover, while downregulation of 5-HT1A and 5-HT2 receptors have been identified in HOMs in adulthood, it is unclear whether such downregulations already occur early in life (i.e. at postnatal day 7). In addition to the SERTs there are other genetic models for depression, such as the Flinders Sensitive and Fawn Hooded rats (Overstreet, Friedman, Aleksander, Mathé \& Yadid, 2005; Rezvani, Parsian \& Overstreet, 2002). It would be interesting to see whether these animals also demonstrate increased hippocampal neurogenesis early in life.

Given the fact that non-genetic factors are also important in the etiology of depression and, especially early stressful life events, strongly interact with the SERT genotype, future 
research should examine stress-induced changes in serotonergic receptor and neurogenesis in the brains of genetically vulnerable and genetically resilient animals.

Future research should also be directed at investigating the fate of these new cells. It should be examined whether they develop into neurons or glial cells, where in the hippocampal formation they migrate too and ideally, whether they form synaptic contacts with other cells. This could be extended into examining whether experience of stressed or depressed mothers enhances neurogenesis for offspring in a way that serves as adaptive for dealing with future stressors. Any neurogenic or emotional and behavioural differences found between genotypes during early development would need follow up in adulthood to see if they are retained.

The study of neurological diseases/disorders requires the ability to not only determine what molecules are implicated, but their concentrations and their spatial distributions (Schubert et al., 2016; Shariatgorji et al., 2014). Matrix assisted laser desorption ionization is an imaging mass spectrometry technique (MALDI- IMS) that allows for the detection, identification and distribution mapping of hundreds of molecules simultaneously (Hanrieder, Phan, Kurczy \& Ewing, 2013; Shariatgorji et al., 2014). In a next step, MALDI-IMS would be used to identify global brain changes among the SERT-/- genotype, and in particular, changes neurotransmitter quantities and distributions.

\section{Conclusion}

This study examined the effects of offspring and maternal genotype on early postnatal neurogenesis to determine whether early neuroplastic changes occur in SERT knockout rats compared to wild types. We found that offspring genotype increased neurogenesis in a genotype-dependent manner from HET to HOMs but in WT offspring we found that the maternal genotype influenced neurogenesis. WT animals had lower neurogenesis when they had a HET mother while the WT/WT animals showed neurogenesis levels between HET and HOMs. Altogether these results suggest that altered 5-HT during development does indeed affect neuroplasticity in the form of altered levels of neurogenesis which support the notion of a linked monoamine signalling and neurogenic theory of depression. 
CHAPTER TWO

MALDI BRAIN IMAGING 


\section{Introduction}

The study of neurological diseases/disorders requires the ability to not only determine what molecules are implicated, but their concentrations and their spatial distributions. Indeed, the concentrations of chemical compounds/ molecules in the brain are crucial to normal as well as pathological processes, we therefore need this information as well as spatio-temporal distribution information to fully uncover what neurochemical changes occur in disease states (Schubert et al., 2016; Shariatgorji et al., 2014).

Matrix assisted laser desorption ionization imaging mass spectrometry allows for the detection, identification and distribution mapping of hundreds of molecules simultaneously without a great deal of a priori knowledge of potential targets of interest (Hanrieder et al., 2013; Shariatgorji et al., 2014). This mass spectrometry technique involves sample analytes being embedded in a matrix that absorbs energy from an ultraviolet laser leading to the desorption and ionization of the constituent chemicals and the acquisition of a mass spectra of these chemicals (Hanrieder et al., 2013; Schubert et al., 2016). Thus, this technique allows for the identification of compounds that are dysregulated between samples.

\section{MALDI principles}

MALDI-IMS is an imaging mass spectrometry technique effective for molecular studies of complex biological samples, such as tissue sections, directly (Schwamborn \& Caprioli, 2010; Setou, 2010). MALDI-IMS is theoretically capable of analysing high molecular mass biomolecules of $200 \mathrm{kDa}$ or more, as well as small molecules of less than 1 kDa molecular mass (Chaurand, Cornett \& Caprioli., 2006; Cornett Reyzer, Chaurand \& Caprioli, 2007; Karas, Bahr, Ingendoh \& Hillenkamp, 1989; Schwamborn \& Caprioli, 2010; Tanaka et al., 1988). MALDI-IMS allows for the identification of not only complex normal biological processes, but also pathological processes through the direct detection of biomolecules such as proteins, peptides, lipids as well as their spatial distribution and intensity profiles within tissues and single cells (Cornett et al., 2007; Hanrieder et al., 2013; Zimmerman, Monroe, Tucker, Rubakhin \& Sweedler, 2008). Essentially, mass spectrometry measures the masses of isolated gas-phase ionised molecules directly by their behaviour in electromagnetic fields (Beavis \& Chait, 1990) while the topical addition of an organic matrix over the sample before laser irradiation assists in laser ablation of the molecules and lessens 
fragmentation to stabilise molecules (Medzihradszky et al., 2000; Zenobi \& Knockenmuss, 1998; Zimmerman et al., 2008). See figure 13.

Figure 13

Simplified MALDI-IMS workflow

Tissue sectioning
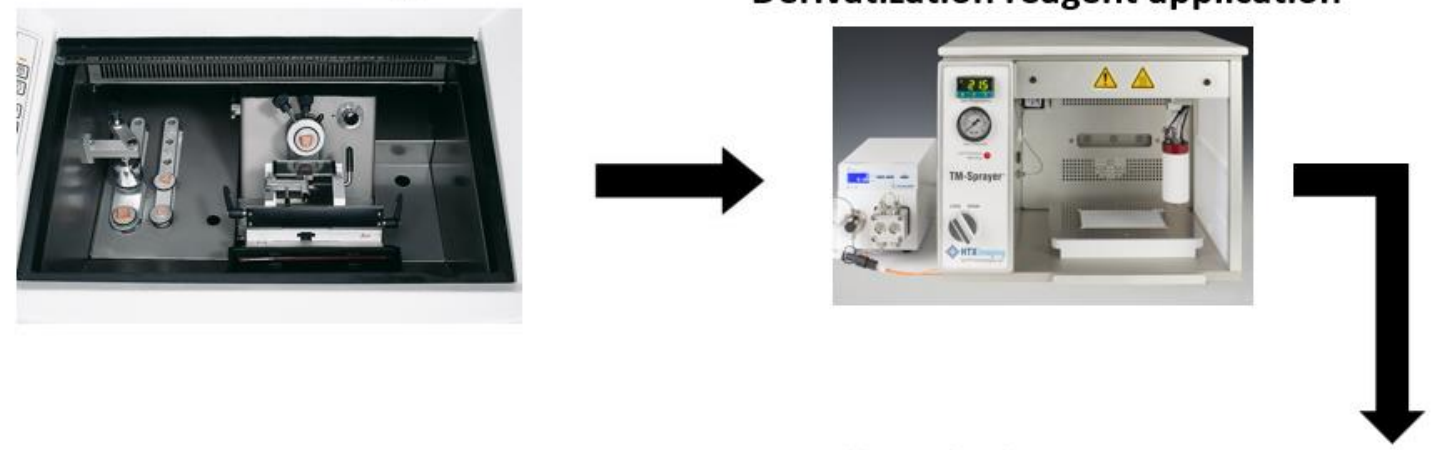

Derivatization reagent application

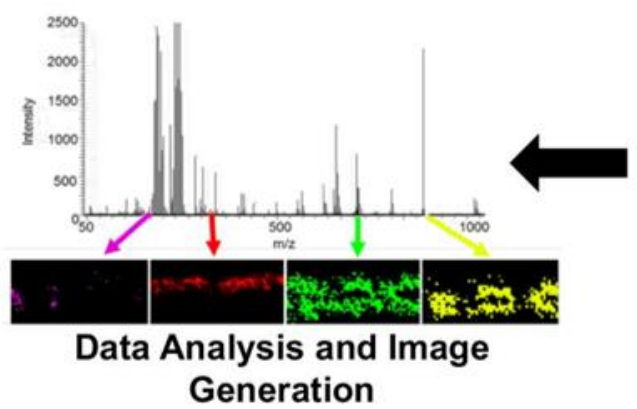

Matrix application
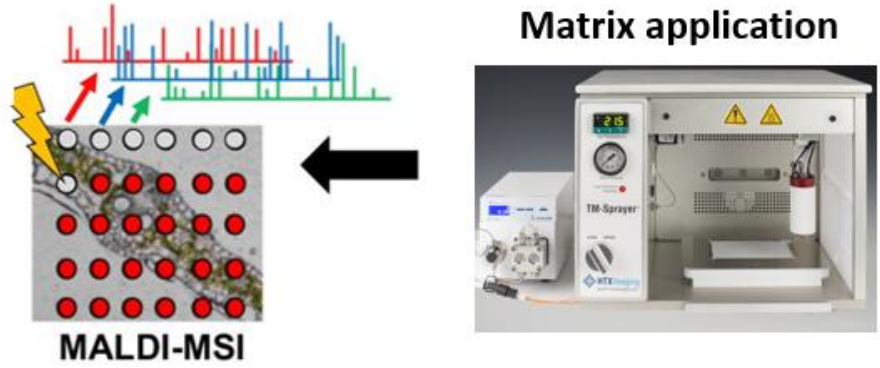

Note. Modified from Dueñas, Larson and Lee (2019).

Thus, MALDI-IMS generates two-dimensional ion images for each of the mass-to charge $(\mathrm{m} / \mathrm{z})$ ratio values detected at each pixel (microscopic area) of tissue and therefore provides the spatial distribution the constituent compounds within that sample (Caprioli, Farmer \& Gile, 1997; Chaurand, Schwartz \& Caprioli, 2002) MALDI is also amenable to a broad range of analytes including proteins, peptides, cell metabolites, lipids, hormones, glycans, toxins, drugs and drug metabolites (Aichler \& Walch, 2015).

Chemical stains, immunohistochemical tags and radiolabels are common methods for visualizing and identifying molecular targets. However, they are limited in their specificity and the number of target compounds which can be simultaneously investigated. Moreover, use of specific molecular tags requires a priori knowledge of the target species, which limits their use for molecular discovery (Cornett et al., 2007; Hanrieder et al., 2013). MALDI-IMS 
does not require a priori knowledge of target species nor is it dependent on antibody or primer availability and specificity which allows for identification of new compounds (Hanreider et al., 2013; Schubert et al., 2016).

Classical proteomic, metabolomic and lipidomic techniques involve homogenization steps and therefore cannot provide spatial distribution information (Schubert et al., 2016), unless repeated over many different brain regions. Sample preparation for these techniques also involve multiple time-consuming steps that can lead to analyte material loss while MALDI-IMS involves minimal sample preparation and sample handling (Schwartz, Reyzer \& Caprioli, 2003; Setou, 2010). Thus, MALDI's main advantages include detection, identification and spatial mapping of hundreds of compounds simultaneously without a priori knowledge of targets (Caprioli et al., 1997; Cornett et al., 2007; Hanrieder et al., 2013; Schubert et al., 2016; Schwartz et al., 2003; Setou, 2010). Additionally, MALDI-IMS is also advantageous as it offers high tolerance against contaminants and buffers (Calvano, Monopoli, Cataldi \& Palmisano, 2018; Yamaguchi, Fujita, Fujino \& Korenaga, 2008), its high sensitivity and compatibility with diverse mass analysers (Palanisamy, Huang, Zhao, Zhu \& Zhang, 2019).

\section{MALDI-IMS workflow}

\section{Derivatization}

Small molecules such as neurotransmitters which we are interested in, tend to have poor ionization efficiencies, and can be masked by the overlapping signals of isobaric compounds (Esteve, Tolner, Shyti, van den Maagdenberg \& McDonnell, 2016; Guo \& He, 2007; Hanrieder et al., 2013; Shariatgorji et al., 2014; Ye, Wang, Greer, Strupat \& Li, 2013). Chemical derivatization improves the ionization and detection of small molecules, makes them less volatile and increases their mass by a known amount (Chacon et al., 2011; Esteve et al., 2016; Zaikin \& Halket, 2006). Derivatization typically introduces an ionizable or ionic group to the analyte being derivatized (Zaikin \& Halket, 2006). Shariatgorji and colleagues (2014, 2015) developed a method for chemical derivatization of smaller molecules such as neurotransmitters by reaction with pyrylium salts that facilitates their detection by MALDIIMS. Pyrylium salts such as 2,4-diphenyl-pyranylium tetrafluoroborate (DPP TFB) react with the amino group (NH2) of amines to convert the amine into a pyrylium salt structure and 
produce N-alkyl- or N-aryl- pyridinium derivatives (Shariatgorji et al., 2014, 2015).

However, there are other reagents that can be used to derivatize amine metabolites in tissue (e.g. p-N,N,Ntrimethylammonioanilyl N-hydroxysuccinimidyl carbamate iodide [TAHS] and 4-hydroxy-3-methoxycinnamaldehyde [CA]) each with its own optimal reaction temperature and concentration (Esteve et al., 2016; Manier, Spraggins, Reyzer, Norris \& Caprioli, 2014).

\section{Matrix application}

The defining step of MALDI-IMS process involves coating the sample with an energy absorbing matrix that facilitates ablation and ionization of the sample compounds Caprioli et al., 1997; Ehring et al., 1992; Hanrieder et al., 2013). The matrix facilitates soft desorption of analyte molecules upon laser irradiation, by absorbing some of the laser's energy, and reduces analyte fragmentation making them more stable molecules during analysis (Caprioli et al., 1997; Hillenkamp, Karas, Beavis \& Chait, 1991; Karas, Bachmann, Bahr \& Hillenkamp, 1987; Medzihradszky et al., 2000; Tanaka et al., 1988; Zaikin \& Halket, 2006). The matrix usually consists of small organic aromatic molecules with high absorbance at the wavelength of the laser used, it is typically a crystalline UV absorbing matrix, although other matrices can be used which differ in the amount of energy they impart to the sample biomolecules during desorption and ionisation and the resulting degree of molecule fragmentation (Calvano et al., 2018; Leopold, Popkova, Engel \& Schiller, 2018; Mann, Hendrickson \& Pandey, 2001; Wang, Zhao, Guo, Cai \& Liu 2013).

MALDI-IMS requires that analytes within tissue samples be embedded within the crystallised matrix or they may delocalise (Zimmerman et al., 2008). Typically, the analyte is co-crystallized with a surrounding excess of the solid matrix that crystallizes as it dries, essentially embedding these analytes within matrix crystals (Calvano et al., 2018; Dreisewerd, 2003; Leopold et al., 2018; Zenobi \& Knockenmuss, 1998). The matrix typically consists of a solution of water, organic solvents containing ethanol/methanol or acetonitrile as well as a strong acid such as trifluroacetic acid (TFA) in which the matrix is dissolved (Palanisamy et al., 2019; Schwartz et al., 2003).

The choice of matrix used determines which molecules can be analysed and the level of sensitivity in their detection (Esteve et al., 2016). Similarly, the matrix deposition 
conditions, composition, concentration, application method and thickness of coating are all factors contributing to the quality of the mass spectra attained (Zimmerman et al., 2008). An appropriate matrix should result in a high signal-to-noise $(\mathrm{S} / \mathrm{N})$ ratio, high spectra resolution, little analyte fragmentation and moderate matrix background to minimize the interference between matrix and analyte signals, although this is challenging for small molecules (Leopold et al., 2018). Suitable matrices must fulfil the five following criteria (Leopold et al., 2018). First, matrices require strong absorption of the wavelength of the laser as ionization efficiency and ion yield effectively increase with an increasing absorption coefficient of the matrix. Second, matrices should ensure analyte ion formation. In cinnamic and benzoic acid derivatives the carboxylic acid structure being polar and acidic ensures matrix solubility in polar solvents and analyte protonation (Leopold et al., 2018). Third, the matrix needs to remain stable under high vacuum conditions. Fourth, ideal matrices should isolate the ions generated and prevent the formation of analyte clusters such as dimer formations; this is a primary reason for the large excess of matrix over the analyte. Lastly, crystals should be as homogenous as possible to ensure high 'shot-to-shot' reproducibility (Leopold et al., 2018).

There are three classes of matrices: classic organic matrices such as cinnamic acid or benzoic acid derivatives which are most commonly used, liquid crystalline matrices for even softer ionization and inorganic matrices such as graphite (Esteve et al., 2016; Lemaire et al., 2006; Leopold et al., 2018). Cinnamic acid derivatives have an advantage of having high tolerance of high concentrations of ionic contaminants such as salts and lipids (Beavis \& Chait, 1990). Other desorption and ionization techniques, such as fast atom bombardment and electrospray atmospheric pressure ionization, can get saturated with these contaminating signals (Beavis \& Chait, 1990).

The most commonly used organic matrices are $\alpha$-cyano- 4 hydroxycinnamic acid (CHCA), 2,5-dihydroxybenzoic acid (DHB) and Sinapinic acid (SA), with the first two used for lower weight analyte species and the latter peforming better for higher mass analytes (Norris \& Caprioli, 2016; Schwartz et al., 2003; Zimmerman et al., 2008). CHCA crystals are smaller than those of DHB (Altelaar et al., 2007). CHCA generally results in high sensitivity and is a commonly used matrix for smaller low weight (LW) compounds and produces peptide signals substantially higher than other cinnamic acid derivatives indicating more efficient ionization (Beavis \& Chait, 1992; Lidgard \& Duncan, 1995; Mann et al., 2001; Schwartz et al., 2003; Zimmerman et al., 2008). 
Matrix deposition can be either as individual droplets (spotted) on specific areas of interest on the sample tissue, or as a homogenous layer (spray coated) over the entire tissue depending on the spatial resolution desired (Chaurand et al., 2006; Chaurand, Norris, Cornett, Mobley \& Caprioli 2006a; Norris \& Caprioli, 2013; Schwartz et al., 2003). Spotting allows for faster analysis but tends to produce more irregular crystals and lower resolution profiles of the signals in the chosen sample regions (Schwartz et al., 2003). Spray coating is done either via pneumatic-assisted or electrospray deposition where controlled amounts of matrix dissolved in organic solvents (to achieve stable spraying conditions, e.g., ethanol. acetone, acetonitrile) are sprayed homogenously on the sample tissue in multiple layers (Schwartz et al., 2003). The advantage of automatic spraying is that the optimized spraying conditions can be replicated and applied to large sample sets to ensure reproducibility (Norris \& Caprioli, 2016). Spray coating allows for mass spectra to be acquired for the entirety of the sample; therefore, it produces higher resolution images (Schwartz et al., 2003).

Spatial resolution increases as matrix crystal size decreases (Li et al., 2016). Crystal size can be reduced by using a higher solvent percentage (Norris \& Caprioli, 2016). The matrix solvent solution evaporates leaving the matrix crystallized on the sample tissue. Matrix concentration affects the crystal coverage and ultimately the mass spectral quality (Schwartz et al., 2003). Higher matrix concentrations result in greater crystal coverage over the sample and higher quality spectra (Lemaire et al., 2006; Schwartz et al., 2003). Schwartz et al. (2003) found that 50:50 ratios of solvents to water produced the best quality spectra, however, this ratio typically requires optimization.

\section{Laser irradiation}

A UV-absorbing wavelength laser (there are several UV laser wavelengths and other lasers such as nitrogen and infrared; Zimmerman et al., 2008) then scans across the sample leading to molecules (analytes) to desorb from the sample as multiple laser pulses resonantly excite the matrix, causing rapid localized heating and subsequent co-ablation of analyte and matrix molecules (Dreisewerd, 2003; Hanrieder et al., 2013; Karas \& Kruger, 2003; Schubert et al., 2016; Zaikin \& Halket, 2006; Zimmerman et al., 2008).

\section{Ionization}


Upon laser irradiation the matrix vaporizes and carries with it the co-crystallised analyte molecules into the vapor/ gas phase (Leopold et al., 2018). Ionization occurs through this desorption, where the energy applied from the laser directly produces gaseous ions from the solid- (or liquid-) state sample (Dreisewerd, Schürenberg, Karas \& Hillenkamp, 1995; Wang et al., 2013; Zimmerman et al., 2008). In the gas phase, ions are exchanged between the matrix and analyte allowing the formation of analyte ions (Leopold et al., 2018).

Soft ionisation techniques such as MALDI impart low internal/residual energy during the ion formation process resulting in less fragmentation of analyte molecules than hard ionisation and yields more or less direct measures of molecular weights of the molecules of interest via conversion to singly or multiply protonated [Molecular mass $+\mathrm{H}$ ] + or deprotonated ions [Molecular mass - H]- that are positively or negatively charged respectively (Medzihradszky et al., 2000; Yamaguchi et al., 2008). However, analyte molecules are typically singly protonated forming quasi-molecular ions with molecular weights corresponding to the original analyte molecular weight plus (or minus in the case of negatively charged ions) the mass of the ion required to produce a charge (Calvano et al., 2018; Caprioli et al., 1997; Leopold et al., 2018; Wang et al., 2013; Zimmerman et al., 2008).

\section{Detection}

The desorped ions are then detected by time-of-flight (TOF) analysers which are the most common type of mass analyser and are typically used for detecting neurotransmitters (Palanisamy et al., 2019). The mass of an ion is determined using its velocity, this involves accelerating the ions in an electrical field (typically 20,000V; Hillenkamp et al., 1991; Leopold et al., 2018). After each laser pulse the resulting newly formed ions pass through a charged grid to accelerate them to a fixed amount of kinetic energy, these ions then travel freely over a field-free drift zone down a flight tube where separation based on their $\mathrm{m} / \mathrm{z}$ ratio occurs (Hillenkamp et al., 1991; Leopold et al., 2018; Mann et al., 2001). As the acceleration energy is constant, the principle of ion mobility dictates that ions with a smaller mass will travel faster than ions with a larger mass and thus are detected before larger ions, this produces a TOF spectrum of peaks at different times corresponding to the density of a molecule at a particular mass (Chaurand et al., 2002; Mann et al., 2001; Zimmerman et al., 2008). Ion density images can then be produced for each detected molecule (Chaurand et al. 
2002). The mass spectrometer determines the ions' molecular weights, thereby allowing their identification as well as their spatial localisation within the tissue.

The data array consists of a grid of laser spots, where each spot or pixel is a discrete area of tissue (typically 50x50microns), irradiated by the laser, which contains an entire mass spectrum of the ion masses detected at that discrete location and their densities (Caprioli et al., 1997; Chaurand et al., 2006, 2006a; Cornett et al., 2007). Thus, specific m/z values correspond to specific molecular weights. Resulting mass spectra typically yield 300-1000 signals of varying intensity each (Chaurand et al., 2002).

Currently laser beam width and matrix characteristics (e.g., size of droplets and crystals) limit spatial resolution to $10-20 \mu \mathrm{m}$ with most MALDI-TOF mass spectrometers using laser spot diameters of $50 \mu \mathrm{m}$ - this represents the pixel size of the ion image (Chaurand et al., 2006, 2006a; Cornett et al., 2007; Hanrieder et al., 2013; Schwamborn \& Caprioli, 2010; Zimmerman et al., 2008).

Coupling MALDI with TOF-MS allows for a virtually unlimited mass range to be monitored (Wang et al., 2013), however, the mass range below 500Da is often obscured by matrix ions (Mann et el., 2001). MALDI-IMS also suffers from reduced sensitivity and compromised mass accuracy when resolving proteins at the higher molecular mass range with some researchers suggesting compromised accuracy at masses as low as $40 \mathrm{kDa}$ (Beavis \& Chait, 1990; Schwartz et al., 2003). Proteins tend to fragment during MALDI-IMS which results in a broad range of peaks and a decrease in sensitivity, therefore, MALDI-IMS is mostly applied to the analysis of peptides (Mann et al., 2001). 


\section{Method}

\section{Animals}

All animals were housed in the Victoria University of Wellington vivarium and in accordance with the Animal Welfare Act 1999, with all procedures being pre-approved by the VUW Animal Ethics Committee (AEC number 22375). A total of 9 adult (PND80, WT $n=4$, $\operatorname{HOM} n=5)$ and 10 adolescents (PND21, WT $n=4, \operatorname{HOM} n=6)$ male Wistars were used in this study, all bred from HET male - HET female pairings.

All animals were housed in polycarbonate cages with one dam and her offspring per cage in a temperature and humidity-controlled environment at $21^{\circ} \mathrm{C} \pm 2{ }^{\circ} \mathrm{C}$ and $55-60 \%$ respectively. A reverse 12-hour light cycle was used with lights on at 1900 - 0700 hours. Food and water were available to the animals ad libitum. All subjects were weaned at PND 21 from mothers. Tissue samples from an ear clipping were sent to be genotyped on PND 21 (Transnetyx, Cordova US).

\section{Tissue preparation}

After conscious decapitation (in order to prevent hypoxia-induced tissue degradation and prevent neurochemical alterations caused by general anaesthetics), brain tissue from adult male Wistar rats was rapidly extracted covered in foil and snap frozen within 45 seconds using liquid nitrogen to prevent degradation of neurotransmitters.

Samples were transferred to a cryostat (Lecia CM3050) at $-20^{\circ} \mathrm{C}$ for adult brains and $21^{\circ} \mathrm{C}$ to $-22^{\circ} \mathrm{C}$ for adolescent brains and allowed to acclimatise for a minimum of 60 minutes to improve sectioning quality. Following acclimatization, sections were halved down the longitudinal fissure with a cryomicrotome blade and subsequently the right hemisphere was mounted on a sample plate using OCT (optimal cutting temperature polymer) to attach the lateral side of the brain to the sample plate without any OCT surrounding the area where sections were to be taken from.

Sections were first trimmed to create an even cutting surface, following which, sagittal sections were obtained at $20 \mu \mathrm{m}$ thickness. Sectioned were carefully placed on cooled MALDI target slides which were also kept at $-20^{\circ} \mathrm{C}$ in the cryomicrotome using a thin 
(sparsely bristled, synthetic bristles are less likely to retain melted tissue) paintbrush and was then thaw-mounted by placing a gloved hand briefly under the slide, resulting in rapid tissue adherence to the sample slide. This also avoids residual ice crystal formation and the loss of water-soluble compounds associated with gradual adherence. Slides were stored in a $-80^{\circ} \mathrm{C}$ freezer until they were to be imaged.

\section{Materials}

The chemicals 2-4-6-Triphenylpyrylium Tetrafluroborate (TPP), 2,4,6trimethylpyrylium tetrafluoroborate (TMP), 4-hydroxy-3-methoxycinnamaldehyde (CA), 1,1'-thiocarbonyldiimidazole (TCDI), Triethylamine (TEA), $\alpha$-cyano-4 hydroxycinnamic acid (CHCA) and N,N-Diisopropylethylamine (DIPEA) were purchased from Sigma Aldrich suppliers in Auckland, New Zealand.

\section{Procedure}

Before MALDI-IMS analysis, slides were dried at room temperature for 30 minutes using a vacuum desiccator to prevent condensation forming and delocalisation of compounds. After acquiring a scan of the slice, the slide was subjected to chemical derivatization followed by matrix deposition prior to MALDI-IMS analysis. The methods for each of derivatization reagents tested are described below.

To validate the accurate alignment of the spectra, reference points were added using a $1 \mathrm{mg} / \mathrm{ml}$ spot of dopamine (Dopamine hydrochloride; Sigma-Aldrich) dissolved in distilled water), and $1 \mathrm{mg} / \mathrm{ml}$ of serotonin (Serotonin hydrochloride; Sigma-Aldrich) dissolved in distilled water), spotted near the tissue.

The following derivatization reagents were selected optimized for their abilities to derivatize LW molecules including neurotransmitters: TPP, TMP, TCDI, CA (Chacon et al., 2011; Dueñas et al., 2019; Esteve et al., 2016; Manier et al., 2014; Palanisamy et al., 2019; Shariatgorji et al., 2014, 2015). 
To test the ability of TPP to derivatise low weight neurotransmitters, $1.8 \mathrm{mg} / \mathrm{ml}$ of TPP was diluted in a $70 \%$ methanol and $0.5 \%$ Triethylamine solution. This solution was evenly sprayer over the slide with a flow rate of $0.07 / \mathrm{ml}$ per minute, $600 \mathrm{~mm} / \mathrm{min}, 8$ passes, at $150 \mathrm{PSI}$ and $50^{\circ} \mathrm{C}$ using an automatic sprayer (HTX TM-Sprayer). TPP was initially selected due to its ability to react with amines creating pyrylium salt structures. This derivatization reaction occurs at ambient temperature and pressure with no need for heat, stirring, agitation or extensive incubation, which helps to preserve the localization of endogenous molecular compounds in the sample (Shariatgorji et al., 2014, 2015).

The sample slide was incubated in an empty petri dish and placed in a dark cupboard for 2 hours. During this incubation phase, the derivatisation agent reacts with the amines through creating a basic environment, opening the chemical structure, and allowing for the formation of the pyrylium salt structure. Due to the acidic nature of the matrix, there was no need to re-acidify the sample as seen in prior studies (Kamath, Diedrich, \& Hindsgaul, 1996), as this was likely to increase delocalisation.

Finally, $\alpha$-cyano-4 hydroxycinnamic acid (CHCA, Sigma-Aldrich, $8 \mathrm{mg} / \mathrm{ml}$ in a solution of $70 \%$ acetonitrile and $0.2 \%$ Trifloroacetic acid) was used as the matrix due its broad use in soft ionisation techniques. $2 \mathrm{~mL}$ of the matrix solution was applied using the automatic sprayer $\left(75^{\circ} \mathrm{C}\right.$, flow rate $0.07 / \mathrm{ml}$ per minute, velocity $600 \mathrm{~mm} / \mathrm{min}, 8$ passes, 150 PSI) with the TPP acting as a proton donor allowing for the absorption of the laser resulting in the ionization and abolition of the sample tissue. Finally, the sample was incubated with $4 \mathrm{ml}$ of $50 \%$ methanol solution and $0.2 \mathrm{ml}$ of acetic acid.

Following CHCA application and incubation the sample slide was loaded into the mass spectrometer (AB Sciex TOF/TOF 5800), which uses a Nd:YLF (neodymium-doped yttrium lithium fluoride) variable laser set at $349 \mathrm{~nm}$ in a vacuum chamber to process the spectra using the time-of-flight principle. The laser ablates and ionises the sample with a resolution of $50 \mu \mathrm{m}^{2}$ per pixel. Following the completion of the MALDI procedure, the output spectra were analyses using Bitmap software.

\section{Derivatization method 2) TPP with DIPEA}

Next, TPP was tested using the base DIPEA instead of TEA. 
$1.5 \mathrm{mg} / \mathrm{ml}$ of TPP was diluted in a $100 \%$ methanol and DIPEA solution $(0.658 \mu \mathrm{L}$ DIPEA per $1.5 \mathrm{mg} / \mathrm{mL}$ TPP). This solution was evenly sprayed over the slide (flow rate $0.07 / \mathrm{ml}$ per minute, $600 \mathrm{~mm} / \mathrm{min}, 8$ passes, $150 \mathrm{PSI}, 50^{\circ} \mathrm{C}$ ). After spraying, the slide was incubated for two hours in a covered petri dish inside a dark cupboard. After this, $2 \mathrm{~mL}$ of CHCA $(8 \mathrm{mg} / \mathrm{ml}$ in a solution of $70 \% \mathrm{ACN}$ and $0.2 \%$ TFA) was sprayed over the slide (flow rate $0.07 / \mathrm{ml}$ per minute, velocity $600 \mathrm{~mm} / \mathrm{min}, 8$ passes, $150 \mathrm{PSI}, 75^{\circ} \mathrm{C}$ ). The slide was incubated again for two hours in a covered dish placed in a dark cupboard. Following incubation, the slide was loaded into the mass spectrometer to obtain the mass spectra. 


\section{Initial results}

The ion images we obtained showed that our initial TPP derivatization method was not successfully derivatizing neurotransmitters. We observed little to no detection of neurotransmitters. Initially we thought this may be due to the TEA used being old as it had turned yellowish in colour. We next used the base DIPEA instead of TEA with TPP however we came across the same issue of little to no detection. While we did not have enough time to troubleshoot this problem further, the next steps are to try different derivatization reagents: TMP, TCDI, CA. 


\section{References}

Abrous, D. N., Koehl, M., \& Le Moal, M. (2005). Adult Neurogenesis: From Precursors to Network and Physiology. Physiological Reviews, 85(2), 523-569. https://doi.org/10.1152/physrev.00055.2003

Aichler, M., \& Walch, A. (2015). MALDI Imaging mass spectrometry: Current frontiers and perspectives in pathology research and practice. Laboratory Investigation, 95(4), 422-431. https://doi.org/10.1038/labinvest.2014.156

Airan, R. D., Meltzer, L. A., Roy, M., Gong, Y., Chen, H., \& Deisseroth, K. (2007). High-Speed Imaging Reveals Neurophysiological Links to Behavior in an Animal Model of Depression. Science, 317(5839), 819-823. https://doi.org/10.1126/science.1144400

Aitken, A. R., \& Törk, I. (1988). Early development of serotonin-containing neurons and pathways as seen in wholemount preparations of the fetal rat brain. Journal of Comparative Neurology, 274(1), 32-47. https://doi.org/10.1002/cne.902740105

Albert, P. R., \& Lemonde, S. (2004). 5-HT1A Receptors, Gene Repression, and Depression: Guilt by Association. The Neuroscientist, 10(6), 575-593.

https://doi.org/10.1177/1073858404267382

Alenina, N., \& Klempin, F. (2015). The role of serotonin in adult hippocampal neurogenesis. Behavioural Brain Research, 277, 49-57. https://doi.org/10.1016/j.bbr.2014.07.038

Almeida, O. P., Alfonso, H., Hankey, G. J., \& Flicker, L. (2010). Depression, antidepressant use and mortality in later life: The Health In Men Study. PloS One, 5(6), e11266. https://doi.org/10.1371/journal.pone.0011266

Altelaar, A. F. M., Taban, I. M., McDonnell, L. A., Verhaert, P. D. E. M., de Lange, R. P. J., Adan, R. A. H., Mooi, W. J., Heeren, R. M. A., \& Piersma, S. R. (2007). High-resolution MALDI imaging mass spectrometry allows localization of peptide distributions at cellular length scales in pituitary tissue sections. International Journal of Mass Spectrometry, 260(2), 203-211. https://doi.org/10.1016/i.ijms.2006.09.028

Altman, J., \& Das, G. D. (1966). Autoradiographic and histological studies of postnatal neurogenesis. I. A longitudinal investigation of the kinetics, migration and transformation of cells incoorporating tritiated thymidine in neonate rats, with special reference to postnatal 
neurogenesis in some brain regions. Journal of Comparative Neurology, 126(3), 337-389. https://doi.org/10.1002/cne.901260302

Alvarez-Buylla, A., \& García-Verdugo, J. M. (2002). Neurogenesis in Adult Subventricular Zone. Journal of Neuroscience, 22(3), 629-634. https://doi.org/10.1523/JNEUROSCI.22-03$\underline{00629.2002}$

Amaral, D. G., Scharfman, H. E., \& Lavenex, P. (2007). The dentate gyrus: Fundamental neuroanatomical organization (dentate gyrus for dummies). Progress in Brain Research, 163, 3-22. https://doi.org/10.1016/S0079-6123(07)63001-5

American Psychiatric Association. (2013). Diagnostic and Statistical Manual of Mental Disorders (DSM-5®). American Psychiatric Pub.

Andersen, P., Soleng, A. F., \& Raastad, M. (2000). The hippocampal lamella hypothesis revisited11Published on the World Wide Web on 12 October 2000. Brain Research, 886(1), 165-171. https://doi.org/10.1016/S0006-8993(00)02991-7

Ansorge, M. S., Hen, R., \& Gingrich, J. A. (2007). Neurodevelopmental origins of depressive disorders. Current Opinion in Pharmacology, 7(1), 8-17. https://doi.org/10.1016/j.coph.2006.11.006

Ansorge, M. S., Morelli, E., \& Gingrich, J. A. (2008). Inhibition of Serotonin But Not Norepinephrine Transport during Development Produces Delayed, Persistent Perturbations of Emotional Behaviors in Mice. Journal of Neuroscience, 28(1), 199-207.

https://doi.org/10.1523/JNEUROSCI.3973-07.2008

Ansorge, M. S., Zhou, M., Lira, A., Hen, R., \& Gingrich, J. A. (2004). Early-Life Blockade of the 5-HT Transporter Alters Emotional Behavior in Adult Mice. Science, 306(5697), 879-881. https://doi.org/10.1126/science.1101678

Arnold, S. E., \& Trojanowski, J. Q. (1996). Human fetal hippocampal development: I. Cytoarchitecture, myeloarchitecture, and neuronal morphologic features. Journal of Comparative Neurology, 367(2), 274-292. https://doi.org/10.1002/(SICI)10969861(19960401)367:2<274::AID-CNE9>3.0.CO;2-2

Artigas, F., Romero, L., de Montigny, C., \& Blier, P. (1996). Acceleration of the effect of selected antidepressant drugs in major depression by 5-HT1A antagonists. Trends in Neurosciences, 19(9), 378-383. https://doi.org/10.1016/S0166-2236(96)10037-0 
Austin, M.-P., Mitchell, P., \& Goodwin, G. M. (2001). Cognitive deficits in depression: Possible implications for functional neuropathology. The British Journal of Psychiatry, 178(3), 200206. https://doi.org/10.1192/bjp.178.3.200

Azmitia, E.C., Dolan, K., \& Whitaker-Azmitia, P. M. (1990). S-100B but not NGF, EGF, insulin or calmodulin is a CNS serotonergic growth factor. Brain Research, 516(2), 354-356. https://doi.org/10.1016/0006-8993(90)90942-5

Azmitia, E. C. (2001). Modern views on an ancient chemical: Serotonin effects on cell proliferation, maturation, and apoptosis. Serotonin: From the Molecule to the Clinic, 56(5), 413-424. https://doi.org/10.1016/S0361-9230(01)00614-1

Azmitia, E. C., Gannon, P. J., Kheck, N. M., \& Whitaker-Azmitia, P. M. (1996). Cellular Localization of the 5-HT1A Receptor in Primate Brain Neurons and Glial Cells. Neuropsychopharmacology, 14(1), 35-46. https://doi.org/10.1016/S0893-133X(96)80057-1

Azmitia, E. C., Rubinstein, V. J., Strafaci, J. A., Rios, J. C., \& Whitaker-Azmitia, P. M. (1995). 5HT1A agonist and dexamethasone reversal of para-chloroamphetamine induced loss of MAP2 and synaptophysin immunoreactivity in adult rat brain. Brain Research, 677(2), 181-192. https://doi.org/10.1016/0006-8993(95)00051-Q

Azmitia, E. C., \& Segal, M. (1978). An autoradiographic analysis of the differential ascending projections of the dorsal and median raphe nuclei in the rat. Journal of Comparative Neurology, 179(3), 641-667. https://doi.org/10.1002/cne.901790311

Bakermans-Kranenburg, M. J., \& van IJzendoorn, M. H. (2008). Oxytocin receptor (OXTR) and serotonin transporter (5-HTT) genes associated with observed parenting. Social Cognitive and Affective Neuroscience, 3(2), 128-134. https://doi.org/10.1093/scan/nsn004

Banasr, M., Hery, M., Printemps, R., \& Daszuta, A. (2004). Serotonin-Induced Increases in Adult Cell Proliferation and Neurogenesis are Mediated Through Different and Common 5-HT Receptor Subtypes in the Dentate Gyrus and the Subventricular Zone. Neuropsychopharmacology, 29(3), 450-460. https://doi.org/10.1038/sj.npp.1300320

Barlow, C., \& Targum, S. D. (2007). Hippocampal Neurogenesis: Can it be a Marker for New Antidepressants? Psychiatry (Edgmont), 4(5), 18-20.

Barnes, N. M., \& Sharp, T. (1999). A review of central 5-HT receptors and their function. Neuropharmacology, 38(8), 1083-1152. https://doi.org/10.1016/S0028-3908(99)00010-6 
Bath, K. G., Akins, M. R., \& Lee, F. S. (2012). BDNF control of adult SVZ neurogenesis. Developmental Psychobiology, 54(6), 578-589. https://doi.org/10.1002/dev.20546

Bauer, P. J. (2004). Getting explicit memory off the ground: Steps toward construction of a neurodevelopmental account of changes in the first two years of life. Developmental Review, 24(4), 347-373. https://doi.org/10.1016/j.dr.2004.08.003

Bayer, S. A. (1980). Development of the hippocampal region in the rat II. Morphogenesis during embryonic and early postnatal life. Journal of Comparative Neurology, 190(1), 115-134. https://doi.org/10.1002/cne.901900108

Bearer, E. L., Zhang, X., Janvelyan, D., Boulat, B., \& Jacobs, R. E. (2009). Reward circuitry is perturbed in the absence of the serotonin transporter. NeuroImage, 46(4), 1091-1104. https://doi.org/10.1016/j.neuroimage.2009.03.026

Beavis, R. C., \& Chait, B. T. (1990). Rapid, sensitive analysis of protein mixtures by mass spectrometry. Proceedings of the National Academy of Sciences, 87(17), 6873-6877.

Becker, S., MacQueen, G., \& Wojtowicz, J. M. (2009). Computational modeling and empirical studies of hippocampal neurogenesis-dependent memory: Effects of interference, stress and depression. Brain Research, 1299, 45-54. https://doi.org/10.1016/j.brainres.2009.07.095

Belmaker, R. H., \& Agam, G. (2008). Major Depressive Disorder. New England Journal of Medicine, 358(1), 55-68. https://doi.org/10.1056/NEJMra073096

Benekareddy, M., Goodfellow, N. M., Lambe, E. K., \& Vaidya, V. A. (2010). Enhanced Function of Prefrontal Serotonin 5-HT2 Receptors in a Rat Model of Psychiatric Vulnerability. Journal of Neuroscience, 30(36), 12138-12150. https://doi.org/10.1523/JNEUROSCI.3245-10.2010

Benekareddy, M., Vadodaria, K. C., Nair, A. R., \& Vaidya, V. A. (2011). Postnatal Serotonin Type 2 Receptor Blockade Prevents the Emergence of Anxiety Behavior, Dysregulated Stress-Induced Immediate Early Gene Responses, and Specific Transcriptional Changes that Arise Following Early Life Stress. Biological Psychiatry, 70(11), 1024-1032. https://doi.org/10.1016/j.biopsych.2011.08.005

Blatt, G. J., Jin-Chung, C., Rosene, D. L., Volicer, L., \& Galler, J. R. (1994). Prenatal protein malnutrition effects on the serotonergic system in the hippocampal formation: An immunocytochemical, ligand binding, and neurochemical study. Brain Research Bulletin, 34(5), 507-518. https://doi.org/10.1016/0361-9230(94)90025-6 
Blier, P., \& Ward, N. M. (2003). Is there a role for 5-HT1A agonists in the treatment of depression? Biological Psychiatry, 53(3), 193-203. https://doi.org/10.1016/S00063223(02)01643-8

Bockaert, J., Claeysen, S., Bécamel, C., Dumuis, A., \& Marin, P. (2006). Neuronal 5-HT metabotropic receptors: Fine-tuning of their structure, signaling, and roles in synaptic modulation. Cell and Tissue Research, 326(2), 553-572. https://doi.org/10.1007/s00441-006$\underline{0286-1}$

Boku, S., Nakagawa, S., Toda, H., \& Hishimoto, A. (2018). Neural basis of major depressive disorder: Beyond monoamine hypothesis. Psychiatry and Clinical Neurosciences, 72(1), 3 12. https://doi.org/10.1111/pcn.12604

Boldrini, M., Santiago, A. N., Hen, R., Dwork, A. J., Rosoklija, G. B., Tamir, H., Arango, V., \& John Mann, J. (2013). Hippocampal Granule Neuron Number and Dentate Gyrus Volume in Antidepressant-Treated and Untreated Major Depression. Neuropsychopharmacology, 38(6), 1068-1077. https://doi.org/10.1038/npp.2013.5

Boldrini, M., Underwood, M. D., Mann, J. J., \& Arango, V. (2008). Serotonin-1A autoreceptor binding in the dorsal raphe nucleus of depressed suicides. Journal of Psychiatric Research, 42(6), 433-442. https://doi.org/10.1016/j.jpsychires.2007.05.004

Bonnin, A., Torii, M., Wang, L., Rakic, P., \& Levitt, P. (2007). Serotonin modulates the response of embryonic thalamocortical axons to netrin-1. Nature Neuroscience, 10(5), 588-597. https://doi.org/10.1038/nn1896

Border, R., Johnson, E. C., Evans, L. M., Smolen, A., Berley, N., Sullivan, P. F., \& Keller, M. C. (2019). No Support for Historical Candidate Gene or Candidate Gene-by-Interaction Hypotheses for Major Depression Across Multiple Large Samples. American Journal of Psychiatry, 176(5), 376-387. https://doi.org/10.1176/appi.ajp.2018.18070881

Borowsky, B., \& Hoffman, B. J. (1995). Neurotransmitter Transporters: Molecular Biology, Function, and Regulation. In R. J. Bradley \& R. A. Harris (Eds.), International Review of Neurobiology (Vol. 38, pp. 139-199). Academic Press. https://doi.org/10.1016/S00747742(08)60526-7

Bostwick, J. M., \& Pankratz, V. S. (2000). Affective Disorders and Suicide Risk: A Reexamination. American Journal of Psychiatry, 157(12), 1925-1932.

https://doi.org/10.1176/appi.ajp.157.12.1925 
Bradley, S. L., Dodelzon, K., Sandhu, H. K., \& Philibert, R. A. (2005). Relationship of serotonin transporter gene polymorphisms and haplotypes to mRNA transcription. American Journal of Medical Genetics Part B: Neuropsychiatric Genetics, 136B(1), 58-61.

https://doi.org/10.1002/ajmg.b.30185

Brain Development Maps. (2020). Postnatal Neurogenesis in the Developing Hippocampus. BrainDevelopmentMaps.Org. https://braindevelopmentmaps.org/

Bratthauer, G. L. (2010). The Avidin-Biotin Complex (ABC) Method and Other Avidin-Biotin Binding Methods. In C. Oliver \& M. C. Jamur (Eds.), Immunocytochemical Methods and Protocols (pp. 257-270). Humana Press. https://doi.org/10.1007/978-1-59745-324-0_26

Bravo, J. A., Dinan, T. G., \& Cryan, J. F. (2014). Early-life stress induces persistent alterations in 5-HT1A receptor and serotonin transporter mRNA expression in the adult rat brain. Frontiers in Molecular Neuroscience, 7. https://doi.org/10.3389/fnmol.2014.00024

Bremner, J. D., Narayan, M., Anderson, E. R., Staib, L. H., Miller, H. L., \& Charney, D. S. (2000). Hippocampal Volume Reduction in Major Depression. American Journal of Psychiatry, 157(1), 115-118. https://doi.org/10.1176/ajp.157.1.115

Bremner, J. D., Vythilingam, M., Vermetten, E., Vaccarino, V., \& Charney, D. S. (2004). Deficits in Hippocampal and Anterior Cingulate Functioning During Verbal Declarative Memory Encoding in Midlife Major Depression. American Journal of Psychiatry, 161(4), 637-645. https://doi.org/10.1176/appi.ajp.161.4.637

Brezun, J. M, \& Daszuta, A. (1999). Depletion in serotonin decreases neurogenesis in the dentate gyrus and the subventricular zone of adult rats. Neuroscience, 89(4), 999-1002. https://doi.org/10.1016/S0306-4522(98)00693-9

Brezun, J. M., \& Daszuta, A. (2000a). Serotonergic reinnervation reverses lesion-induced decreases in PSA-nCAM labeling and proliferation of hippocampal cells in adult rats. Hippocampus, 10(1), 37-46. https://doi.org/10.1002/(SICI)1098-1063(2000)10:1<37::AID$\underline{\mathrm{HIPO}} 4>3.0 . \mathrm{CO} ; 2-\mathrm{C}$

Brezun, J. M., \& Daszuta, A. (2000b). Serotonin may stimulate granule cell proliferation in the adult hippocampus, as observed in rats grafted with foetal raphe neurons. European Journal of Neuroscience, 12(1), 391-396. https://doi.org/10.1046/j.1460-9568.2000.00932.x 
Briley, M., \& Moret, C. (2010). Improvement of social adaptation in depression with serotonin and norepinephrine reuptake inhibitors. Neuropsychiatric Disease and Treatment, 6, 647655. https://doi.org/10.2147/NDT.S13171

Broadhead, W. E., Blazer, D. G., George, L. K., \& Tse, C. K. (1990). Depression, Disability Days, and Days Lost From Work in a Prospective Epidemiologic Survey. JAMA, 264(19), 25242528. https://doi.org/10.1001/jama.1990.03450190056028

Brodaty, H., Luscombe, G., Peisah, C., Anstey, K., \& Andrews, G. (2001). A 25-year longitudinal, comparison study of the outcome of depression. Psychological Medicine, 31(8), 1347-1359. https://doi.org/10.1017/S0033291701004743

Brüning, G., Liangos, O., \& Baumgarten, H. G. (1997). Prenatal development of the serotonin transporter in mouse brain. Cell and Tissue Research, 289(2), 211-221. https://doi.org/10.1007/s004410050868

Brunoni, A. R., Lopes, M., \& Fregni, F. (2008). A systematic review and meta-analysis of clinical studies on major depression and BDNF levels: Implications for the role of neuroplasticity in depression. International Journal of Neuropsychopharmacology, 11(8), 1169-1180. https://doi.org/10.1017/S1461145708009309

Burt, D. B., Zembar, M. J., \& Niederehe, G. (1995). Depression and memory impairment: A metaanalysis of the association, its pattern, and specificity. Psychological Bulletin, 117(2), 285305. https://doi.org/10.1037/0033-2909.117.2.285

Buznikov, G. A., Lambert, W. H., \& Lauder, J. M. (2001). Serotonin and serotonin-like substances as regulators of early embryogenesis and morphogenesis. Cell and Tissue Research, 305(2), 177-186. https://doi.org/10.1007/s004410100408

Cai, X., Kallarackal, A. J., Kvarta, M. D., Goluskin, S., Gaylor, K., Bailey, A. M., Lee, H.-K., Huganir, R. L., \& Thompson, S. M. (2013). Local potentiation of excitatory synapses by serotonin and its alteration in rodent models of depression. Nature Neuroscience, 16(4), 464472. https://doi.org/10.1038/nn.3355

Caldwell, M. A., He, X., \& Svendsen, C. N. (2005). 5-Bromo-2'-deoxyuridine is selectively toxic to neuronal precursors in vitro. European Journal of Neuroscience, 22(11), 2965-2970. https://doi.org/10.1111/j.1460-9568.2005.04504.x 
Calvano, C. D., Monopoli, A., Cataldi, T. R. I., \& Palmisano, F. (2018). MALDI matrices for low molecular weight compounds: An endless story? Analytical and Bioanalytical Chemistry, 410(17), 4015-4038. https://doi.org/10.1007/s00216-018-1014-X

Cameron, H. A., \& Gould, E. (1994). Adult neurogenesis is regulated by adrenal steroids in the dentate gyrus. Neuroscience, 61(2), 203-209. https://doi.org/10.1016/0306-4522(94)90224-0

Cameron, H. A., Woolley, C. S., McEwen, B. S., \& Gould, E. (1993). Differentiation of newly born neurons and glia in the dentate gyrus of the adult rat. Neuroscience, 56(2), 337-344. https://doi.org/10.1016/0306-4522(93)90335-D

Cameron, H. A., \& Mckay, R. D. G. (2001). Adult neurogenesis produces a large pool of new granule cells in the dentate gyrus. Journal of Comparative Neurology, 435(4), 406-417. https://doi.org/10.1002/cne.1040

Campbell, S., \& MacQueen, G. (2004). The role of the hippocampus in the pathophysiology of major depression. Journal of Psychiatry \& Neuroscience, 29(6), 417-426.

Campbell, S., Marriott, M., Nahmias, C., \& MacQueen, G. M. (2004). Lower Hippocampal Volume in Patients Suffering From Depression: A Meta-Analysis. American Journal of Psychiatry, 161(4), 598-607. https://doi.org/10.1176/appi.ajp.161.4.598

Caprioli, R. M., Farmer, T. B., \& Gile, J. (1997). Molecular Imaging of Biological Samples: Localization of Peptides and Proteins Using MALDI-TOF MS. Analytical Chemistry, 69(23), 4751-4760. https://doi.org/10.1021/ac970888i

Carhart-Harris, R., \& Nutt, D. (2017). Serotonin and brain function: A tale of two receptors. Journal of Psychopharmacology, 31(9), 1091-1120.

https://doi.org/10.1177/0269881117725915

Cases, O., Lebrand, C., Giros, B., Vitalis, T., Maeyer, E. D., Caron, M. G., Price, D. J., Gaspar, P., \& Seif, I. (1998). Plasma Membrane Transporters of Serotonin, Dopamine, and Norepinephrine Mediate Serotonin Accumulation in Atypical Locations in the Developing Brain of Monoamine Oxidase A Knock-Outs. Journal of Neuroscience, 18(17), 6914-6927. https://doi.org/10.1523/JNEUROSCI.18-17-06914.1998

Celada, P., Bortolozzi, A., \& Artigas, F. (2013). Serotonin 5-HT1A Receptors as Targets for Agents to Treat Psychiatric Disorders: Rationale and Current Status of Research. CNS Drugs, 27(9), 703-716. https://doi.org/10.1007/s40263-013-0071-0 
Cervilla, J. A., Rivera, M., Molina, E., Torres-González, F., Bellón, J. A., Moreno, B., Luna, J. de D., Lorente, J. A., Diego-Otero, Y. de, King, M., Nazareth, I., \& Gutiérrez, B. (2006). The 5HTTLPR s/s genotype at the serotonin transporter gene (SLC6A4) increases the risk for depression in a large cohort of primary care attendees: The PREDICT-gene study. American Journal of Medical Genetics Part B: Neuropsychiatric Genetics, 141B(8), 912-917. https://doi.org/10.1002/ajmg.b.30455

Chacon, A., Zagol-Ikapitte, I., Amarnath, V., Reyzer, M. L., Oates, J. A., Caprioli, R. M., \& Boutaud, O. (2011). On-tissue chemical derivatization of 3-methoxysalicylamine for MALDI-imaging mass spectrometry. Journal of Mass Spectrometry, 46(8), 840-846. https://doi.org/10.1002/jms.1958

Chaji, D., Venkatesh, V. S., Shirao, T., Day, D. J., \& Ellenbroek, B. A. (2021). Genetic Knockout of the Serotonin Reuptake Transporter Results in the Reduction of Dendritic Spines in In vitro Rat Cortical Neuronal Culture. Journal of Molecular Neuroscience. https://doi.org/10.1007/s12031-020-01764-9

Chalmers, D. T., Kwak, S. P., Mansour, A., Akil, H., \& Watson, S. J. (1993). Corticosteroids regulate brain hippocampal 5-HT1A receptor mRNA expression. Journal of Neuroscience, 13(3), 914-923. https://doi.org/10.1523/JNEUROSCI.13-03-00914.1993

Chase, H. W., Moses-Kolko, E. L., Zevallos, C., Wisner, K. L., \& Phillips, M. L. (2014). Disrupted posterior cingulate-amygdala connectivity in postpartum depressed women as measured with resting BOLD fMRI. Social Cognitive and Affective Neuroscience, 9(8), 1069-1075. https://doi.org/10.1093/scan/nst083

Chaurand, P., Cornett, D. S., \& Caprioli, R. M. (2006). Molecular imaging of thin mammalian tissue sections by mass spectrometry. Current Opinion in Biotechnology, 17(4), 431-436. https://doi.org/10.1016/j.copbio.2006.06.002

Chaurand, P., Norris, J. L., Cornett, D. S., Mobley, J. A., \& Caprioli, R. M. (2006z). New Developments in Profiling and Imaging of Proteins from Tissue Sections by MALDI Mass Spectrometry. Journal of Proteome Research, 5(11), 2889-2900. https://doi.org/10.1021/pr060346u

Chaurand, P., Schwartz, S. A., \& Caprioli, R. M. (2002). Imaging mass spectrometry: A new tool to investigate the spatial organization of peptides and proteins in mammalian tissue sections. 
Current Opinion in Chemical Biology, 6(5), 676-681. https://doi.org/10.1016/S1367$\underline{5931(02) 00370-8}$

Chen, F., Madsen, T. M., Wegener, G., \& Nyengaard, J. R. (2010). Imipramine treatment increases the number of hippocampal synapses and neurons in a genetic animal model of depression. Hippocampus, 20(12), 1376-1384. https://doi.org/10.1002/hipo.20718

Choi, B. H. (1988). Developmental events during the early stages of cerebral cortical neurogenesis in man. Acta Neuropathologica, 75(5), 441-447. https://doi.org/10.1007/BF00687130

Chugani, D. C. (2002). Role of altered brain serotonin mechanisms in autism. Molecular Psychiatry, 7(2), S16-S17. https://doi.org/10.1038/sj.mp.4001167

Clelland, C. D., Choi, M., Romberg, C., Clemenson, G. D., Fragniere, A., Tyers, P., Jessberger, S., Saksida, L. M., Barker, R. A., Gage, F. H., \& Bussey, T. J. (2009). A Functional Role for Adult Hippocampal Neurogenesis in Spatial Pattern Separation. Science, 325(5937), 210213. https://doi.org/10.1126/science. 1173215

Cooney, G. M., Dwan, K., Greig, C. A., Lawlor, D. A., Rimer, J., Waugh, F. R., McMurdo, M., \& Mead, G. E. (2013). Exercise for depression. Cochrane Database of Systematic Reviews, 9. https://doi.org/10.1002/14651858.CD004366.pub6

Coppen, A. (1967). The biochemistry of affective disorders. The British Journal of Psychiatry: The Journal of Mental Science, 113(504), 1237-1264. https://doi.org/10.1192/bjp.113.504.1237

Cornett, D. S., Reyzer, M. L., Chaurand, P., \& Caprioli, R. M. (2007). MALDI imaging mass spectrometry: Molecular snapshots of biochemical systems. Nature Methods, 4(10), 828-833. https://doi.org/10.1038/nmeth1094

Côté, F., Fligny, C., Bayard, E., Launay, J.-M., Gershon, M. D., Mallet, J., \& Vodjdani, G. (2007). Maternal serotonin is crucial for murine embryonic development. Proceedings of the National Academy of Sciences, 104(1), 329-334. https://doi.org/10.1073/pnas.0606722104

Creative Bioarray. (n.d.). BrdU Cell Proliferation Assay. Creative Bioarray. Retrieved September 30, 2020, from https://www.creative-bioarray.com/brdu-cell-proliferation-assay.htm Cremers, T. I. F. H., Giorgetti, M., Bosker, F. J., Hogg, S., Arnt, J., Mørk, A., Honig, G., Bøgesø, K.-P., Westerink, B. H. C., den Boer, H., Wikstrom, H. V., \& Tecott, L. H. (2004). Inactivation of 5-HT 2C Receptors Potentiates Consequences of Serotonin Reuptake 
Blockade. Neuropsychopharmacology, 29(10), 1782-1789.

https://doi.org/10.1038/sj.npp.1300474

Cryan, J. F., Valentino, R. J., \& Lucki, I. (2005). Assessing substrates underlying the behavioral effects of antidepressants using the modified rat forced swimming test. Neuroscience \& Biobehavioral Reviews, 29(4), 547-569. https://doi.org/10.1016/j.neubiorev.2005.03.008

Cullen, K. R., Gee, D. G., Klimes-Dougan, B., Gabbay, V., Hulvershorn, L., Mueller, B. A., Camchong, J., Bell, C. J., Houri, A., Kumra, S., Lim, K. O., Castellanos, F. X., \& Milham, M. P. (2009). A preliminary study of functional connectivity in comorbid adolescent depression. Neuroscience Letters, 460(3), 227-231.

https://doi.org/10.1016/j.neulet.2009.05.022

Czéh, B., Michaelis, T., Watanabe, T., Frahm, J., de Biurrun, G., van Kampen, M., Bartolomucci, A., \& Fuchs, E. (2001). Stress-induced changes in cerebral metabolites, hippocampal volume, and cell proliferation are prevented by antidepressant treatment with tianeptine. Proceedings of the National Academy of Sciences, 98(22), 12796-12801.

https://doi.org/10.1073/pnas.211427898

D’Amato, R. J., Blue, M. E., Largent, B. L., Lynch, D. R., Ledbetter, D. J., Molliver, M. E., \& Snyder, S. H. (1987). Ontogeny of the serotonergic projection to rat neocortex: transient expression of a dense innervation to primary sensory areas. Proceedings of the National Academy of Sciences, 84(12), 4322-4326. https://doi.org/10.1073/pnas.84.12.4322

Daubert, E. A., \& Condron, B. G. (2010). Serotonin: A regulator of neuronal morphology and circuitry. Trends in Neurosciences, 33(9), 424-434.

https://doi.org/10.1016/j.tins.2010.05.005

De Vitry, F., Hamon, M., Catelon, J., Dubois, M., \& Thibault, J. (1986). Serotonin initiates and autoamplifies its own synthesis during mouse central nervous system development. Proceedings of the National Academy of Sciences, 83(22), 8629-8633.

https://doi.org/10.1073/pnas.83.22.8629

Debassio, W. A., Kemper, T. L., Tonkiss, J., \& Galler, J. R. (1996). Effect of Prenatal Protein Deprivation on Postnatal Granule Cell Generation in the Hippocampal Dentate Gyrus. Brain Research Bulletin, 41(6), 379-383. https://doi.org/10.1016/S0361-9230(96)00214-6

Deckersbach, T., Dougherty, D. D., Savage, C., McMurrich, S., Fischman, A. J., Nierenberg, A., Sachs, G., \& Rauch, S. L. (2006). Impaired Recruitment of the Dorsolateral Prefrontal Cortex 
and Hippocampus During Encoding in Bipolar Disorder. Biological Psychiatry, 59(2), 138146. https://doi.org/10.1016/j.biopsych.2005.06.030

Deisseroth, K., Singla, S., Toda, H., Monje, M., Palmer, T. D., \& Malenka, R. C. (2004).

Excitation-Neurogenesis Coupling in Adult Neural Stem/Progenitor Cells. Neuron, 42(4), 535-552. https://doi.org/10.1016/S0896-6273(04)00266-1

Dekeyne, A., Mannoury la Cour, C., Gobert, A., Brocco, M., Lejeune, F., Serres, F., Sharp, T., Daszuta, A., Soumier, A., Papp, M., Rivet, J.-M., Flik, G., Cremers, T. I., Muller, O., Lavielle, G., \& Millan, M. J. (2008). S32006, a novel 5-HT2C receptor antagonist displaying broad-based antidepressant and anxiolytic properties in rodent models. Psychopharmacology, 199(4), 549-568. https://doi.org/10.1007/s00213-008-1177-9

Delacre, M., Leys, C., Mora, Y. L., \& Lakens, D. (2019). Taking Parametric Assumptions Seriously: Arguments for the Use of Welch's $F$-test instead of the Classical $F$-test in OneWay ANOVA. International Review of Social Psychology, 32(1), 13. https://doi.org/10.5334/irsp.198

Demyttenaere, K., Bruffaerts, R., Posada-Villa, J., Gasquet, I., Kovess, V., Jp, L., Mc, A., S, B., G, de G., P, M., G, P., T, K., N, K., Y, O., T, T., H, U., Eg, K., Ja, F., An, K., ... undefined. (2004). Prevalence, severity, and unmet need for treatment of mental disorders in the World Health Organization World Mental Health Surveys. JAMA, 291(21), 2581-2590. https://doi.org/10.1001/jama.291.21.2581

Descarries, L., \& Riad, M. (2012). Effects of the antidepressant fluoxetine on the subcellular localization of 5-HT1A receptors and SERT. Philosophical Transactions of the Royal Society B: Biological Sciences, 367(1601), 2416-2425. https://doi.org/10.1098/rstb.2011.0361

D'haenen, H. (2001). Imaging the serotonergic system in depression. European Archives of Psychiatry and Clinical Neuroscience, 251(2), 76-80. https://doi.org/10.1007/BF03035133

Djordjevic, A., Djordjevic, J., Elaković, I., Adzic, M., Matić, G., \& Radojcic, M. B. (2012). Fluoxetine affects hippocampal plasticity, apoptosis and depressive-like behavior of chronically isolated rats. Progress in Neuro-Psychopharmacology and Biological Psychiatry, 36(1), 92-100. https://doi.org/10.1016/j.pnpbp.2011.10.006 
Dranovsky, A., \& Hen, R. (2006). Hippocampal Neurogenesis: Regulation by Stress and Antidepressants. Biological Psychiatry, 59(12), 1136-1143.

https://doi.org/10.1016/j.biopsych.2006.03.082

Drapeau, E., Mayo, W., Aurousseau, C., Moal, M. L., Piazza, P.-V., \& Abrous, D. N. (2003).

Spatial memory performances of aged rats in the water maze predict levels of hippocampal neurogenesis. Proceedings of the National Academy of Sciences, 100(24), 14385-14390. https://doi.org/10.1073/pnas.2334169100

Dreisewerd, K. (2003). The Desorption Process in MALDI. Chemical Reviews, 103(2), 395-426. https://doi.org/10.1021/cr010375i

Dreisewerd, K., Schürenberg, M., Karas, M., \& Hillenkamp, F. (1995). Influence of the laser intensity and spot size on the desorption of molecules and ions in matrix-assisted laser desorption/ionization with a uniform beam profile. International Journal of Mass Spectrometry and Ion Processes, 141(2), 127-148. https://doi.org/10.1016/0168$\underline{1176(94) 04108-\mathrm{J}}$

Drury, S. S., Gleason, M. M., Theall, K. P., Smyke, A. T., Nelson, C. A., Fox, N. A., \& Zeanah, C. H. (2012). Genetic sensitivity to the caregiving context: The influence of 5httlpr and BDNF val66met on indiscriminate social behavior. Physiology \& Behavior, 106(5), 728-735. https://doi.org/10.1016/j.physbeh.2011.11.014

Druss, B. G., Hwang, I., Petukhova, M., Sampson, N. A., Wang, P. S., \& Kessler, R. C. (2009). Impairment in role functioning in mental and chronic medical disorders in the United States: Results from the National Comorbidity Survey Replication. Molecular Psychiatry, 14(7), 728-737. https://doi.org/10.1038/mp.2008.13

Duan, X., Kang, E., Liu, C. Y., Ming, G., \& Song, H. (2008). Development of neural stem cell in the adult brain. Current Opinion in Neurobiology, 18(1), 108-115. https://doi.org/10.1016/j.conb.2008.04.001

Dueñas, M. E., Larson, E. A., \& Lee, Y. J. (2019). Toward Mass Spectrometry Imaging in the Metabolomics Scale: Increasing Metabolic Coverage Through Multiple On-Tissue Chemical Modifications. Frontiers in Plant Science, 10. https://doi.org/10.3389/fpls.2019.00860

Duman, R. S. (2004). Depression: A case of neuronal life and death? Biological Psychiatry, 56(3), 140-145. https://doi.org/10.1016/j.biopsych.2004.02.033 
Duman, R. S., \& Monteggia, L. M. (2006). A Neurotrophic Model for Stress-Related Mood Disorders. Biological Psychiatry, 59(12), 1116-1127. https://doi.org/10.1016/j.biopsych.2006.02.013

Ehninger, D., \& Kempermann, G. (2008). Neurogenesis in the adult hippocampus. Cell and Tissue Research, 331(1), 243-250. https://doi.org/10.1007/s00441-007-0478-3

Eichenbaum, H. (2010). Hippocampus. In The Corsini Encyclopedia of Psychology (pp. 1-2). American Cancer Society. https://doi.org/10.1002/9780470479216.corpsy0412

Eisch, A., Cameron, H., Encinas, J., Meltzer, L., Ming, G.-L., \& Wadiche, L. (2008). Adult Neurogenesis, Mental Health, and Mental Illness: Hope or Hype? The Journal of Neuroscience : The Official Journal of the Society for Neuroscience, 28, 11785-11791. https://doi.org/10.1523/JNEUROSCI.3798-08.2008

Ellis, P. M., \& Salmond, C. (1994). Is platelet imipramine binding reduced in depression? A metaanalysis. Biological Psychiatry, 36(5), 292-299. https://doi.org/10.1016/0006$\underline{3223(94) 90626-2}$

Encinas, J. M., Vaahtokari, A., \& Enikolopov, G. (2006). Fluoxetine targets early progenitor cells in the adult brain. Proceedings of the National Academy of Sciences, 103(21), 8233-8238. https://doi.org/10.1073/pnas.0601992103

Eriksson, P. S., Perfilieva, E., Björk-Eriksson, T., Alborn, A.-M., Nordborg, C., Peterson, D. A., \& Gage, F. H. (1998). Neurogenesis in the adult human hippocampus. Nature Medicine, 4(11), 1313-1317. https://doi.org/10.1038/3305

Esteve, C., Tolner, E. A., Shyti, R., van den Maagdenberg, A. M. J. M., \& McDonnell, L. A. (2016). Mass spectrometry imaging of amino neurotransmitters: A comparison of derivatization methods and application in mouse brain tissue. Metabolomics, 12(2), 30. https://doi.org/10.1007/s11306-015-0926-0

Faber, K. M., \& Haring, J. H. (1999). Synaptogenesis in the postnatal rat fascia dentata is influenced by 5-HT1a receptor activation. Developmental Brain Research, 114(2), 245-252. https://doi.org/10.1016/S0165-3806(99)00036-X

Fabre, V., Beaufour, C., Evrard, A., Rioux, A., Hanoun, N., Lesch, K. P., Murphy, D. L., Lanfumey, L., Hamon, M., \& Martres, M.-P. (2000). Altered expression and functions of serotonin 5-HT1A and 5-HT1B receptors in knock-out mice lacking the 5-HT transporter. 
European Journal of Neuroscience, 12(7), 2299-2310. https://doi.org/10.1046/j.14609568.2000.00126.x

Fanselow, M. S., \& Dong, H.-W. (2010). Are the Dorsal and Ventral Hippocampus Functionally Distinct Structures? Neuron, 65(1), 7-19. https://doi.org/10.1016/j.neuron.2009.11.031

Feldman, D. E. (2009). Synaptic Mechanisms for Plasticity in Neocortex. Annual Review of Neuroscience, 32(1), 33-55. https://doi.org/10.1146/annurev.neuro.051508.135516

Feldman, R., \& Bakermans-Kranenburg, M. J. (2017). Oxytocin: A parenting hormone. Current Opinion in Psychology, 15, 13-18. https://doi.org/10.1016/j.copsyc.2017.02.011

Ferrés-Coy, A., Pilar-Cuellar, F., Vidal, R., Paz, V., Masana, M., Cortés, R., Carmona, M. C., Campa, L., Pazos, Á., Montefeltro, A., Valdizán, E. M., Artigas, F., \& Bortolozzi, A. (2013). RNAi-mediated serotonin transporter suppression rapidly increases serotonergic neurotransmission and hippocampal neurogenesis. Translational Psychiatry, 3(1), e211e211. https://doi.org/10.1038/tp.2012.135

Figueiredo, H. F., Bodie, B. L., Tauchi, M., Dolgas, C. M., \& Herman, J. P. (2003). Stress Integration after Acute and Chronic Predator Stress: Differential Activation of Central Stress Circuitry and Sensitization of the Hypothalamo-Pituitary-Adrenocortical Axis. Endocrinology, 144(12), 5249-5258. https://doi.org/10.1210/en.2003-0713

Flugge, G. (1995). Dynamics of central nervous 5-HT1A-receptors under psychosocial stress. Journal of Neuroscience, 15(11), 7132-7140. https://doi.org/10.1523/JNEUROSCI.15-11$\underline{07132.1995}$

Freund, T. F., Gulyás, A. I., Acsády, L., Görcs, T., \& Tóth, K. (1990). Serotonergic control of the hippocampus via local inhibitory interneurons. Proceedings of the National Academy of Sciences of the United States of America, 87(21), 8501-8505.

Frodl, T., Reinhold, E., Koutsouleris, N., Reiser, M., \& Meisenzahl, E. M. (2010). Interaction of childhood stress with hippocampus and prefrontal cortex volume reduction in major depression. Journal of Psychiatric Research, 44(13), 799-807. https://doi.org/10.1016/j.jpsychires.2010.01.006

Fuchs, B. (2014). Mass spectrometry and inflammation-MS methods to study oxidation and enzyme-induced changes of phospholipids. Analytical and Bioanalytical Chemistry; Heidelberg, 406(5), 1291-1306. http://dx.doi.org/10.1007/s00216-013-7534-5 
Furlong, R. A., Ho, L., Walsh, C., Rubinsztein, J. S., Jain, S., Paykel, E. S., Easton, D. F., \& Rubinsztein, D. C. (1998). Analysis and meta-analysis of two serotonin transporter gene polymorphisms in bipolar and unipolar affective disorders. American Journal of Medical Genetics, 81(1), 58-63. https://doi.org/10.1002/(SICI)1096-8628(19980207)81:1<58::AID$\underline{\mathrm{AJMG} 11>3.0 . \mathrm{CO} ; 2-\mathrm{V}}$

Gage, F. H. (2002). Neurogenesis in the Adult Brain. Journal of Neuroscience, 22(3), 612-613. https://doi.org/10.1523/JNEUROSCI.22-03-00612.2002

Garcia-Garcia, A. L., Newman-Tancredi, A., \& Leonardo, E. D. (2014). P5-HT1A receptors in mood and anxiety: Recent insights into autoreceptor versus heteroreceptor function. Psychopharmacology, 231(4), 623-636. https://doi.org/10.1007/s00213-013-3389-x

Gaspar, P., Cases, O., \& Maroteaux, L. (2003). The developmental role of serotonin: news from mouse molecular genetics. Nature Reviews Neuroscience, 4(12), 1002-1012. https://doi.org/10.1038/nrn1256

Ge, S., Yang, C., Hsu, K., Ming, G., \& Song, H. (2007). A Critical Period for Enhanced Synaptic Plasticity in Newly Generated Neurons of the Adult Brain. Neuron, 54(4), 559-566. https://doi.org/10.1016/j.neuron.2007.05.002

Gérard, C., Langlois, X., Gingrich, J., Doucet, E., Vergé, D., Kia, H. K., Raisman, R., Gozlan, H., El Mestikawy, S., \& Hamon, M. (1994). Production and characterization of polyclonal antibodies recognizing the intracytoplasmic third loop of the 5-hydroxytryptamine1A receptor. Neuroscience, 62(3), 721-739. https://doi.org/10.1016/0306-4522(94)90472-3

Giap, B. T., Jong, C. N., Ricker, J. H., Cullen, N. K., \& Zafonte, R. D. (2000). The Hippocampus: Anatomy, Pathophysiology, and Regenerative Capacity. The Journal of Head Trauma Rehabilitation, 15(3), 875-894.

Gibbons, R. D., \& Davis, J. M. (1986). Consistent evidence for a biological subtype of depression characterized by low CSF monoamine levels. Acta Psychiatrica Scandinavica, 74(1), 8-12. https://doi.org/10.1111/j.1600-0447.1986.tb06219.x

Gilmer, W. S., Trivedi, M. H., Rush, A. J., Wisniewski, S. R., Luther, J., Howland, R. H., Yohanna, D., Khan, A., \& Alpert, J. (2005). Factors associated with chronic depressive episodes: A preliminary report from the STAR-D project. Acta Psychiatrica Scandinavica, 112(6), 425-433. https://doi.org/10.1111/j.1600-0447.2005.00633.x 
Gingrich, J. A., \& Hen, R. (2001). Dissecting the role of the serotonin system in neuropsychiatric disorders using knockout mice. Psychopharmacology, 155(1), 1-10.

https://doi.org/10.1007/s002130000573

Gold, P. W., \& Chrousos, G. P. (2002). Organization of the stress system and its dysregulation in melancholic and atypical depression: High vs low CRH/NE states. Molecular Psychiatry, 7(3), 254-275. https://doi.org/10.1038/sj.mp.4001032

Gómez, R. L., \& Edgin, J. O. (2016). The extended trajectory of hippocampal development: Implications for early memory development and disorder. Developmental Cognitive Neuroscience, 18, 57-69. https://doi.org/10.1016/j.den.2015.08.009

Gould, E., Beylin, A., Tanapat, P., Reeves, A., \& Shors, T. J. (1999). Learning enhances adult neurogenesis in the hippocampal formation. Nature Neuroscience, 2(3), 260-265. https://doi.org/10.1038/6365

Gould, E., Cameron, H. A., Daniels, D. C., Woolley, C. S., \& McEwen, B. S. (1992). Adrenal hormones suppress cell division in the adult rat dentate gyrus. Journal of Neuroscience, 12(9), 3642-3650. https://doi.org/10.1523/JNEUROSCI.12-09-03642.1992

Gould, E. (1999). Serotonin and Hippocampal Neurogenesis. Neuropsychopharmacology, 21(1), 46-51. https://doi.org/10.1016/S0893-133X(99)00045-7

Gould, E., Tanapat, P., Hastings, N. B., \& Shors, T. J. (1999). Neurogenesis in adulthood: A possible role in learning. Trends in Cognitive Sciences, 3(5), 186-192. https://doi.org/10.1016/S1364-6613(99)01310-8

Gould, E., Tanapat, P., McEwen, B. S., Flügge, G., \& Fuchs, E. (1998). Proliferation of granule cell precursors in the dentate gyrus of adult monkeys is diminished by stress. Proceedings of the National Academy of Sciences, 95(6), 3168-3171. https://doi.org/10.1073/pnas.95.6.3168

Gourevitch, R., Rocher, C., Pen, G. L., Krebs, M.-O., \& Jay, T. M. (2004). Working memory deficits in adult rats after prenatal disruption of neurogenesis. Behavioural Pharmacology, 15(4), 287-292. https://doi.org/10.1097/01.fbp.0000135703.48799.71

Gratzner, H. G. (1982). Monoclonal antibody to 5-bromo- and 5-iododeoxyuridine: A new reagent for detection of DNA replication. Science (New York, N.Y.), 218(4571), 474-475. https://doi.org/10.1126/science.7123245 
Gray, N. A., Milak, M. S., DeLorenzo, C., Ogden, R. T., Huang, Y., Mann, J. J., \& Parsey, R. V. (2013). Antidepressant Treatment Reduces Serotonin-1A Autoreceptor Binding in Major Depressive Disorder. Biological Psychiatry, 74(1), 26-31.

https://doi.org/10.1016/j.biopsych.2012.11.012

Greicius, M. D., Flores, B. H., Menon, V., Glover, G. H., Solvason, H. B., Kenna, H., Reiss, A. L., \& Schatzberg, A. F. (2007). Resting-State Functional Connectivity in Major Depression: Abnormally Increased Contributions from Subgenual Cingulate Cortex and Thalamus. Biological Psychiatry, 62(5), 429-437. https://doi.org/10.1016/j.biopsych.2006.09.020

Gross, C., Zhuang, X., Stark, K., Ramboz, S., Oosting, R., Kirby, L., Santarelli, L., Beck, S., \& Hen, R. (2002). Serotonin 1A receptor acts during development to establish normal anxietylike behaviour in the adult. Nature, 416(6879), 396-400. https://doi.org/10.1038/416396a

Guimarães, F. S., Del Bel, E. A., Padovan, C. M., Netto, S. M., \& de Almeida, R. T. (1993). Hippocampal 5-HT receptors and consolidation of stressful memories. Behavioural Brain Research, 58(1), 133-139. https://doi.org/10.1016/0166-4328(93)90098-B

Guo, Z., \& He, L. (2007). A binary matrix for background suppression in MALDI-MS of small molecules. Analytical and Bioanalytical Chemistry, 387(5), 1939-1944. https://doi.org/10.1007/s00216-006-1100-3

Gutiérrez, B., Pintor, L., Gastó, C., Rosa, A., Bertranpetit, J., Vieta, E., \& Fañanás, L. (1998). Variability in the serotonin transporter gene and increased risk for major depression with melancholia. Human Genetics, 103(3), 319-322. https://doi.org/10.1007/s004390050823

Haddjeri, N., Blier, P., \& Montigny, C. de. (1998). Long-Term Antidepressant Treatments Result in a Tonic Activation of Forebrain 5-HT1A Receptors. Journal of Neuroscience, 18(23), 10150-10156. https://doi.org/10.1523/JNEUROSCI.18-23-10150.1998

Hamet, P., \& Tremblay, J. (2005). Genetics and genomics of depression. Metabolism, 54(5, Supplement), 10-15. https://doi.org/10.1016/j.metabol.2005.01.006

Hamon, M., \& Blier, P. (2013). Monoamine neurocircuitry in depression and strategies for new treatments. Progress in Neuro-Psychopharmacology and Biological Psychiatry, 45, 54-63. https://doi.org/10.1016/j.pnpbp.2013.04.009

Hancock, A., Priester, C., Kidder, E., \& Keith, J. R. (2009). Does 5-bromo-2'-deoxyuridine (BrdU) disrupt cell proliferation and neuronal maturation in the adult rat hippocampus in 
vivo? Behavioural Brain Research, 199(2), 218-221.

https://doi.org/10.1016/j.bbr.2008.11.050

Hanrieder, J., Phan, N. T. N., Kurczy, M. E., \& Ewing, A. G. (2013). Imaging Mass Spectrometry in Neuroscience. ACS Chemical Neuroscience, 4(5), 666-679. https://doi.org/10.1021/cn400053c

Hansson, S. R., Mezey, É., \& Hoffman, B. J. (1999). Serotonin transporter messenger RNA expression in neural crest-derived structures and sensory pathways of the developing rat embryo. Neuroscience, 89(1), 243-265. https://doi.org/10.1016/S0306-4522(98)00281-4

Haring, J. H., Hagan, A., Olson, J., \& Rodgers, B. (1993). Hippocampal serotonin levels influence the expression of S100ß detected by immunocytochemistry. Brain Research, 631(1), 119123. https://doi.org/10.1016/0006-8993(93)91195-X

Hariri, A. R., Mattay, V. S., Tessitore, A., Kolachana, B., Fera, F., Goldman, D., Egan, M. F., \& Weinberger, D. R. (2002). Serotonin Transporter Genetic Variation and the Response of the Human Amygdala. Science, 297(5580), 400-403. https://doi.org/10.1126/science.1071829

Harvey, J. A. (2003). Role of the Serotonin 5-HT2A Receptor in Learning. Learning \& Memory, 10(5), 355-362. https://doi.org/10.1101/1m.60803

Hayakawa, H., Shimizu, M., Nishida, A., Motohashi, N., \& Yamawaki, S. (1994). Increase in Serotonin 1A Receptors in the Dentate Gyrus as Revealed by Autoradiographic Analysis Following Repeated Electroconvulsive Shock But Not Imipramine Treatment. Neuropsychobiology, 30(2-3), 53-56. https://doi.org/10.1159/000119413

Haydon, P. G., McCobb, D. P., \& Kater, S. B. (1984). Serotonin selectively inhibits growth cone motility and synaptogenesis of specific identified neurons. Science, 226(4674), 561-564. https://doi.org/10.1126/science.6093252

Heils, A., Teufel, A., Petri, S., Stöber, G., Riederer, P., Bengel, D., \& Lesch, K. P. (1996). Allelic Variation of Human Serotonin Transporter Gene Expression. Journal of Neurochemistry, 66(6), 2621-2624. https://doi.org/10.1046/j.1471-4159.1996.66062621.X

Heiming, R. S., Jansen, F., Lewejohann, L., Kaiser, S., Schmitt, A., Lesch, K. P., \& Sachser, N. (2009). Living in a dangerous world: The shaping of behavioral profile by early environment and 5-HTT genotype. Frontiers in Behavioral Neuroscience, 3. https://doi.org/10.3389/neuro.08.026.2009 
Heiming, R. S., Mönning, A., Jansen, F., Kloke, V., Lesch, K.-P., \& Sachser, N. (2013). To attack, or not to attack? The role of serotonin transporter genotype in the display of maternal aggression. Behavioural Brain Research, 242, 135-141.

https://doi.org/10.1016/j.bbr.2012.12.045

Heinz, A., Braus, D. F., Smolka, M. N., Wrase, J., Puls, I., Hermann, D., Klein, S., Grüsser, S. M., Flor, H., Schumann, G., Mann, K., \& Büchel, C. (2005). Amygdala-prefrontal coupling depends on a genetic variation of the serotonin transporter. Nature Neuroscience, 8(1), 2021. https://doi.org/10.1038/nn1366

Herman, J. P., Adams, D., \& Prewitt, C. (1995). Regulatory Changes in Neuroendocrine StressIntegrative Circuitry Produced by a Variable Stress Paradigm. Neuroendocrinology, 61(2), 180-190. https://doi.org/10.1159/000126839

Hervás, I., Vilaró, M. T., Romero, L., Scorza, Mc., Mengod, G., \& Artigas, F. (2001).

Desensitization of 5-HT 1A Autoreceptors by a Low Chronic Fluoxetine Dose Effect of the Concurrent Administration of WAY-100635. Neuropsychopharmacology, 24(1), 11-20. https://doi.org/10.1016/S0893-133X(00)00175-5

Hillenkamp, F., Karas, M., Beavis, R. C., \& Chait, B. T. (1991). Matrix-assisted laser desorption/ionization mass spectrometry of biopolymers. Analytical Chemistry, 63(24), 1193A-1203A. https://doi.org/10.1021/ac00024a002

Hindmarch, I. (2001). Expanding the horizons of depression: Beyond the monoamine hypothesis. Human Psychopharmacology: Clinical and Experimental, 16(3), 203-218. https://doi.org/10.1002/hup.288

Holmes, A., Murphy, D. L., \& Crawley, J. N. (2003). Abnormal behavioral phenotypes of serotonin transporter knockout mice: Parallels with human anxiety and depression. Biological Psychiatry, 54(10), 953-959. https://doi.org/10.1016/j.biopsych.2003.09.003

Holschneider, D. P., Chen, K., Seif, I., \& Shih, J. C. (2001). Biochemical, behavioral, physiologic, and neurodevelopmental changes in mice deficient in monoamine oxidase A or B. Brain Research Bulletin, 56(5), 453-462. https://doi.org/10.1016/S0361-9230(01)00613-X

Holtmaat, A., \& Svoboda, K. (2009). Experience-dependent structural synaptic plasticity in the mammalian brain. Nature Reviews Neuroscience, 10(9), 647-658.

https://doi.org/10.1038/nrn2699 
Homberg, J. R., Olivier, J. D. A., Smits, B. M. G., Mul, J. D., Mudde, J., Verheul, M., Nieuwenhuizen, O. F. M., Cools, A. R., Ronken, E., Cremers, T., Schoffelmeer, A. N. M., Ellenbroek, B. A., \& Cuppen, E. (2007). Characterization of the serotonin transporter knockout rat: A selective change in the functioning of the serotonergic system. Neuroscience, 146(4), 1662-1676. https://doi.org/10.1016/j.neuroscience.2007.03.030

Homberg, Judith R., Schubert, D., \& Gaspar, P. (2010). New perspectives on the neurodevelopmental effects of SSRIs. Trends in Pharmacological Sciences, 31(2), 60-65. https://doi.org/10.1016/j.tips.2009.11.003

Homberg, Judith Regina, Molteni, R., Calabrese, F., \& Riva, M. A. (2014). The serotonin-BDNF duo: Developmental implications for the vulnerability to psychopathology. Neuroscience \& Biobehavioral Reviews, 43, 35-47. https://doi.org/10.1016/j.neubiorev.2014.03.012

Houwing, D. J., Ramsteijn, A. S., Riemersma, I. W., \& Olivier, J. D. A. (2019). Maternal separation induces anhedonia in female heterozygous serotonin transporter knockout rats. Behavioural Brain Research, 356, 204-207. https://doi.org/10.1016/j.bbr.2018.08.031

Hsu, S.-M. (1990). Immunohistochemistry. In M. Wilchek \& E. A. Bayer (Eds.), Methods in Enzymology (Vol. 184, pp. 357-363). Academic Press. https://doi.org/10.1016/0076$\underline{6879(90) 84293-\mathrm{P}}$

Hu, M., Sun, Y.-J., Zhou, Q.-G., Auberson, Y. P., Chen, L., Hu, Y., Luo, C.-X., Wu, J.-Y., Zhu, D.-Y., \& Li, L.-X. (2009). Reduced spatial learning in mice treated with NVP-AAM077 through down-regulating neurogenesis. European Journal of Pharmacology, 622(1), 37-44. https://doi.org/10.1016/j.ejphar.2009.09.031

Huang, G.-J., \& Herbert, J. (2005). The role of 5-HT1A receptors in the proliferation and survival of progenitor cells in the dentate gyrus of the adult hippocampus and their regulation by corticoids. Neuroscience, 135(3), 803-813. https://doi.org/10.1016/j.neuroscience.2005.05.056

Huang, W. Q., Zhang, C. L., Di, X. Y., \& Zhang, R. Q. (1998). Studies on the localization of 5hydroxytryptamine and its receptors in human placenta. Placenta, 19(8), 655-661. https://doi.org/10.1016/S0143-4004(98)90027-3 
Ilsley, J. E., Moffoot, A. P. R., \& O’Carroll, R. E. (1995). An analysis of memory dysfunction in major depression. Journal of Affective Disorders, 35(1), 1-9. https://doi.org/10.1016/0165$\underline{0327(95) 00032-\mathrm{I}}$

Imayoshi, I., Sakamoto, M., Ohtsuka, T., Takao, K., Miyakawa, T., Yamaguchi, M., Mori, K., Ikeda, T., Itohara, S., \& Kageyama, R. (2008). Roles of continuous neurogenesis in the structural and functional integrity of the adult forebrain. Nature Neuroscience, 11(10), 11531161. https://doi.org/10.1038/nn.2185

Imoto, Y., Kira, T., Sukeno, M., Nishitani, N., Nagayasu, K., Nakagawa, T., Kaneko, S., Kobayashi, K., \& Segi-Nishida, E. (2015). Role of the 5-HT4 receptor in chronic fluoxetine treatment-induced neurogenic activity and granule cell dematuration in the dentate gyrus. Molecular Brain, 8(1), 29. https://doi.org/10.1186/s13041-015-0120-3

Insausti, R., \& Amaral, D. G. (2003). Hippocampal Formation. The Human Nervous System: Second Edition, 871-914. https://doi.org/10.1016/B978-012547626-3/50024-7

Jacobs, B. L., van Praag, H., \& Gage, F. H. (2000). Adult brain neurogenesis and psychiatry: A novel theory of depression. Molecular Psychiatry, 5(3), 262-269.

https://doi.org/10.1038/sj.mp.4000712

Jacobson, S., \& Marcus, E. M. (Eds.). (2008). Limbic System, the Temporal Lobe, and Prefrontal Cortex. In Neuroanatomy for the Neuroscientist (pp. 337-373). Springer US.

https://doi.org/10.1007/978-0-387-70971-0_14

Jankord, R., \& Herman, J. P. (2008). Limbic regulation of hypothalamo-pituitary-adrenocortical function during acute and chronic stress. Annals of the New York Academy of Sciences, 1148, 64-73. https://doi.org/10.1196/annals.1410.012

Jarrett, M. E., Kohen, R., Cain, K. C., Burr, R. L., Poppe, A., Navaja, G. P., \& Heitkemper, M. M. (2007). Relationship of SERT Polymorphisms to Depressive and Anxiety Symptoms in Irritable Bowel Syndrome. Biological Research For Nursing, 9(2), 161-169. https://doi.org/10.1177/1099800407307822

Jayatissa, M. N., Bisgaard, C., Tingström, A., Papp, M., \& Wiborg, O. (2006). Hippocampal Cytogenesis Correlates to Escitalopram-Mediated Recovery in a Chronic Mild Stress Rat Model of Depression. Neuropsychopharmacology, 31(11), 2395-2404.

https://doi.org/10.1038/sj.npp.1301041 
Jessberger, S., \& Kempermann, G. (2003). Adult-born hippocampal neurons mature into activitydependent responsiveness. European Journal of Neuroscience, 18(10), 2707-2712. https://doi.org/10.1111/j.1460-9568.2003.02986.x

Jiang, X., Wang, J., Luo, T., \& Li, Q. (2009). Impaired hypothalamic-pituitary-adrenal axis and its feedback regulation in serotonin transporter knockout mice. Psychoneuroendocrinology, 34(3), 317-331. https://doi.org/10.1016/j.psyneuen.2008.09.011

Johns, J. M., Joyner, P. W., McMurray, M. S., Elliott, D. L., Hofler, V. E., Middleton, C. L., Knupp, K., Greenhill, K. W., Lomas, L. M., \& Walker, C. H. (2005). The effects of dopaminergic/serotonergic reuptake inhibition on maternal behavior, maternal aggression, and oxytocin in the rat. Pharmacology Biochemistry and Behavior, 81(4), 769-785. https://doi.org/10.1016/j.pbb.2005.06.001

Jørgensen, H., Riis, M., Knigge, U., Kjær, A., \& Warberg, J. (2003). Serotonin Receptors Involved in Vasopressin and Oxytocin Secretion. Journal of Neuroendocrinology, 15(3), 242-249. https://doi.org/10.1046/j.1365-2826.2003.00978.x

Joyce, P. R., Oakley-Browne, M. A., Wells, J. E., Bushnell, J. A., \& Hornblow, A. R. (1990). Birth cohort trends in major depression: Increasing rates and earlier onset in New Zealand. Journal of Affective Disorders, 18(2), 83-89. https://doi.org/10.1016/0165-0327(90)90063-E

Judd, L. L., Akiskal, H. S., Zeller, P. J., Paulus, M., Leon, A. C., Maser, J. D., Endicott, J., Coryell, W., Kunovac, J. L., Mueller, T. I., Rice, J. P., \& Keller, M. B. (2000). Psychosocial Disability During the Long-term Course of Unipolar Major Depressive Disorder. Archives of General Psychiatry, 57(4), 375-380. https://doi.org/10.1001/archpsyc.57.4.375

Kaiser, R. H., Andrews-Hanna, J. R., Wager, T. D., \& Pizzagalli, D. A. (2015). Large-Scale Network Dysfunction in Major Depressive Disorder: A Meta-analysis of Resting-State Functional Connectivity. JAMA Psychiatry, 72(6), 603-611. https://doi.org/10.1001/jamapsychiatry.2015.0071

Kalueff, A. V., Olivier, J. D. A., Nonkes, L. J. P., \& Homberg, J. R. (2010). Conserved role for the serotonin transporter gene in rat and mouse neurobehavioral endophenotypes. Neuroscience \& Biobehavioral Reviews, 34(3), 373-386. https://doi.org/10.1016/j.neubiorev.2009.08.003

Karabeg, M. M., Grauthoff, S., Kollert, S. Y., Weidner, M., Heiming, R. S., Jansen, F., Popp, S., Kaiser, S., Lesch, K.-P., Sachser, N., Schmitt, A. G., \& Lewejohann, L. (2013). 5-HTT 
Deficiency Affects Neuroplasticity and Increases Stress Sensitivity Resulting in Altered Spatial Learning Performance in the Morris Water Maze but Not in the Barnes Maze. PLOS ONE, 8(10), e78238. https://doi.org/10.1371/journal.pone.0078238

Karas, M., Bachmann, D., Bahr, U., \& Hillenkamp, F. (1987). Matrix-assisted ultraviolet laser desorption of non-volatile compounds. International Journal of Mass Spectrometry and Ion Processes, 78, 53-68. https://doi.org/10.1016/0168-1176(87)87041-6

Karas, M., Bahr, U., Ingendoh, A., \& Hillenkamp, F. (1989). Laser Desorption/Ionization Mass Spectrometry of Proteins of Mass 100000 to 250000 Dalton. Angewandte Chemie International Edition in English, 28(6), 760-761. https://doi.org/10.1002/anie.198907601

Karas, M., \& Krüger, R. (2003). Ion Formation in MALDI: The Cluster Ionization Mechanism. Chemical Reviews, 103(2), 427-440. https://doi.org/10.1021/cr010376a

Karg, K., Burmeister, M., Shedden, K., \& Sen, S. (2011). The Serotonin Transporter Promoter Variant (5-HTTLPR), Stress, and Depression Meta-analysis Revisited: Evidence of Genetic Moderation. Archives of General Psychiatry, 68(5), 444-454.

https://doi.org/10.1001/archgenpsychiatry.2010.189

Kaufman, J., Plotsky, P. M., Nemeroff, C. B., \& Charney, D. S. (2000). Effects of early adverse experiences on brain structure and function: Clinical implications. Biological Psychiatry, 48(8), 778-790. https://doi.org/10.1016/S0006-3223(00)00998-7

Kelsoe, J. R., Remick, R. A., Sadovnick, A. D., Kristbjarnarson, H., Flodman, P., Spence, M. A., Morison, M., Mroczkowski-Parker, Z., Bergesch, P., Rapaport, M. H., Mirow, A. L., Blakely, R. D., Helgason, T., \& Egeland, J. A. (1996). Genetic linkage study of bipolar disorder and the serotonin transporter. American Journal of Medical Genetics, 67(2), 215-217. https://doi.org/10.1002/(SICI)1096-8628(19960409)67:2<215::AID-AJMG14>3.0.CO;2-M

Kempermann, G., \& Gage, F. H. (2002). Genetic determinants of adult hippocampal neurogenesis correlate with acquisition, but not probe trial performance, in the water maze task. European Journal of Neuroscience, 16(1), 129-136. https://doi.org/10.1046/j.1460-9568.2002.02042.x

Kempermann, G. (2002). Why New Neurons? Possible Functions for Adult Hippocampal Neurogenesis. Journal of Neuroscience, 22(3), 635-638. https://doi.org/10.1523/JNEUROSCI.22-03-00635.2002 
Kempermann, G., Jessberger, S., Steiner, B., \& Kronenberg, G. (2004a). Milestones of neuronal development in the adult hippocampus. Trends in Neurosciences, 27(8), 447-452. https://doi.org/10.1016/j.tins.2004.05.013

Kempermann, G., Kuhn, H. G., \& Gage, F. H. (1998). Experience-Induced Neurogenesis in the Senescent Dentate Gyrus. Journal of Neuroscience, 18(9), 3206-3212. https://doi.org/10.1523/JNEUROSCI.18-09-03206.1998

Kempermann, G., Wiskott, L., \& Gage, F. H. (2004b). Functional significance of adult neurogenesis. Current Opinion in Neurobiology, 14(2), 186-191. https://doi.org/10.1016/j.conb.2004.03.001

Kennedy, N., Foy, K., Sherazi, R., McDonough, M., \& McKeon, P. (2007). Long-term social functioning after depression treated by psychiatrists: A review. Bipolar Disorders, 9(1-2), 25-37. https://doi.org/10.1111/j.1399-5618.2007.00326.x

Kesper, L.-J., \& Homberg, J. R. (2015). The neurodevelopmental effects of serotonin: A behavioural perspective. Behavioural Brain Research, 277, 3-13. https://doi.org/10.1016/j.bbr.2014.05.022

Kessler, R. C., Akiskal, H. S., Ames, M., Birnbaum, H., Greenberg, P., Hirschfeld, R., Jin, R., Merikangas, K. R., Simon, G. E., \& Wang, P. S. (2006). Prevalence and Effects of Mood Disorders on Work Performance in a Nationally Representative Sample of U.S. Workers. American Journal of Psychiatry, 163(9), 1561-1568. https://doi.org/10.1176/ajp.2006.163.9.1561

Kessler, R. C., Zhao, S., Blazer, D. G., \& Swartz, M. (1997). Prevalence, correlates, and course of minor depression and major depression in the national comorbidity survey. Journal of Affective Disorders, 45(1), 19-30. https://doi.org/10.1016/S0165-0327(97)00056-6

Khazipov, R., Zaynutdinova, D., Ogievetsky, E., Valeeva, G., Mitrukhina, O., Manent, J.-B., \& Represa, A. (2015). Atlas of the Postnatal Rat Brain in Stereotaxic Coordinates. Frontiers in Neuroanatomy, 9. https://doi.org/10.3389/fnana.2015.00161

Kikusui, T., Ichikawa, S., \& Mori, Y. (2009). Maternal deprivation by early weaning increases corticosterone and decreases hippocampal BDNF and neurogenesis in mice.

Psychoneuroendocrinology, 34(5), 762-772. https://doi.org/10.1016/j.psyneuen.2008.12.009

Kiloh, L. G., Andrews, G., \& Neilson, M. (1988). The Long-Term Outcome of Depressive Illness. The British Journal of Psychiatry, 153(6), 752-757. https://doi.org/10.1192/bjp.153.6.752 
Kim, D.-K., Tolliver, T. J., Huang, S.-J., Martin, B. J., Andrews, A. M., Wichems, C., Holmes, A., Lesch, K.-P., \& Murphy, D. L. (2005). Altered serotonin synthesis, turnover and dynamic regulation in multiple brain regions of mice lacking the serotonin transporter.

Neuropharmacology, 49(6), 798-810. https://doi.org/10.1016/j.neuropharm.2005.08.010

Kim, J., Riggs, K. W., Misri, S., Kent, N., Oberlander, T. F., Grunau, R. E., Fitzgerald, C., \& Rurak, D. W. (2006). Stereoselective disposition of fluoxetine and norfluoxetine during pregnancy and breast-feeding. British Journal of Clinical Pharmacology, 61(2), 155-163. https://doi.org/10.1111/j.1365-2125.2005.02538.x

Kim, W. R., Christian, K., Ming, G.-L., \& Song, H. (2012). Time-dependent involvement of adultborn dentate granule cells in behavior. Behavioural Brain Research, 227(2), 470-479. https://doi.org/10.1016/j.bbr.2011.07.012

Kim, Y.-K., Suh, I.-B., Kim, H., Han, C.-S., Lim, C.-S., Choi, S.-H., \& Licinio, J. (2002). The plasma levels of interleukin-12 in schizophrenia, major depression, and bipolar mania: Effects of psychotropic drugs. Molecular Psychiatry, 7(10), 1107-1114. https://doi.org/10.1038/sj.mp.4001084

Kirkby, L. A., Luongo, F. J., Lee, M. B., Nahum, M., Van Vleet, T. M., Rao, V. R., Dawes, H. E., Chang, E. F., \& Sohal, V. S. (2018). An Amygdala-Hippocampus Subnetwork that Encodes Variation in Human Mood. Cell, 175(6), 1688-1700.e14. https://doi.org/10.1016/j.cell.2018.10.005

Kjelstrup, K. G., Tuvnes, F. A., Steffenach, H.-A., Murison, R., Moser, E. I., \& Moser, M.-B. (2002). Reduced fear expression after lesions of the ventral hippocampus. Proceedings of the National Academy of Sciences, 99(16), 10825-10830. https://doi.org/10.1073/pnas.152112399

Klempin, F., Babu, H., De Pietri Tonel, D., Alarcon, E., Fabel, K., \& Kempermann, G. (2010). Oppositional Effects of Serotonin Receptors 5-HT1a, 2, and 2c in the Regulation of Adult Hippocampal Neurogenesis. Frontiers in Molecular Neuroscience, 3. https://doi.org/10.3389/fnmol.2010.00014

Klempin, F., Beis, D., Mosienko, V., Kempermann, G., Bader, M., \& Alenina, N. (2013). Serotonin Is Required for Exercise-Induced Adult Hippocampal Neurogenesis. Journal of Neuroscience, 33(19), 8270-8275. https://doi.org/10.1523/JNEUROSCI.5855-12.2013 
Kliman, H. J., Quaratella, S. B., Setaro, A. C., Siegman, E. C., Subha, Z. T., Tal, R., Milano, K. M., \& Steck, T. L. (2018). Pathway of Maternal Serotonin to the Human Embryo and Fetus. Endocrinology, 159(4), 1609-1629. https://doi.org/10.1210/en.2017-03025

Kobayashi, K., Ikeda, Y., Sakai, A., Yamasaki, N., Haneda, E., Miyakawa, T., \& Suzuki, H. (2010). Reversal of hippocampal neuronal maturation by serotonergic antidepressants. Proceedings of the National Academy of Sciences, 107(18), 8434-8439. https://doi.org/10.1073/pnas.0912690107

Kocsis, J. H., Gelenberg, A. J., Rothbaum, B., Klein, D. N., Trivedi, M. H., Manber, R., Keller, M. B., Howland, R., \& Thase, M. E. (2008). Chronic forms of major depression are still undertreated in the 21st century: Systematic assessment of 801 patients presenting for treatment. Journal of Affective Disorders, 110(1), 55-61.

https://doi.org/10.1016/j.jad.2008.01.002

Kondo, M., Nakamura, Y., Ishida, Y., \& Shimada, S. (2015). The 5-HT 3 receptor is essential for exercise-induced hippocampal neurogenesis and antidepressant effects. Molecular Psychiatry, 20(11), 1428-1437. https://doi.org/10.1038/mp.2014.153

Kraft, J. B., Slager, S. L., McGrath, P. J., \& Hamilton, S. P. (2005). Sequence Analysis of the Serotonin Transporter and Associations with Antidepressant Response. Biological Psychiatry, 58(5), 374-381. https://doi.org/10.1016/j.biopsych.2005.04.048

Krishnan, V., \& Nestler, E. J. (2008). The molecular neurobiology of depression. Nature, 455(7215), 894-902. https://doi.org/10.1038/nature07455

Kroeze, Y., Dirven, B., Janssen, S., Kröhnke, M., Barte, R. M., Middelman, A., van Bokhoven, H., Zhou, H., \& Homberg, J. R. (2016). Perinatal reduction of functional serotonin transporters results in developmental delay. Neuropharmacology, 109, 96-111. https://doi.org/10.1016/j.neuropharm.2016.05.012

Kronenberg, G., Mosienko, V., Gertz, K., Alenina, N., Hellweg, R., \& Klempin, F. (2016, April). Increased brain-derived neurotrophic factor (BDNF) protein concentrations in mice lacking brain serotonin. European Archives of Psychiatry and Clinical Neuroscience; Eur Arch Psychiatry Clin Neurosci. https://doi.org/10.1007/s00406-015-0611-3

Kronfeld-Schor, N., \& Einat, H. (2012). Circadian rhythms and depression: Human psychopathology and animal models. Neuropharmacology, 62(1), 101-114. https://doi.org/10.1016/j.neuropharm.2011.08.020 
Kronmüller, K.-T., Schröder, J., Köhler, S., Götz, B., Victor, D., Unger, J., Giesel, F., Magnotta, V., Mundt, C., Essig, M., \& Pantel, J. (2009). Hippocampal volume in first episode and recurrent depression. Psychiatry Research: Neuroimaging, 174(1), 62-66.

https://doi.org/10.1016/j.pscychresns.2008.08.001

Kuhn, H. G., Dickinson-Anson, H., \& Gage, F. H. (1996). Neurogenesis in the dentate gyrus of the adult rat: Age-related decrease of neuronal progenitor proliferation. Journal of Neuroscience, 16(6), 2027-2033. https://doi.org/10.1523/JNEUROSCI.16-06-02027.1996

Kuhn, H. Georg, Eisch, A. J., Spalding, K., \& Peterson, D. A. (2016). Detection and Phenotypic Characterization of Adult Neurogenesis. Cold Spring Harbor Perspectives in Biology, 8(3), a025981. https://doi.org/10.1101/cshperspect.a025981

Laplante, D. P., Brunet, A., Schmitz, N., Ciampi, A., \& King, S. (2008). Project Ice Storm: Prenatal Maternal Stress Affects Cognitive and Linguistic Functioning in 51/2-Year-Old Children. Journal of the American Academy of Child \& Adolescent Psychiatry, 47(9), 10631072. https://doi.org/10.1097/CHI.0b013e31817eec80

Lattimore, K. A., Donn, S. M., Kaciroti, N., Kemper, A. R., Neal, C. R., \& Vazquez, D. M. (2005). Selective serotonin reuptake inhibitor (SSRI) use during pregnancy and effects on the fetus and newborn: A meta-analysis. Journal of Perinatology: Official Journal of the California Perinatal Association, 25(9), 595-604. https://doi.org/10.1038/sj.jp.7211352

Lauder, J. (1990). Ontogeny of the serotonergic system in the rat: Serotonin as a developmental signal. Annals of the New York Academy of Sciences, 600, 297-313; discussion 314. https://doi.org/10.1111/j.1749-6632.1990.tb16891.x

Lauder, J. M., \& Bloom, F. E. (1974). Ontogeny of monoamine neurons in the locus coeruleus, raphe nuclei and substantia nigra of the rat. I. Cell differentiation. Journal of Comparative Neurology, 155(4), 469-481. https://doi.org/10.1002/cne.901550407

Lauder, J. M., \& Bloom, F. E. (1975). Ontogeny of monoamine neurons in the locus coeruleus, raphe nuclei and substantia nigra of the rat. Journal of Comparative Neurology, 163(3), 251264. https://doi.org/10.1002/cne.901630302

Lauder, J. M., \& Krebs, H. (1978). Serotonin as a Differentiation Signal in Early Neurogenesis. Developmental Neuroscience, 1(1), 15-30. https://doi.org/10.1159/000112549 
Le François, B., Czesak, M., Steubl, D., \& Albert, P. R. (2008). Transcriptional regulation at a HTR1A polymorphism associated with mental illness. Neuropharmacology, 55(6), 977-985. https://doi.org/10.1016/j.neuropharm.2008.06.046

Le Poul, E., Boni, C., Hanoun, N., Laporte, A.-M., Laaris, N., Chauveau, J., Hamon, M., \& Lanfumey, L. (2000). Differential adaptation of brain 5-HT1A and 5-HT1B receptors and 5HT transporter in rats treated chronically with fluoxetine. Neuropharmacology, 39(1), 110 122. https://doi.org/10.1016/S0028-3908(99)00088-X

Le Poul, E., Laaris, N., Doucet, E., Laporte, A.-M., Hamon, M., \& Lanfumey, L. (1995). Early desensitization of somato-dendritic 5-HT1A autoreceptors in rats treated with fluoxetine or paroxetine. Naunyn-Schmiedeberg's Archives of Pharmacology, 352(2), 141-148. https://doi.org/10.1007/BF00176767

Leader, J. B., \& Klein, D. N. (1996). Social adjustment in dysthymia, double depression and episodic major depression. Journal of Affective Disorders, 37(2), 91-101.

https://doi.org/10.1016/0165-0327(95)00076-3

Lebrand, C., Cases, O., Wehrlé, R., Blakely, R. D., Edwards, R. H., \& Gaspar, P. (1998). Transient developmental expression of monoamine transporters in the rodent forebrain. Journal of Comparative Neurology, 401(4), 506-524. https://doi.org/10.1002/(SICI)10969861(19981130)401:4<506::AID-CNE5>3.0.CO;2-\#

Lee, A. S., \& Murray, R. M. (1988). The Long-Term Outcome of Maudsley Depressives. The British Journal of Psychiatry, 153(6), 741-751. https://doi.org/10.1192/bjp.153.6.741

Lee, J.-H., Kim, H. J., Kim, J. G., Ryu, V., Kim, B.-T., Kang, D.-W., \& Jahng, J. W. (2007). Depressive behaviors and decreased expression of serotonin reuptake transporter in rats that experienced neonatal maternal separation. Neuroscience Research, 58(1), 32-39. https://doi.org/10.1016/j.neures.2007.01.008

Lee, K.-J., Kim, S.-J., Kim, S.-W., Choi, S.-H., Shin, Y.-C., Park, S.-H., Moon, B.-H., Cho, E., Lee, M.-S., Choi, S.-H., Chun, B.-G., \& Shin, K.-H. (2006). Chronic mild stress decreases survival, but not proliferation, of new-born cells in adult rat hippocampus. Experimental \& Molecular Medicine, 38(1), 44-54. https://doi.org/10.1038/emm.2006.6

Lee, S., Jeong, J., Kwak, Y., \& Park, S. K. (2010). Depression research: Where are we now? Molecular Brain, 3(1), 8. https://doi.org/10.1186/1756-6606-3-8 
Leenders, A. G. M., \& Sheng, Z.-H. (2005). Modulation of neurotransmitter release by the second messenger-activated protein kinases: Implications for presynaptic plasticity. Pharmacology \& Therapeutics, 105(1), 69-84. https://doi.org/10.1016/j.pharmthera.2004.10.012

Lemaire, R., Tabet, J. C., Ducoroy, P., Hendra, J. B., Salzet, M., \& Fournier, I. (2006). Solid Ionic Matrixes for Direct Tissue Analysis and MALDI Imaging. Analytical Chemistry, 78(3), 809819. https://doi.org/10.1021/ac0514669

Lemaire, V., Koehl, M., Moal, M. L., \& Abrous, D. N. (2000). Prenatal stress produces learning deficits associated with an inhibition of neurogenesis in the hippocampus. Proceedings of the National Academy of Sciences, 97(20), 11032-11037.

https://doi.org/10.1073/pnas.97.20.11032

Lemonde, S., Turecki, G., Bakish, D., Du, L., Hrdina, P. D., Bown, C. D., Sequeira, A., Kushwaha, N., Morris, S. J., Basak, A., Ou, X.-M., \& Albert, P. R. (2003). Impaired Repression at a 5-Hydroxytryptamine 1A Receptor Gene Polymorphism Associated with Major Depression and Suicide. Journal of Neuroscience, 23(25), 8788-8799. https://doi.org/10.1523/JNEUROSCI.23-25-08788.2003

Leonardo, E. D., \& Hen, R. (2008). Anxiety as a Developmental Disorder.

Neuropsychopharmacology, 33(1), 134-140. https://doi.org/10.1038/sj.npp.1301569

Leopold, J., Popkova, Y., Engel, K. M., \& Schiller, J. (2018). Recent Developments of Useful MALDI Matrices for the Mass Spectrometric Characterization of Lipids. Biomolecules, 8(4). https://doi.org/10.3390/biom8040173

Lesch, K. P., Aulakh, C. S., Wolozin, B. L., Tolliver, T. J., Hill, J. L., \& Murphy, D. L. (1993). Regional brain expression of serotonin transporter mRNA and its regulation by reuptake inhibiting antidepressants. Molecular Brain Research, 17(1), 31-35. https://doi.org/10.1016/0169-328X(93)90069-2

Lesch, K.-P., Bengel, D., Heils, A., Sabol, S. Z., Greenberg, B. D., Petri, S., Benjamin, J., Müller, C. R., Hamer, D. H., \& Murphy, D. L. (1996). Association of Anxiety-Related Traits with a Polymorphism in the Serotonin Transporter Gene Regulatory Region. Science, 274(5292), 1527-1531. https://doi.org/10.1126/science.274.5292.1527

Lesch, K.-P., \& Waider, J. (2012). Serotonin in the Modulation of Neural Plasticity and Networks: Implications for Neurodevelopmental Disorders. Neuron, 76(1), 175-191.

https://doi.org/10.1016/j.neuron.2012.09.013 
Levin, M., Buznikov, G. A., \& Lauder, J. M. (2006). Of Minds and Embryos: Left-Right Asymmetry and the Serotonergic Controls of Pre-Neural Morphogenesis. Developmental Neuroscience, 28(3), 171-185. https://doi.org/10.1159/000091915

Levin, R. L., Heller, W., Mohanty, A., Herrington, J. D., \& Miller, G. A. (2007). Cognitive Deficits in Depression and Functional Specificity of Regional Brain Activity. Cognitive Therapy and Research, 31(2), 211-233. https://doi.org/10.1007/s10608-007-9128-z

Li, N., Lee, B., Liu, R.-J., Banasr, M., Dwyer, J. M., Iwata, M., Li, X.-Y., Aghajanian, G., \& Duman, R. S. (2010). MTOR-Dependent Synapse Formation Underlies the Rapid Antidepressant Effects of NMDA Antagonists. Science, 329(5994), 959-964. https://doi.org/10.1126/science.1190287

Li, S., Zhang, Y., Liu, J., Han, J., Guan, M., Yang, H., Lin, Y., Xiong, S., \& Zhao, Z. (2016). Electrospray deposition device used to precisely control the matrix crystal to improve the performance of MALDI MSI. Scientific Reports, 6. https://doi.org/10.1038/srep37903

Lidgard, R., \& Duncan, M. W. (1995). Utility of matrix-assisted laser desorption/ionization timeof-flight mass spectrometry for the analysis of low molecular weight compounds. Rapid Communications in Mass Spectrometry, 9(2), 128-132. https://doi.org/10.1002/rcm.1290090205

Lidov, H. G. W., \& Molliver, M. E. (1982). Immunohistochemical study of the development of serotonergic neurons in the rat CNS. Brain Research Bulletin, 9(1), 559-604. https://doi.org/10.1016/0361-9230(82)90164-2

Lira, A., Zhou, M., Castanon, N., Ansorge, M. S., Gordon, J. A., Francis, J. H., Bradley-Moore, M., Lira, J., Underwood, M. D., Arango, V., Kung, H. F., Hofer, M. A., Hen, R., \& Gingrich, J. A. (2003). Altered depression-related behaviors and functional changes in the dorsal raphe nucleus of serotonin transporter-deficient mice. Biological Psychiatry, 54(10), 960-971. https://doi.org/10.1016/S0006-3223(03)00696-6

Liu, D., Diorio, J., Day, J. C., Francis, D. D., \& Meaney, M. J. (2000). Maternal care, hippocampal synaptogenesis and cognitive development in rats. Nature Neuroscience, 3(8), 799-806. https://doi.org/10.1038/77702 
Liu, K. Y., Chow, J. M., \& Sherry, C. (2013a). Early Life Obesity and Diabetes: Origins in Pregnancy. 2013. https://doi.org/10.4236/ojemd.2013.31001

Liu, W., Sheng, H., Xu, Y., Liu, Y., Lu, J., \& Ni, X. (2013b). Swimming exercise ameliorates depression-like behavior in chronically stressed rats: Relevant to proinflammatory cytokines and IDO activation. Behavioural Brain Research, 242, 110-116.

https://doi.org/10.1016/j.bbr.2012.12.041

López-Figueroa, A. L., Norton, C. S., López-Figueroa, M. O., Armellini-Dodel, D., Burke, S., Akil, H., López, J. F., \& Watson, S. J. (2004). Serotonin 5-HT1A, 5-HT1B, and 5-HT2A receptor mRNA expression in subjects with major depression, bipolar disorder, and schizophrenia. Biological Psychiatry, 55(3), 225-233.

https://doi.org/10.1016/j.biopsych.2003.09.017

Luo, C., Xu, H., \& Li, X.-M. (2005). Quetiapine reverses the suppression of hippocampal neurogenesis caused by repeated restraint stress. Brain Research, 1063(1), 32-39. https://doi.org/10.1016/j.brainres.2005.09.043

Lüscher, C., Nicoll, R. A., Malenka, R. C., \& Muller, D. (2000). Synaptic plasticity and dynamic modulation of the postsynaptic membrane. Nature Neuroscience, 3(6), 545-550. https://doi.org/10.1038/75714

MacQueen, G. M., Campbell, S., McEwen, B. S., Macdonald, K., Amano, S., Joffe, R. T., Nahmias, C., \& Young, L. T. (2003). Course of illness, hippocampal function, and hippocampal volume in major depression. Proceedings of the National Academy of Sciences, 100(3), 1387-1392. https://doi.org/10.1073/pnas.0337481100

Madrid, J. E., Mandalaywala, T. M., Coyne, S. P., Ahloy-Dallaire, J., Garner, J. P., Barr, C. S., Maestripieri, D., \& Parker, K. J. (2018). Adaptive developmental plasticity in rhesus macaques: The serotonin transporter gene interacts with maternal care to affect juvenile social behaviour. Proceedings of the Royal Society B: Biological Sciences, 285(1881), 20180541. https://doi.org/10.1098/rspb.2018.0541

Magariños, A. M., \& McEwen, B. S. (1995). Stress-induced atrophy of apical dendrites of hippocampal CA3c neurons: Involvement of glucocorticoid secretion and excitatory amino acid receptors. Neuroscience, 69(1), 89-98. https://doi.org/10.1016/0306-4522(95)00259-L 
Magavi, S. S., \& Macklis, J. D. (2008). Identification of Newborn Cells by BrdU Labeling and Immunocytochemistry In Vivo. In L. P. Weiner (Ed.), Neural Stem Cells: Methods and Protocols (pp. 335-343). Humana Press. https://doi.org/10.1007/978-1-59745-133-8_25

Malberg, J. E., Eisch, A. J., Nestler, E. J., \& Duman, R. S. (2000). Chronic Antidepressant Treatment Increases Neurogenesis in Adult Rat Hippocampus. Journal of Neuroscience, 20(24), 9104-9110. https://doi.org/10.1523/JNEUROSCI.20-24-09104.2000

Malison, R. T., Price, L. H., Berman, R., van Dyck, C. H., Pelton, G. H., Carpenter, L., Sanacora, G., Owens, M. J., Nemeroff, C. B., Rajeevan, N., Baldwin, R. M., Seibyl, J. P., Innis, R. B., \& Charney, D. S. (1998). Reduced brain serotonin transporter availability in major depression

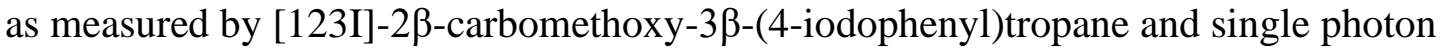
emission computed tomography. Biological Psychiatry, 44(11), 1090-1098. https://doi.org/10.1016/S0006-3223(98)00272-8

Manier, M. L., Spraggins, J. M., Reyzer, M. L., Norris, J. L., \& Caprioli, R. M. (2014). A derivatization and validation strategy for determining the spatial localization of endogenous amine metabolites in tissues using MALDI imaging mass spectrometry. Journal of Mass Spectrometry, 49(8), 665-673. https://doi.org/10.1002/jms.3411

Manji, H. K., Drevets, W. C., \& Charney, D. S. (2001). The cellular neurobiology of depression. Nature Medicine, 7(5), 541-547. https://doi.org/10.1038/87865

Mann, J. J., Huang, Y., Underwood, M. D., Kassir, S. A., Oppenheim, S., Kelly, T. M., Dwork, A. J., \& Arango, V. (2000). A Serotonin Transporter Gene Promoter Polymorphism (5HTTLPR) and Prefrontal Cortical Binding in Major Depression and Suicide. Archives of General Psychiatry, 57(8), 729-738. https://doi.org/10.1001/archpsyc.57.8.729

Mann, M., Hendrickson, R. C., \& Pandey, A. (2001). Analysis of Proteins and Proteomes by Mass Spectrometry. Annual Review of Biochemistry, 70(1), 437-473.

https://doi.org/10.1146/annurev.biochem.70.1.437

Markakis, E. A., \& Gage, F. H. (1999). Adult-generated neurons in the dentate gyrus send axonal projections to field CA3 and are surrounded by synaptic vesicles. The Journal of Comparative Neurology, 406(4), 449-460.

Martinowich, K., \& Lu, B. (2008). Interaction between BDNF and Serotonin: Role in Mood Disorders. Neuropsychopharmacology, 33(1), 73-83. https://doi.org/10.1038/sj.npp.1301571 
Massart, R., Mongeau, R., \& Lanfumey, L. (2012). Beyond the monoaminergic hypothesis: Neuroplasticity and epigenetic changes in a transgenic mouse model of depression. Philosophical Transactions of the Royal Society B: Biological Sciences, 367(1601), 24852494. https://doi.org/10.1098/rstb.2012.0212

Matsukawa, M., Nakadate, K., Ishihara, I., \& Okado, N. (2003). Synaptic loss following depletion of noradrenaline and/or serotonin in the rat visual cortex: A quantitative electron microscopic study. Neuroscience, 122(3), 627-635. https://doi.org/10.1016/j.neuroscience.2003.08.047

Mattson, M. P., Maudsley, S., \& Martin, B. (2004). BDNF and 5-HT: A dynamic duo in agerelated neuronal plasticity and neurodegenerative disorders. Trends in Neurosciences, 27(10), 589-594. https://doi.org/10.1016/j.tins.2004.08.001

Mazer, C., Muneyyirci, J., Taheny, K., Raio, N., Borella, A., \& Whitaker-Azmitia, P. (1997). Serotonin depletion during synaptogenesis leads to decreased synaptic density and learning deficits in the adult rat: A possible model of neurodevelopmental disorders with cognitive deficits. Brain Research, 760(1-2), 68-73. https://doi.org/10.1016/s0006-8993(97)00297-7

McEwen, B. S. (2000). The neurobiology of stress: From serendipity to clinical relevance11Published on the World Wide Web on 22 November 2000. Brain Research, 886(1), 172-189. https://doi.org/10.1016/S0006-8993(00)02950-4

McEwen, B. S., \& Magarinos, A. M. (2001). Stress and hippocampal plasticity: Implications for the pathophysiology of affective disorders. Human Psychopharmacology: Clinical and Experimental, 16(S1), S7-S19. https://doi.org/10.1002/hup.266

McKinnon, M. C., Yucel, K., Nazarov, A., \& MacQueen, G. M. (2009). A meta-analysis examining clinical predictors of hippocampal volume in patients with major depressive disorder. Journal of Psychiatry \& Neuroscience. JPN, 34(1), 41-54.

McKittrick, C. R., Blanchard, D. C., Blanchard, R. J., McEwen, B. S., \& Sakai, R. R. (1995). Serotonin receptor binding in a colony model of chronic social stress. Biological Psychiatry, 37(6), 383-393. https://doi.org/10.1016/0006-3223(94)00152-S

McKittrick, C. R., Magariños, A. M., Blanchard, D. C., Blanchard, R. J., McEwen, B. S., \& Sakai, R. R. (2000). Chronic social stress reduces dendritic arbors in CA3 of hippocampus and decreases binding to serotonin transporter sites. Synapse, 36(2), 85-94. https://doi.org/10.1002/(SICI)1098-2396(200005)36:2<85::AID-SYN1>3.0.CO;2-Y 
Medzihradszky, K. F., Campbell, J. M., Baldwin, M. A., Falick, A. M., Juhasz, P., Vestal, M. L., \& Burlingame, A. L. (2000). The Characteristics of Peptide Collision-Induced Dissociation Using a High-Performance MALDI-TOF/TOF Tandem Mass Spectrometer. Analytical Chemistry, 72(3), 552-558. https://doi.org/10.1021/ac990809y

Melchior, M., Ferrie, J. E., Alexanderson, K., Goldberg, M., Kivimaki, M., Singh-Manoux, A., Vahtera, J., Westerlund, H., Zins, M., \& Head, J. (2010). Does Sickness Absence Due to Psychiatric Disorder Predict Cause-specific Mortality? A 16-Year Follow-up of the GAZEL Occupational Cohort Study. American Journal of Epidemiology, 172(6), 700-707. https://doi.org/10.1093/aje/kwq186

Mendez-David, I., David, D. J., Darcet, F., Wu, M. V., Kerdine-Römer, S., Gardier, A. M., \& Hen, R. (2014). Rapid Anxiolytic Effects of a 5-HT 4 Receptor Agonist Are Mediated by a Neurogenesis-Independent Mechanism. Neuropsychopharmacology, 39(6), 1366-1378. https://doi.org/10.1038/npp.2013.332

Mendoza, J. E., \& Foundas, A. L. (2008). The Limbic System/Hypothalamus. In J. E. Mendoza \& A. L. Foundas (Eds.), Clinical Neuroanatomy: A Neurobehavioral Approach (pp. 213-269). Springer. https://doi.org/10.1007/978-0-387-36601-2_8

Míguez, J. M., Aldegunde, M., Paz-Valiñas, L., Recio, J., \& Sánchez-Barceló, E. (1999). Selective changes in the contents of noradrenaline, dopamine and serotonin in rat brain areas during aging. Journal of Neural Transmission, 106(11), 1089-1098.

https://doi.org/10.1007/s007020050225

Mileva-Seitz, V., Kennedy, J., Atkinson, L., Steiner, M., Levitan, R., Matthews, S. G., Meaney, M. J., Sokolowski, M. B., \& Fleming, A. S. (2011). Serotonin transporter allelic variation in mothers predicts maternal sensitivity, behavior and attitudes toward 6-month-old infants. Genes, Brain and Behavior, 10(3), 325-333. https://doi.org/10.1111/j.1601$\underline{183 X .2010 .00671 . X}$

Mill, J., \& Petronis, A. (2007). Molecular studies of major depressive disorder: The epigenetic perspective. Molecular Psychiatry, 12(9), 799-814. https://doi.org/10.1038/sj.mp.4001992

Miller, R., Wankerl, M., Stalder, T., Kirschbaum, C., \& Alexander, N. (2013). The serotonin transporter gene-linked polymorphic region (5-HTTLPR) and cortisol stress reactivity: A meta-analysis. Molecular Psychiatry, 18(9), 1018-1024. https://doi.org/10.1038/mp.2012.124 
Ming, G., \& Song, H. (2011). Adult Neurogenesis in the Mammalian Brain: Significant Answers and Significant Questions. Neuron, 70(4), 687-702.

https://doi.org/10.1016/j.neuron.2011.05.001

Mintz, J., Mintz, L. I., Arruda, M. J., \& Hwang, S. S. (1992). Treatments of Depression and the Functional Capacity to Work. Archives of General Psychiatry, 49(10), 761-768. https://doi.org/10.1001/archpsyc.1992.01820100005001

Mirescu, C., \& Gould, E. (2006). Stress and adult neurogenesis. Hippocampus, 16(3), 233-238. https://doi.org/10.1002/hipo.20155

Mitra, R., Sundlass, K., Parker, K. J., Schatzberg, A. F., \& Lyons, D. M. (2006). Social stressrelated behavior affects hippocampal cell proliferation in mice. Physiology \& Behavior, 89(2), 123-127. https://doi.org/10.1016/j.physbeh.2006.05.047

Mohapel, P., Leanza, G., Kokaia, M., \& Lindvall, O. (2005). Forebrain acetylcholine regulates adult hippocampal neurogenesis and learning. Neurobiology of Aging, 26(6), 939-946. https://doi.org/10.1016/j.neurobiolaging.2004.07.015

Mojtabai, R., Olfson, M., \& Han, B. (2016). National Trends in the Prevalence and Treatment of Depression in Adolescents and Young Adults. Pediatrics, 138(6).

https://doi.org/10.1542/peds.2016-1878

Montañez, S., Owens, W. A., Gould, G. G., Murphy, D. L., \& Daws, L. C. (2003). Exaggerated effect of fluvoxamine in heterozygote serotonin transporter knockout mice. Journal of Neurochemistry, 86(1), 210-219. https://doi.org/10.1046/j.1471-4159.2003.01836.x

Moreno-Jiménez, E. P., Flor-García, M., Terreros-Roncal, J., Rábano, A., Cafini, F., PallasBazarra, N., Ávila, J., \& Llorens-Martín, M. (2019). Adult hippocampal neurogenesis is abundant in neurologically healthy subjects and drops sharply in patients with Alzheimer's disease. Nature Medicine, 25(4), 554-560. https://doi.org/10.1038/s41591-019-0375-9

Moret, C., \& Briley, M. (2000). The possible role of 5-HT(1B/D) receptors in psychiatric disorders and their potential as a target for therapy. European Journal of Pharmacology, 404(1-2), 1-12. https://doi.org/10.1016/s0014-2999(00)00581-1

Morris, J. S., Smith, K. A., Cowen, P. J., Friston, K. J., \& Dolan, R. J. (1999). Covariation of Activity in Habenula and Dorsal Raphé Nuclei Following Tryptophan Depletion.

NeuroImage, 10(2), 163-172. https://doi.org/10.1006/nimg.1999.0455 
Morris, R. G. M., Garrud, P., Rawlins, J. N. P., \& O’Keefe, J. (1982). Place navigation impaired in rats with hippocampal lesions. Nature, 297(5868), 681-683.

https://doi.org/10.1038/297681a0

Moser, M. B., Moser, E. I., Forrest, E., Andersen, P., \& Morris, R. G. (1995). Spatial learning with a minislab in the dorsal hippocampus. Proceedings of the National Academy of Sciences, 92(21), 9697-9701. https://doi.org/10.1073/pnas.92.21.9697

Moser, M.-B., \& Moser, E. I. (1998). Functional differentiation in the hippocampus.

Hippocampus, 8(6), 608-619. https://doi.org/10.1002/(SICI)1098-1063(1998)8:6<608::AID$\underline{\mathrm{HIPO}}>3.0 . \mathrm{CO} ; 2-7$

Mueller, T. I., Leon, A. C., Keller, M. B., Solomon, D. A., Endicott, J., Coryell, W., Warshaw, M., \& Maser, J. D. (1999). Recurrence After Recovery From Major Depressive Disorder During 15 Years of Observational Follow-Up. American Journal of Psychiatry, 156(7), 1000-1006. https://doi.org/10.1176/ajp.156.7.1000

Mulder, E. J. H., Ververs, F. F., de Heus, R., \& Visser, G. H. A. (2011). Selective Serotonin Reuptake Inhibitors Affect Neurobehavioral Development in the Human Fetus. Neuropsychopharmacology, 36(10), 1961-1971. https://doi.org/10.1038/npp.2011.67

Muller, C. L., Anacker, A. M., Rogers, T. D., Goeden, N., Keller, E. H., Forsberg, C. G., Kerr, T. M., Wender, C. L., Anderson, G. M., Stanwood, G. D., Blakely, R. D., Bonnin, A., \& Veenstra-VanderWeele, J. (2017). Impact of Maternal Serotonin Transporter Genotype on Placental Serotonin, Fetal Forebrain Serotonin, and Neurodevelopment. Neuropsychopharmacology, 42(2), 427-436. https://doi.org/10.1038/npp.2016.166

Munafò, M. R., Brown, S. M., \& Hariri, A. R. (2008). Serotonin Transporter (5-HTTLPR) Genotype and Amygdala Activation: A Meta-Analysis. Biological Psychiatry, 63(9), 852857. https://doi.org/10.1016/j.biopsych.2007.08.016

Murphy, D. L., Lerner, A., Rudnick, G., \& Lesch, K.-P. (2004). Serotonin Transporter: Gene, Genetic Disorders, and Pharmacogenetics. Molecular Interventions, 4(2), 109. https://doi.org/10.1124/mi.4.2.8

Nagashima, T., DeArmond, S. J., Murovic, J., \& Hoshino, T. (1985). Immunocytochemical demonstration of S-phase cells by anti-bromodeoxyuridine monoclonal antibody in human brain tumor tissues. Acta Neuropathologica, 67(1), 155-159.

https://doi.org/10.1007/BF00688136 
Nakano-Kobayashi, A., Awaya, T., Kii, I., Sumida, Y., Okuno, Y., Yoshida, S., Sumida, T., Inoue, H., Hosoya, T., \& Hagiwara, M. (2017). Prenatal neurogenesis induction therapy normalizes brain structure and function in Down syndrome mice. Proceedings of the National Academy of Sciences, 114(38), 10268-10273. https://doi.org/10.1073/pnas.1704143114

Narboux-Nême, N., Pavone, L. M., Avallone, L., Zhuang, X., \& Gaspar, P. (2008). Serotonin transporter transgenic (SERTcre) mouse line reveals developmental targets of serotonin specific reuptake inhibitors (SSRIs). Neuropharmacology, 55(6), 994-1005. https://doi.org/10.1016/j.neuropharm.2008.08.020

Nasir, N., \& Khan, A. (2011). Effects of stress-induced acute depression and antidepressant drugs on CA3 region of hippocampus of albino rats. Current Opinion in Neurology, 2, 31-34.

Neumaier, J. F., Petty, F., Kramer, G. L., Szot, P., \& Hamblin, M. W. (1997). Learned helplessness increases 5-hydroxytryptamine1B receptor mRNA levels in the rat dorsal raphe nucleus. Biological Psychiatry, 41(6), 668-674. https://doi.org/10.1016/S0006$\underline{3223(96) 00114-X}$

Neumeister, A. (2003). Tryptophan depletion, serotonin, and depression: Where do we stand? Psychopharmacology Bulletin, 37(4), 99-115.

Neumeister, A., Nugent, A. C., Waldeck, T., Geraci, M., Schwarz, M., Bonne, O., Bain, E. E., Luckenbaugh, D. A., Herscovitch, P., Charney, D. S., \& Drevets, W. C. (2004). Neural and Behavioral Responses to Tryptophan Depletion in UnmedicatedPatients With Remitted Major Depressive Disorder and Controls. Archives of General Psychiatry, 61(8), 765-773. https://doi.org/10.1001/archpsyc.61.8.765

Nguyen, P. V., \& Kandel, E. R. (1996). A Macromolecular Synthesis-Dependent Late Phase of Long-Term Potentiation Requiring cAMP in the Medial Perforant Pathway of Rat Hippocampal Slices. Journal of Neuroscience, 16(10), 3189-3198. https://doi.org/10.1523/JNEUROSCI.16-10-03189.1996

Ngwenya, L. B., Peters, A., \& Rosene, D. L. (2005). Light and Electron Microscopic Immunohistochemical Detection of Bromodeoxyuridine-labeled Cells in the Brain: Different Fixation and Processing Protocols. Journal of Histochemistry \& Cytochemistry, 53(7), 821832. https://doi.org/10.1369/jhc.4A6605.2005 
Niitsu, Y., Hatnada, S., Hamaguchi, K., Mikuni, M., \& Okado, N. (1995). Regulation of synapse density by 5-HT2A receptor agonist and antagonist in the spinal cord of chicken embryo. Neuroscience Letters, 195(3), 159-162. https://doi.org/10.1016/0304-3940(95)11805-7

Nishi, M., \& Azmitia, E. C. (1999). Agonist- and antagonist-induced plasticity of rat 5-HT1A receptor in hippocampal cell culture. Synapse, 31(3), 186-195. https://doi.org/10.1002/(SICI)1098-2396(19990301)31:3<186::AID-SYN3>3.0.CO;2-O

Noorlander, C. W., Ververs, F. F. T., Nikkels, P. G. J., Echteld, C. J. A. van, Visser, G. H. A., \& Smidt, M. P. (2008). Modulation of Serotonin Transporter Function during Fetal Development Causes Dilated Heart Cardiomyopathy and Lifelong Behavioral Abnormalities. PLOS ONE, 3(7), e2782. https://doi.org/10.1371/journal.pone.0002782

Norris, J. L., \& Caprioli, R. M. (2013). Analysis of Tissue Specimens by Matrix-Assisted Laser Desorption/Ionization Imaging Mass Spectrometry in Biological and Clinical Research. Chemical Reviews, 113(4), 2309-2342. https://doi.org/10.1021/cr3004295

Novkovic, T., Mittmann, T., \& Manahan-Vaughan, D. (2015). BDNF contributes to the facilitation of hippocampal synaptic plasticity and learning enabled by environmental enrichment. Hippocampus, 25(1), 1-15. https://doi.org/10.1002/hipo.22342

Nulman, I., Koren, G., Rovet, J., Barrera, M., Pulver, A., Streiner, D., \& Feldman, B. (2012). Neurodevelopment of Children Following Prenatal Exposure to Venlafaxine, Selective Serotonin Reuptake Inhibitors, or Untreated Maternal Depression. American Journal of Psychiatry, 169(11), 1165-1174. https://doi.org/10.1176/appi.ajp.2012.11111721

Oberlander, T. F., Gingrich, J. A., \& Ansorge, M. S. (2009). Sustained Neurobehavioral Effects of Exposure to SSRI Antidepressants During Development: Molecular to Clinical Evidence. Clinical Pharmacology \& Therapeutics, 86(6), 672-677. https://doi.org/10.1038/clpt.2009.201

Oberlander, Tim F., Papsdorf, M., Brain, U. M., Misri, S., Ross, C., \& Grunau, R. E. (2010). Prenatal Effects of Selective Serotonin Reuptake Inhibitor Antidepressants, Serotonin Transporter Promoter Genotype (SLC6A4), and Maternal Mood on Child Behavior at 3 Years of Age. Archives of Pediatrics \& Adolescent Medicine, 164(5), 444-451. https://doi.org/10.1001/archpediatrics.2010.51

Ogilvie, A. D., Battersby, S., Fink, G., Harmar, A. J., Goodwin, G. M., Bubb, V. J., \& Smith, C. A. D. (1996). Polymorphism in serotonin transporter gene associated with susceptibility to 
major depression. The Lancet, 347(9003), 731-733. https://doi.org/10.1016/S0140$\underline{6736(96) 90079-3}$

Olivier, J. D. A., Van Der Hart, M. G. C., Van Swelm, R. P. L., Dederen, P. J., Homberg, J. R., Cremers, T., Deen, P. M. T., Cuppen, E., Cools, A. R., \& Ellenbroek, B. A. (2008). A study in male and female 5-HT transporter knockout rats: An animal model for anxiety and depression disorders. Neuroscience, 152(3), 573-584.

https://doi.org/10.1016/j.neuroscience.2007.12.032

Olson, A. K., Eadie, B. D., Ernst, C., \& Christie, B. R. (2006). Environmental enrichment and voluntary exercise massively increase neurogenesis in the adult hippocampus via dissociable pathways. Hippocampus, 16(3), 250-260. https://doi.org/10.1002/hipo.20157

Olson, L., Boréus, L. O., \& Seiger, A. (1973). Histochemical demonstration and mapping of 5hydroxytryptamine- and catecholamine-containing neuron systems in the human fetal brain. Zeitschrift Fur Anatomie Und Entwicklungsgeschichte, 139(3), 259-282. https://doi.org/10.1007/BF00519968

Olson, Lars, \& Seiger, Å. (1972). Early prenatal ontogeny of central monoamine neurons in the rat: Fluorescence histochemical observations. Zeitschrift Für Anatomie Und Entwicklungsgeschichte, 137(3), 301-316. https://doi.org/10.1007/BF00519099

Ösby, U., Brandt, L., Correia, N., Ekbom, A., \& Sparén, P. (2001). Excess Mortality in Bipolar and Unipolar Disorder in Sweden. Archives of General Psychiatry, 58(9), 844-850. https://doi.org/10.1001/archpsyc.58.9.844

Overstreet, D. H., Friedman, E., Mathé, A. A., \& Yadid, G. (2005). The Flinders Sensitive Line rat: A selectively bred putative animal model of depression. Neuroscience \& Biobehavioral Reviews, 29(4), 739-759. https://doi.org/10.1016/j.neubiorev.2005.03.015

Owens, M. J., \& Nemeroff, C. B. (1994). Role of serotonin in the pathophysiology of depression: Focus on the serotonin transporter. Clinical Chemistry, 40(2), 288-295. https://doi.org/10.1093/clinchem/40.2.288

Paizanis, E., Hamon, M., \& Lanfumey, L. (2007, May 14). Hippocampal Neurogenesis, Depressive Disorders, and Antidepressant Therapy [Review Article]. Neural Plasticity; Hindawi. https://doi.org/10.1155/2007/73754 
Palanisamy, S., Huang, S., Zhao, H., Zhu, D., \& Zhang, X. (2019). In-situ derivatization of Au nanoclusters via aurophilic interaction of triphenylphosphine gold (I) salt with neurotransmitters and its rapid MALDI-TOF-MS detection in mice brain tissue extracts. Journal of Materials Chemistry B. https://doi.org/10.1039/C9TB01800J

Parihar, V. K., Hattiangady, B., Shuai, B., \& Shetty, A. K. (2013). Mood and Memory Deficits in a Model of Gulf War Illness Are Linked with Reduced Neurogenesis, Partial Neuron Loss, and Mild Inflammation in the Hippocampus. Neuropsychopharmacology, 38(12), 2348-2362. https://doi.org/10.1038/npp.2013.158

Pascual-Brazo, J., Castro, E., Díaz, Á., Valdizán, E. M., Pilar-Cuéllar, F., Vidal, R., Treceño, B., \& Pazos, Á. (2012). Modulation of neuroplasticity pathways and antidepressant-like behavioural responses following the short-term ( 3 and 7 days) administration of the 5-HT4 receptor agonist RS67333. International Journal of Neuropsychopharmacology, 15(5), 631643. https://doi.org/10.1017/S1461145711000782

Pearson, R. M., Evans, J., Kounali, D., Lewis, G., Heron, J., Ramchandani, P. G., O’Connor, T. G., \& Stein, A. (2013). Maternal Depression During Pregnancy and the Postnatal Period: Risks and Possible Mechanisms for Offspring Depression at Age 18 Years. JAMA Psychiatry, 70(12), 1312-1319. https://doi.org/10.1001/jamapsychiatry.2013.2163

Perera, T. D., Coplan, J. D., Lisanby, S. H., Lipira, C. M., Arif, M., Carpio, C., Spitzer, G., Santarelli, L., Scharf, B., Hen, R., Rosoklija, G., Sackeim, H. A., \& Dwork, A. J. (2007). Antidepressant-Induced Neurogenesis in the Hippocampus of Adult Nonhuman Primates. Journal of Neuroscience, 27(18), 4894-4901. https://doi.org/10.1523/JNEUROSCI.0237$\underline{07.2007}$

Perlman, G., Simmons, A. N., Wu, J., Hahn, K. S., Tapert, S. F., Max, J. E., Paulus, M. P., Brown, G. G., Frank, G. K., Campbell-Sills, L., \& Yang, T. T. (2012). Amygdala response and functional connectivity during emotion regulation: A study of 14 depressed adolescents. Journal of Affective Disorders, 139(1), 75-84. https://doi.org/10.1016/j.jad.2012.01.044

Piatti, V. C., Davies-Sala, M. G., Espósito, M. S., Mongiat, L. A., Trinchero, M. F., \& Schinder, A. F. (2011). The Timing for Neuronal Maturation in the Adult Hippocampus Is Modulated by Local Network Activity. Journal of Neuroscience, 31(21), 7715-7728. https://doi.org/10.1523/JNEUROSCI.1380-11.2011 
Pittenger, C., \& Duman, R. S. (2008). Stress, Depression, and Neuroplasticity: A Convergence of Mechanisms. Neuropsychopharmacology, 33(1), 88-109.

https://doi.org/10.1038/sj.npp.1301574

Pompeiano, M., Palacios, J. M., \& Mengod, G. (1992). Distribution and cellular localization of mRNA coding for 5-HT1A receptor in the rat brain: Correlation with receptor binding. Journal of Neuroscience, 12(2), 440-453. https://doi.org/10.1523/JNEUROSCI.12-02$\underline{00440.1992}$

Quintana, J. (1992). Platelet serotonin and plasma tryptophan decreases in endogenous depression. Clinical, therapeutic, and biological correlations. Journal of Affective Disorders, 24(2), 5562. https://doi.org/10.1016/0165-0327(92)90019-3

Radley, J. J., \& Jacobs, B. L. (2002). 5-HT1A receptor antagonist administration decreases cell proliferation in the dentate gyrus. Brain Research, 955(1), 264-267. https://doi.org/10.1016/S0006-8993(02)03477-7

Rezvani, A. H., Parsian, A., \& Overstreet, D. H. (2002). The Fawn-Hooded (FH/Wjd) rat: A genetic animal model of comorbid depression and alcoholism. Psychiatric Genetics, 12(1), 116.

Riad, M., Emerit, M. B., \& Hamon, M. (1994). Neurotrophic effects of ipsapirone and other 5HT1A receptor agonists on septal cholinergic neurons in culture. Brain Research. Developmental Brain Research, 82(1-2), 245-258. https://doi.org/10.1016/0165$\underline{3806(94) 90167-8}$

Riad, M., Garcia, S., Watkins, K. C., Jodoin, N., Doucet, E., Langlois, X., el Mestikawy, S., Hamon, M., \& Descarries, L. (2000). Somatodendritic localization of 5-HT1A and preterminal axonal localization of 5-HT1B serotonin receptors in adult rat brain. The Journal of Comparative Neurology, 417(2), 181-194.

Ribeiro, J. D., Huang, X., Fox, K. R., \& Franklin, J. C. (2018). Depression and hopelessness as risk factors for suicide ideation, attempts and death: Meta-analysis of longitudinal studies. British Journal of Psychiatry, 212(5), 279-286. https://doi.org/10.1192/bjp.2018.27

Richardson-Jones, J. W., Craige, C. P., Nguyen, T. H., Kung, H. F., Gardier, A. M., Dranovsky, A., David, D. J., Guiard, B. P., Beck, S. G., Hen, R., \& Leonardo, E. D. (2011). Serotonin-1A Autoreceptors Are Necessary and Sufficient for the Normal Formation of Circuits Underlying 
Innate Anxiety. Journal of Neuroscience, 31(16), 6008-6018.

https://doi.org/10.1523/JNEUROSCI.5836-10.2011

Rock, P. L., Roiser, J. P., Riedel, W. J., \& Blackwell, A. D. (2014). Cognitive impairment in depression: A systematic review and meta-analysis. Psychological Medicine, 44(10), 20292040. https://doi.org/10.1017/S0033291713002535

Rogers, M. A., Kasai, K., Koji, M., Fukuda, R., Iwanami, A., Nakagome, K., Fukuda, M., \& Kato, N. (2004). Executive and prefrontal dysfunction in unipolar depression: A review of neuropsychological and imaging evidence. Neuroscience Research, 50(1), 1-11. https://doi.org/10.1016/j.neures.2004.05.003

Roozendaal, B., Phillips, R. G., Power, A. E., Brooke, S. M., Sapolsky, R. M., \& McGaugh, J. L. (2001). Memory retrieval impairment induced by hippocampal CA3 lesions is blocked by adrenocortical suppression. Nature Neuroscience, 4(12), 1169-1171.

https://doi.org/10.1038/nn766

Rosenfeld, C. S. (2020). Placental serotonin signaling, pregnancy outcomes, and regulation of fetal brain development $\dagger$. Biology of Reproduction, 102(3), 532-538.

https://doi.org/10.1093/biolre/ioz204

Rosenthal, N. E., Mazzanti, C. M., Barnett, R. L., Hardin, T. A., Turner, E. H., Lam, G. K., Ozaki, N., \& Goldman, D. (1998). Role of serotonin transporter promoter repeat length polymorphism (5-HTTLPR) in seasonality and seasonal affective disorder. Molecular Psychiatry, 3(2), 175-177. https://doi.org/10.1038/sj.mp.4000360

Roy, A., Jong, J. D., \& Linnoila, M. (1989). Cerebrospinal Fluid Monoamine Metabolites and Suicidal Behavior in Depressed Patients: A 5-Year Follow-up Study. Archives of General Psychiatry, 46(7), 609-612. https://doi.org/10.1001/archpsyc.1989.01810070035005

Rudnick, G. (1977). Active transport of 5-hydroxytryptamine by plasma membrane vesicles isolated from human blood platelets. Journal of Biological Chemistry, 252(7), 2170-2174. https://doi.org/10.1016/S0021-9258(17)40535-7

Rudnick, Gary, \& Clark, J. (1993). From synapse to vesicle: The reuptake and storage of biogenic amine neurotransmitters. Biochimica et Biophysica Acta (BBA) - Bioenergetics, 1144(3), 249-263. https://doi.org/10.1016/0005-2728(93)90109-S 
Ruhé, H. G., Mason, N. S., \& Schene, A. H. (2007). Mood is indirectly related to serotonin, norepinephrine and dopamine levels in humans: A meta-analysis of monoamine depletion studies. Molecular Psychiatry, 12(4), 331-359. https://doi.org/10.1038/sj.mp.4001949

Sahay, A., \& Hen, R. (2007). Adult hippocampal neurogenesis in depression. Nature Neuroscience, 10(9), 1110-1115. https://doi.org/10.1038/nn1969

Sahay, A., Scobie, K. N., Hill, A. S., O’Carroll, C. M., Kheirbek, M. A., Burghardt, N. S., Fenton, A. A., Dranovsky, A., \& Hen, R. (2011). Increasing adult hippocampal neurogenesis is sufficient to improve pattern separation. Nature, 472(7344), 466-470. https://doi.org/10.1038/nature09817

Salichon, N., Gaspar, P., Upton, A. L., Picaud, S., Hanoun, N., Hamon, M., De Maeyer, E., Murphy, D. L., Mossner, R., Lesch, K. P., Hen, R., \& Seif, I. (2001). Excessive activation of serotonin (5-HT) 1B receptors disrupts the formation of sensory maps in monoamine oxidase a and 5-ht transporter knock-out mice. The Journal of Neuroscience: The Official Journal of the Society for Neuroscience, 21(3), 884-896.

Salomon, R. M., Kennedy, J. S., Johnson, B. W., Schmidt, D. E., Kwentus, J., Gwirtsman, H. E., \& Ebert, M. H. (2003). Association of a Critical CSF Tryptophan Threshold Level with Depressive Relapse. Neuropsychopharmacology, 28(5), 956-960. https://doi.org/10.1038/sj.npp.1300098

Santarelli, L., Saxe, M., Gross, C., Surget, A., Battaglia, F., Dulawa, S., Weisstaub, N., Lee, J., Duman, R., Arancio, O., Belzung, C., \& Hen, R. (2003). Requirement of Hippocampal Neurogenesis for the Behavioral Effects of Antidepressants. Science, 301(5634), 805-809. https://doi.org/10.1126/science.1083328

Sapolsky, R. M., Krey, L. C., \& McEwen, B. S. (1984). Glucocorticoid-sensitive hippocampal neurons are involved in terminating the adrenocortical stress response. Proceedings of the National Academy of Sciences, 81(19), 6174-6177. https://doi.org/10.1073/pnas.81.19.6174

Sapolsky, R. M., Uno, H., Rebert, C. S., \& Finch, C. E. (1990). Hippocampal damage associated with prolonged glucocorticoid exposure in primates. Journal of Neuroscience, 10(9), 28972902. https://doi.org/10.1523/JNEUROSCI.10-09-02897.1990

Sapolsky, Robert M. (2000). Glucocorticoids and Hippocampal Atrophy in Neuropsychiatric Disorders. Archives of General Psychiatry, 57(10), 925-935.

https://doi.org/10.1001/archpsyc.57.10.925 
Sapolsky, Robert M. (2004). Is impaired neurogenesis relevant to the affective symptoms of depression? Biological Psychiatry, 56(3), 137-139.

https://doi.org/10.1016/j.biopsych.2004.04.012

Savitz, J., Lucki, I., \& Drevets, W. C. (2009). 5-HT1A receptor function in major depressive disorder. Progress in Neurobiology, 88(1), 17-31. https://doi.org/10.1016/j.pneurobio.2009.01.009

Saxe, M. D., Battaglia, F., Wang, J.-W., Malleret, G., David, D. J., Monckton, J. E., Garcia, A. D. R., Sofroniew, M. V., Kandel, E. R., Santarelli, L., Hen, R., \& Drew, M. R. (2006). Ablation of hippocampal neurogenesis impairs contextual fear conditioning and synaptic plasticity in the dentate gyrus. Proceedings of the National Academy of Sciences, 103(46), 17501-17506. https://doi.org/10.1073/pnas.0607207103

Scharfman, H., Goodman, J., Macleod, A., Phani, S., Antonelli, C., \& Croll, S. (2005). Increased neurogenesis and the ectopic granule cells after intrahippocampal BDNF infusion in adult rats. Experimental Neurology, 192(2), 348-356.

https://doi.org/10.1016/j.expneurol.2004.11.016

Schipper, P., Kiliaan, A. J., \& Homberg, J. R. (2011). A mixed polyunsaturated fatty acid diet normalizes hippocampal neurogenesis and reduces anxiety in serotonin transporter knockout rats. Behavioural Pharmacology, 22(4), 324-334.

https://doi.org/10.1097/FBP.0b013e328347881b

Schoenfeld, T. J., \& Gould, E. (2012). Stress, stress hormones, and adult neurogenesis. Experimental Neurology, 233(1), 12-21. https://doi.org/10.1016/j.expneurol.2011.01.008

Schubert, K. O., Weiland, F., Baune, B. T., \& Hoffmann, P. (2016). The use of MALDI-MSI in the investigation of psychiatric and neurodegenerative disorders: A review. Proteomics, 16(11-12), 1747-1758. https://doi.org/10.1002/pmic.201500460

Schwamborn, K., \& Caprioli, R. M. (2010). Molecular imaging by mass spectrometry_Looking beyond classical histology. Nature Reviews Cancer, 10(9), 639-646.

https://doi.org/10.1038/nrc2917

Schwartz, S. A., Reyzer, M. L., \& Caprioli, R. M. (2003). Direct tissue analysis using matrixassisted laser desorption/ionization mass spectrometry: Practical aspects of sample preparation. Journal of Mass Spectrometry: JMS, 38(7), 699-708.

https://doi.org/10.1002/jms.505 
Seiger, A., \& Olson, L. (1973). Late prenatal ontogeny of central monoamine neurons in the rat: Fluorescence histochemical observations. Zeitschrift Für Anatomie Und Entwicklungsgeschichte, 140(3), 281-318. https://doi.org/10.1007/BF00525058

Seress, L., Ábrahám, H., Tornóczky, T., \& Kosztolányi, G. (2001). Cell formation in the human hippocampal formation from mid-gestation to the late postnatal period. Neuroscience, 105(4), 831-843. https://doi.org/10.1016/S0306-4522(01)00156-7

Serretti, A., Cusin, C., Rossini, D., Artioli, P., Dotoli, D., \& Zanardi, R. (2004). Further evidence of a combined effect of SERTPR and TPH on SSRIs response in mood disorders. American Journal of Medical Genetics Part B: Neuropsychiatric Genetics, 129B(1), 36-40. https://doi.org/10.1002/ajmg.b.30027

Serretti, A., Kato, M., De Ronchi, D., \& Kinoshita, T. (2007). Meta-analysis of serotonin transporter gene promoter polymorphism (5-HTTLPR) association with selective serotonin reuptake inhibitor efficacy in depressed patients. Molecular Psychiatry, 12(3), 247-257. https://doi.org/10.1038/sj.mp.4001926

Setou, M. (2010). Imaging Mass Spectrometry: Protocols for Mass Microscopy. Springer Science \& Business Media.

Shadrina, M., Bondarenko, E. A., \& Slominsky, P. A. (2018). Genetics Factors in Major Depression Disease. Frontiers in Psychiatry, 9. https://doi.org/10.3389/fpsyt.2018.00334

Shah, R., Courtiol, E., Castellanos, F. X., \& Teixeira, C. M. (2018). Abnormal Serotonin Levels During Perinatal Development Lead to Behavioral Deficits in Adulthood. Frontiers in Behavioral Neuroscience, 12. https://doi.org/10.3389/fnbeh.2018.00114

Shariatgorji, M., Nilsson, A., Goodwin, R. J. A., Källback, P., Schintu, N., Zhang, X., Crossman, A. R., Bezard, E., Svenningsson, P., \& Andren, P. E. (2014). Direct Targeted Quantitative Molecular Imaging of Neurotransmitters in Brain Tissue Sections. Neuron, 84(4), 697-707. https://doi.org/10.1016/j.neuron.2014.10.011

Shariatgorji, M., Nilsson, A., Källback, P., Karlsson, O., Zhang, X., Svenningsson, P., \& Andren, P. E. (2015). Pyrylium Salts as Reactive Matrices for MALDI-MS Imaging of Biologically Active Primary Amines. Journal of The American Society for Mass Spectrometry, 26(6), 934-939. https://doi.org/10.1007/s13361-015-1119-9 
Sheline, Y. I., Wang, P. W., Gado, M. H., Csernansky, J. G., \& Vannier, M. W. (1996). Hippocampal atrophy in recurrent major depression. Proceedings of the National Academy of Sciences, 93(9), 3908-3913. https://doi.org/10.1073/pnas.93.9.3908

Sheline, Y. I., Sanghavi, M., Mintun, M. A., \& Gado, M. H. (1999). Depression Duration But Not Age Predicts Hippocampal Volume Loss in Medically Healthy Women with Recurrent Major Depression. Journal of Neuroscience, 19(12), 5034-5043. https://doi.org/10.1523/JNEUROSCI.19-12-05034.1999

Shemer, A. V., Azmitia, E. C., \& Whitaker-Azmitia, P. M. (1991). Dose-related effects of prenatal 5-methoxytryptamine (5-MT) on development of serotonin terminal density and behavior. Developmental Brain Research, 59(1), 59-63. https://doi.org/10.1016/0165-3806(91)90029-I

Sheng, M., \& Kim, M. J. (2002). Postsynaptic Signaling and Plasticity Mechanisms. Science, 298(5594), 776-780. https://doi.org/10.1126/science.1075333

Shihabuddin, L. S., Horner, P. J., Ray, J., \& Gage, F. H. (2000). Adult Spinal Cord Stem Cells Generate Neurons after Transplantation in the Adult Dentate Gyrus. Journal of Neuroscience, 20(23), 8727-8735. https://doi.org/10.1523/JNEUROSCI.20-23-08727.2000

Shors, T. J., Miesegaes, G., Beylin, A., Zhao, M., Rydel, T., \& Gould, E. (2001). Neurogenesis in the adult is involved in the formation of trace memories. Nature, 410(6826), 372-376. https://doi.org/10.1038/35066584

Shors, T. J., Townsend, D. A., Zhao, M., Kozorovitskiy, Y., \& Gould, E. (2002). Neurogenesis may relate to some but not all types of hippocampal-dependent learning. Hippocampus, 12(5), 578-584. https://doi.org/10.1002/hipo.10103

Sidman, R. L., \& Rakic, P. (1973). Neuronal migration, with special reference to developing human brain: A review. Brain Research, 62(1), 1-35. https://doi.org/10.1016/0006$\underline{8993(73) 90617-3}$

Sikich, L., Hickok, J. M., \& Todd, R. D. (1990). 5-HT1A receptors control neurite branching during development. Developmental Brain Research, 56(2), 269-274.

https://doi.org/10.1016/0165-3806(90)90092-D

Silva, R., Mesquita, A. R., Bessa, J., Sousa, J. C., Sotiropoulos, I., Leão, P., Almeida, O. F. X., \& Sousa, N. (2008). Lithium blocks stress-induced changes in depressive-like behavior and hippocampal cell fate: The role of glycogen-synthase-kinase-3ß. Neuroscience, 152(3), 656669. https://doi.org/10.1016/j.neuroscience.2007.12.026 
Simon, G. E., Goldberg, D. P., Korff, M. V., \& Üstün, T. B. (2002). Understanding cross-national differences in depression prevalence. Psychological Medicine, 32(4), 585-594. https://doi.org/10.1017/S0033291702005457

Slap, S. (2002). Application of microwave technology to histology. Theory and Practice of Histological Techniques, 5, 416.

Sloviter, R. S., \& Lømo, T. (2012). Updating the Lamellar Hypothesis of Hippocampal Organization. Frontiers in Neural Circuits, 6. https://doi.org/10.3389/fncir.2012.00102

Smits, B. M. G., Mudde, J. B., van de Belt, J., Verheul, M., Olivier, J., Homberg, J., Guryev, V., Cools, A. R., Ellenbroek, B. A., Plasterk, R. H. A., \& Cuppen, E. (2006). Generation of gene knockouts and mutant models in the laboratory rat by ENU-driven target-selected mutagenesis. Pharmacogenetics and Genomics, 16(3), 159-169. https://doi.org/10.1097/01.fpc.0000184960.82903.8f

Snyder, J. S., Ferrante, S. C., \& Cameron, H. A. (2012). Late Maturation of Adult-Born Neurons in the Temporal Dentate Gyrus. PLOS ONE, 7(11), e48757. https://doi.org/10.1371/journal.pone.0048757

Snyder, J. S., Hong, N. S., McDonald, R. J., \& Wojtowicz, J. M. (2005). A role for adult neurogenesis in spatial long-term memory. Neuroscience, 130(4), 843-852. https://doi.org/10.1016/j.neuroscience.2004.10.009

Snyder, J. S., Kee, N., \& Wojtowicz, J. M. (2001). Effects of Adult Neurogenesis on Synaptic Plasticity in the Rat Dentate Gyrus. Journal of Neurophysiology, 85(6), 2423-2431. https://doi.org/10.1152/jn.2001.85.6.2423

Snyder, J. S., Soumier, A., Brewer, M., Pickel, J., \& Cameron, H. A. (2011). Adult hippocampal neurogenesis buffers stress responses and depressive behaviour. Nature, 476(7361), 458-461. https://doi.org/10.1038/nature10287

Soiza-Reilly, M., Meye, F. J., Olusakin, J., Telley, L., Petit, E., Chen, X., Mameli, M., Jabaudon, D., Sze, J.-Y., \& Gaspar, P. (2019). SSRIs target prefrontal to raphe circuits during development modulating synaptic connectivity and emotional behavior. Molecular Psychiatry, 24(5), 726-745. https://doi.org/10.1038/s41380-018-0260-9

Soumier, A., Banasr, M., Goff, L. K.-L., \& Daszuta, A. (2010). Region- and phase-dependent effects of 5-HT1A and 5-HT2C receptor activation on adult neurogenesis. European Neuropsychopharmacology, 20(5), 336-345. https://doi.org/10.1016/j.euroneuro.2009.11.007 
Speranza, L., Labus, J., Volpicelli, F., Guseva, D., Lacivita, E., Leopoldo, M., Bellenchi, G. C., Porzio, U. di, Bijata, M., Perrone-Capano, C., \& Ponimaskin, E. (2017). Serotonin 5-HT7 receptor increases the density of dendritic spines and facilitates synaptogenesis in forebrain neurons. Journal of Neurochemistry, 141(5), 647-661. https://doi.org/10.1111/jnc.13962

Squire, L. R., Stark, C. E. L., \& Clark, R. E. (2004). The Medial Temporal Lobe. Annual Review of Neuroscience, 27(1), 279-306. https://doi.org/10.1146/annurev.neuro.27.070203.144130

Sripada, R. K., King, A. P., Garfinkel, S. N., Wang, X., Sripada, C. S., Welsh, R. C., \& Liberzon, I. (2012). Altered resting-state amygdala functional connectivity in men with posttraumatic stress disorder. Journal of Psychiatry \& Neuroscience : JPN, 37(4), 241-249. https://doi.org/10.1503/jpn.110069

Stepan, J., Dine, J., \& Eder, M. (2015). Functional optical probing of the hippocampal trisynaptic circuit in vitro: Network dynamics, filter properties, and polysynaptic induction of CA1 LTP. Frontiers in Neuroscience, 9. https://doi.org/10.3389/fnins.2015.00160

Stockmeier, C. A., Mahajan, G. J., Konick, L. C., Overholser, J. C., Jurjus, G. J., Meltzer, H. Y., Uylings, H. B. M., Friedman, L., \& Rajkowska, G. (2004). Cellular changes in the postmortem hippocampus in major depression. Biological Psychiatry, 56(9), 640-650. https://doi.org/10.1016/j.biopsych.2004.08.022

Stoltenberg, S. F., Twitchell, G. R., Hanna, G. L., Cook, E. H., Fitzgerald, H. E., Zucker, R. A., \& Little, K. Y. (2002). Serotonin transporter promoter polymorphism, peripheral indexes of serotonin function, and personality measures in families with alcoholism. American Journal of Medical Genetics, 114(2), 230-234. https://doi.org/10.1002/ajmg.10187

Suhonen, J. O., Peterson, D. A., Ray, J., \& Gage, F. H. (1996). Differentiation of adult hippocampus-derived progenitors into olfactory neurons in vivo. Nature, 383(6601), 624627. https://doi.org/10.1038/383624a0

Surget, A., Saxe, M., Leman, S., Ibarguen-Vargas, Y., Chalon, S., Griebel, G., Hen, R., \& Belzung, C. (2008). Drug-Dependent Requirement of Hippocampal Neurogenesis in a Model of Depression and of Antidepressant Reversal. Biological Psychiatry, 64(4), 293-301. https://doi.org/10.1016/j.biopsych.2008.02.022

Surtees, P. G., \& Barkley, C. (1994). Future Imperfect: The Long-Term Outcome of Depression. The British Journal of Psychiatry; London, 164(3), 327-341. http://dx.doi.org/10.1192/bjp.164.3.327 
Tamburella, A., Micale, V., Navarria, A., \& Drago, F. (2009). Antidepressant properties of the 5HT4 receptor partial agonist, SL65.0155: Behavioral and neurochemical studies in rats. Progress in Neuro-Psychopharmacology and Biological Psychiatry, 33(7), 1205-1210. https://doi.org/10.1016/j.pnpbp.2009.07.001

Tanaka, K., Waki, H., Ido, Y., Akita, S., Yoshida, Y., Yoshida, T., \& Matsuo, T. (1988). Protein and polymer analyses up to m/z 100000 by laser ionization time-of-flight mass spectrometry. Rapid Communications in Mass Spectrometry, 2(8), 151-153. https://doi.org/10.1002/rcm.1290020802

Tanti, A., \& Belzung, C. (2013). Neurogenesis along the septo-temporal axis of the hippocampus: Are depression and the action of antidepressants region-specific? Neuroscience, 252, 234 252. https://doi.org/10.1016/j.neuroscience.2013.08.017

Tau, G. Z., \& Peterson, B. S. (2010). Normal Development of Brain Circuits. Neuropsychopharmacology, 35(1), 147-168. https://doi.org/10.1038/npp.2009.115

Taupin, P. (2007). BrdU immunohistochemistry for studying adult neurogenesis: Paradigms, pitfalls, limitations, and validation. Brain Research Reviews, 53(1), 198-214. https://doi.org/10.1016/j.brainresrev.2006.08.002

Teicher, M. H., Anderson, C. M., \& Polcari, A. (2012). Childhood maltreatment is associated with reduced volume in the hippocampal subfields CA3, dentate gyrus, and subiculum. Proceedings of the National Academy of Sciences, 109(9), E563-E572. https://doi.org/10.1073/pnas.1115396109

Teissier, A., Soiza-Reilly, M., \& Gaspar, P. (2017). Refining the Role of 5-HT in Postnatal Development of Brain Circuits. Frontiers in Cellular Neuroscience, 11. https://doi.org/10.3389/fncel.2017.00139

Trachtenberg, J. T., Chen, B. E., Knott, G. W., Feng, G., Sanes, J. R., Welker, E., \& Svoboda, K. (2002). Long-term in vivo imaging of experience-dependent synaptic plasticity in adult cortex. Nature, 420(6917), 788-794. https://doi.org/10.1038/nature01273

Tran, P. V., Bymaster, F. P., McNamara, R. K., \& Potter, W. Z. (2003). Dual Monoamine Modulation for Improved Treatment of Major Depressive Disorder. Journal of Clinical Psychopharmacology, 23(1), 78-86.

Upton, A. L., Ravary, A., Salichon, N., Moessner, R., Lesch, K.-P., Hen, R., Seif, I., \& Gaspar, P. (2002). Lack of 5-HT1B receptor and of serotonin transporter have different effects on the 
segregation of retinal axons in the lateral geniculate nucleus compared to the superior colliculus. Neuroscience, 111(3), 597-610. https://doi.org/10.1016/S0306-4522(01)00602-9

Uytun, M. C. (2018). Development Period of Prefrontal Cortex. Prefrontal Cortex. https://doi.org/10.5772/intechopen.78697

Vadodaria, K. C., \& Jessberger, S. (2014). Functional neurogenesis in the adult hippocampus: Then and now. Frontiers in Neuroscience, 8. https://doi.org/10.3389/fnins.2014.00055

Vaidya, V. A., Fernandes, K., \& Jha, S. (2007). Regulation of adult hippocampal neurogenesis: Relevance to depression. Expert Review of Neurotherapeutics, 7(7), 853-864. https://doi.org/10.1586/14737175.7.7.853

van Hasselt, F. N., Cornelisse, S., Zhang, T. Y., Meaney, M. J., Velzing, E. H., Krugers, H. J., \& Joëls, M. (2012). Adult hippocampal glucocorticoid receptor expression and dentate synaptic plasticity correlate with maternal care received by individuals early in life. Hippocampus, 22(2), 255-266. https://doi.org/10.1002/hipo.20892

van Praag, H., Christie, B. R., Sejnowski, T. J., \& Gage, F. H. (1999). Running enhances neurogenesis, learning, and long-term potentiation in mice. Proceedings of the National Academy of Sciences, 96(23), 13427-13431. https://doi.org/10.1073/pnas.96.23.13427

van Praag, H. van, Schinder, A. F., Christie, B. R., Toni, N., Palmer, T. D., \& Gage, F. H. (2002). Functional neurogenesis in the adult hippocampus. Nature, 415(6875), 1030. https://doi.org/10.1038/4151030a

Vargha-Khadem, F., Gadian, D. G., Watkins, K. E., Connelly, A., Paesschen, W. V., \& Mishkin, M. (1997). Differential Effects of Early Hippocampal Pathology on Episodic and Semantic Memory. Science, 277(5324), 376-380. https://doi.org/10.1126/science.277.5324.376

Vázquez, D. M., Eskandari, R., Zimmer, C. A., Levine, S., \& López, J. F. (2002). Brain 5-HT receptor system in the stressed infant rat: Implications for vulnerability to substance abuse. Psychoneuroendocrinology, 27(1), 245-272. https://doi.org/10.1016/S0306-4530(01)00048-8

Vermetten, E., Vythilingam, M., Southwick, S. M., Charney, D. S., \& Bremner, J. D. (2003). Long-term treatment with paroxetine increases verbal declarative memory and hippocampal volume in posttraumatic stress disorder. Biological Psychiatry, 54(7), 693-702. https://doi.org/10.1016/S0006-3223(03)00634-6 
Vicentic, A., Francis, D., Moffett, M., Lakatos, A., Rogge, G., Hubert, G. W., Harley, J., \& Kuhar, M. J. (2006). Maternal separation alters serotonergic transporter densities and serotonergic 1A receptors in rat brain. Neuroscience, 140(1), 355-365.

https://doi.org/10.1016/j.neuroscience.2006.02.008

Videbech, P., \& Ravnkilde, B. (2004). Hippocampal volume and depression: A meta-analysis of MRI studies. The American Journal of Psychiatry, 161(11), 1957-1966.

https://doi.org/10.1176/appi.ajp.161.11.1957

Vitalis, T., Cases, O., Passemard, S., Callebert, J., \& Parnavelas, J. G. (2007). Embryonic depletion of serotonin affects cortical development. European Journal of Neuroscience, 26(2), 331-344. https://doi.org/10.1111/j.1460-9568.2007.05661.x

Vitalis, T., \& Parnavelas, J. G. (2003). The Role of Serotonin in Early Cortical Development. Developmental Neuroscience, 25(2-4), 245-256. https://doi.org/10.1159/000072272

Vollmayr, B., Simonis, C., Weber, S., Gass, P., \& Henn, F. (2003). Reduced cell proliferation in the dentate gyrusis not correlated with the development of learned helplessness. Biological Psychiatry, 54(10), 1035-1040. https://doi.org/10.1016/S0006-3223(03)00527-4

Vos, T., Allen, C., Arora, M., Barber, R. M., Bhutta, Z. A., Brown, A., Carter, A., Casey, D. C., Charlson, F. J., Chen, A. Z., Coggeshall, M., Cornaby, L., Dandona, L., Dicker, D. J., Dilegge, T., Erskine, H. E., Ferrari, A. J., Fitzmaurice, C., Fleming, T., ... Murray, C. J. L. (2016). Global, regional, and national incidence, prevalence, and years lived with disability for 310 diseases and injuries, 1990-2015: A systematic analysis for the Global Burden of Disease Study 2015. The Lancet, 388(10053), 1545-1602. https://doi.org/10.1016/S0140$\underline{6736(16) 31678-6}$

Vyas, A., Jadhav, S., \& Chattarji, S. (2006). Prolonged behavioral stress enhances synaptic connectivity in the basolateral amygdala. Neuroscience, 143(2), 387-393. https://doi.org/10.1016/j.neuroscience.2006.08.003

Wallace, J. A., \& Lauder, J. M. (1983). Development of the serotonergic system in the rat embryo: An immunocytochemical study. Brain Research Bulletin, 10(4), 459-479. https://doi.org/10.1016/0361-9230(83)90144-2

Wang, H., Zhao, Z., \& Guo, Y. (2013). Chemical and Biochemical Applications of MALDI TOFMS Based on Analyzing the Small Organic Compounds. In Z. Cai \& S. Liu (Eds.), 
Applications of MALDI-TOF Spectroscopy (pp. 165-192). Springer. https://doi.org/10.1007/128_2012_364

Wang, J.-W., David, D. J., Monckton, J. E., Battaglia, F., \& Hen, R. (2008). Chronic Fluoxetine Stimulates Maturation and Synaptic Plasticity of Adult-Born Hippocampal Granule Cells. Journal of Neuroscience, 28(6), 1374-1384. https://doi.org/10.1523/JNEUROSCI.3632$\underline{07.2008}$

Warner-Schmidt, J. L., \& Duman, R. S. (2006). Hippocampal neurogenesis: Opposing effects of stress and antidepressant treatment. Hippocampus, 16(3), 239-249. https://doi.org/10.1002/hipo.20156

Watanabe, Y., Sakai, R. R., McEwen, B. S., \& Mendelson, S. (1993). Stress and antidepressant effects on hippocampal and cortical 5-HT1A and 5-HT2 receptors and transport sites for serotonin. Brain Research, 615(1), 87-94. https://doi.org/10.1016/0006-8993(93)91117-B

Weissman, M. M., Bland, R. C., Canino, G. J., Faravelli, C., Greenwald, S., Hwu, H.-G., Joyce, P. R., Karam, E. G., Lee, C.-K., Lellouch, J., Lépine, J.-P., Newman, S. C., Rubio-Stipec, M., Wells, J. E., Wickramaratne, P. J., Wittchen, H.-U., \& Yeh, E.-K. (1996). Cross-National Epidemiology of Major Depression and Bipolar Disorder. JAMA, 276(4), 293-299. https://doi.org/10.1001/jama.1996.03540040037030

Wendland, J. R., Martin, B. J., Kruse, M. R., Lesch, K.-P., \& Murphy, D. L. (2006). Simultaneous genotyping of four functional loci of human SLC6A4, with a reappraisal of 5-HTTLPR and rs25531. Molecular Psychiatry, 11(3), 224-226. https://doi.org/10.1038/sj.mp.4001789

Whitaker-Azmitia, P. M., Murphy, R., \& Azmitia, E. C. (1990a). Stimulation of astroglial 5-HT1A receptors releases the serotonergic growth factor, protein S-100, and alters astroglial morphology. Brain Research, 528(1), 155-158. https://doi.org/10.1016/0006-8993(90)90210$\underline{3}$

Whitaker-Azmitia, P. M., Shemer, A. V., Caruso, J., Molino, L., \& Azmitia, E. C. (1990b). Role of High Affinity Serotonin Receptors in Neuronal Growtha. Annals of the New York Academy of Sciences, 600(1), 315-330. https://doi.org/10.1111/j.1749-6632.1990.tb16892.x

Whitaker-Azmitia, Patricia M., Druse, M., Walker, P., \& Lauder, J. M. (1995). Serotonin as a developmental signal. Behavioural Brain Research, 73(1), 19-29. https://doi.org/10.1016/0166-4328(96)00071-X 
Whooley, M. A., Kiefe, C. I., Chesney, M. A., Markovitz, J. H., Matthews, K., \& Hulley, S. B. (2002). Depressive Symptoms, Unemployment, and Loss of Income: The CARDIA Study. Archives of Internal Medicine, 162(22), 2614-2620.

https://doi.org/10.1001/archinte.162.22.2614

Wilbrecht, L., Holtmaat, A., Wright, N., Fox, K., \& Svoboda, K. (2010). Structural Plasticity Underlies Experience-Dependent Functional Plasticity of Cortical Circuits. Journal of Neuroscience, 30(14), 4927-4932. https://doi.org/10.1523/JNEUROSCI.6403-09.2010

Willner, P., \& Mitchell, P. J. (2002). The validity of animal models of predisposition to depression. Behavioural Pharmacology, 13(3), 169-188. https://doi.org/10.1097/00008877200205000-00001

Winocur, G., Wojtowicz, J. M., Sekeres, M., Snyder, J. S., \& Wang, S. (2006). Inhibition of neurogenesis interferes with hippocampus-dependent memory function. Hippocampus, 16(3), 296-304. https://doi.org/10.1002/hipo.20163

Winokur, S. B., Lopes, K. L., Moparthi, Y., \& Pereira, M. (2019). Depression-related disturbances in rat maternal behaviour are associated with altered monoamine levels within mesocorticolimbic structures. Journal of Neuroendocrinology, 31(9), e12766. https://doi.org/10.1111/jne.12766

Witteveen, J. S., Middelman, A., van Hulten, J. A., Martens, G. J. M., Homberg, J. R., \& Kolk, S. M. (2013). Lack of serotonin reuptake during brain development alters rostral rapheprefrontal network formation. Frontiers in Cellular Neuroscience, 7. https://doi.org/10.3389/fncel.2013.00143

Wong, M.-L., \& Licinio, J. (2001). Research and treatment approaches to depression. Nature Reviews Neuroscience, 2(5), 343-351. https://doi.org/10.1038/35072566

Woolley, C. S., Gould, E., \& McEwen, B. S. (1990). Exposure to excess glucocorticoids alters dendritic morphology of adult hippocampal pyramidal neurons. Brain Research, 531(1), 225231. https://doi.org/10.1016/0006-8993(90)90778-A

World Health Organization. (2017). Depression and other common mental disorders: global health estimates (No. WHO/MSD/MER/2017.2). World Health Organization.

Xia, L., Deloménie, C., David, I., Rainer, Q., Marouard, M., Delacroix, H., David, D. J., Gardier, A. M., \& Guilloux, J.-P. (2012). Ventral hippocampal molecular pathways and impaired 
neurogenesis associated with 5-HT1A and 5-HT1B receptors disruption in mice.

Neuroscience Letters, 521(1), 20-25. https://doi.org/10.1016/j.neulet.2012.05.046

Yamaguchi, S., Fujita, T., Fujino, T., \& Korenaga, T. (2008). Suppression of Matrix-related Ions Using Cyclodextrin in MALDI Mass Spectrometry. Analytical Sciences, 24(11), 1497-1500. https://doi.org/10.2116/analsci.24.1497

Yan, W., Wilson, C. C., \& Haring, J. H. (1997a). 5-HT1a receptors mediate the neurotrophic effect of serotonin on developing dentate granule cells. Developmental Brain Research, 98(2), 185-190. https://doi.org/10.1016/S0165-3806(96)00175-7

Yan, W., Wilson, C. C., \& Haring, J. H. (1997b). Effects of neonatal serotonin depletion on the development of rat dentate granule cells. Developmental Brain Research, 98(2), 177-184. https://doi.org/10.1016/S0165-3806(96)00176-9

Ye, H., Wang, J., Greer, T., Strupat, K., \& Li, L. (2013). Visualizing neurotransmitters and metabolites in the central nervous system by high resolution and high accuracy mass spectrometric imaging. ACS Chemical Neuroscience, 4(7), 1049-1056.

https://doi.org/10.1021/cn400065k

Zaikin, V. G., \& Halket, J. M. (2006). Derivatization in Mass Spectrometry-8. Soft Ionization Mass Spectrometry of Small Molecules. European Journal of Mass Spectrometry, 12(2), 79115. https://doi.org/10.1255/ejms.798

Zaverucha-do-Valle, C., Gubert, F., Mesentier-Louro, L., Scemes, E., Pitossi, F., Santiago, M. F., \& Mendez-Otero, R. (2013). Chapter 4-Resident Neural Stem Cells. In R. C. dos S. Goldenberg \& A. C. Campos de Carvalho (Eds.), Resident Stem Cells and Regenerative Therapy (pp. 69-87). Academic Press. https://doi.org/10.1016/B978-0-12-416012-5.00004-9

Zenobi, R., \& Knochenmuss, R. (1998). Ion formation in MALDI mass spectrometry. Mass Spectrometry Reviews, 17(5), 337-366. https://doi.org/10.1002/(SICI)10982787(1998)17:5<337::AID-MAS2>3.0.CO;2-S

Zeng, L.-L., Shen, H., Liu, L., Wang, L., Li, B., Fang, P., Zhou, Z., Li, Y., \& Hu, D. (2012). Identifying major depression using whole-brain functional connectivity: a multivariate pattern analysis. Brain, 135(5), 1498-1507. https://doi.org/10.1093/brain/aws059 
Zhang, C.-L., Zou, Y., He, W., Gage, F. H., \& Evans, R. M. (2008). A role for adult TLX-positive neural stem cells in learning and behaviour. Nature, 451(7181), 1004-1007. https://doi.org/10.1038/nature06562

Zhang, J., Cai, C.-Y., Wu, H.-Y., Zhu, L.-J., Luo, C.-X., \& Zhu, D.-Y. (2016). CREB-mediated synaptogenesis and neurogenesis is crucial for the role of 5-HT1a receptors in modulating anxiety behaviors. Scientific Reports, 6(1), 29551. https://doi.org/10.1038/srep29551

Zhao, C., Deng, W., \& Gage, F. H. (2008). Mechanisms and Functional Implications of Adult Neurogenesis. Cell, 132(4), 645-660. https://doi.org/10.1016/j.cell.2008.01.033

Zheng, H., Liu, Y., Li, W., Yang, B., Chen, D., Wang, X., Jiang, Z., Wang, H., Wang, Z., Cornelisson, G., \& Halberg, F. (2006). Beneficial effects of exercise and its molecular mechanisms on depression in rats. Behavioural Brain Research, 168(1), 47-55. https://doi.org/10.1016/j.bbr.2005.10.007

Zhou, F. C., Sari, Y., \& Zhang, J. K. (2000). Expression of serotonin transporter protein in developing rat brain. Brain Research. Developmental Brain Research, 119(1), 33-45. https://doi.org/10.1016/s0165-3806(99)00152-2

Zhuang, X., Gross, C., Santarelli, L., Compan, V., Trillat, A.-C., \& Hen, R. (1999). Altered Emotional States in Knockout Mice Lacking 5-HT1A or 5-HT1B Receptors. Neuropsychopharmacology, 21(1), 52-60. https://doi.org/10.1016/S0893-133X(99)00047-0

Zimmerman, T. A., Monroe, E. B., Tucker, K. R., Rubakhin, S. S., \& Sweedler, J. V. (2008). Imaging of Cells and Tissues with Mass Spectrometry: Adding Chemical Information to Imaging. In Methods in Cell Biology (Vol. 89, pp. 361-390). Academic Press. https://doi.org/10.1016/S0091-679X(08)00613-4 


\section{Appendix}

Table 1

Area means and SDs for each hippocampal subregion

\begin{tabular}{|c|c|c|c|c|c|c|c|c|}
\hline \multirow{2}{*}{$\begin{array}{l}\text { Offspring } \\
\text { genotype }\end{array}$} & \multirow{2}{*}{$\begin{array}{l}\text { Maternal } \\
\text { genotype }\end{array}$} & \multicolumn{2}{|c|}{ DG } & \multicolumn{2}{|c|}{ CA1 } & \multicolumn{2}{|c|}{$\mathrm{CA} 2+\mathrm{CA} 3$} & \multirow[b]{2}{*}{$n$} \\
\hline & & $M$ & $S D$ & $M$ & $S D$ & $M$ & $S D$ & \\
\hline \multirow[t]{2}{*}{ WT } & WT & 820.09 & 421.50 & 855.47 & 422.86 & 1241.70 & 530.74 & 4 \\
\hline & HET & 846.50 & 446.12 & 1030.02 & 433.70 & 1238.73 & 561.23 & 8 \\
\hline \multirow[t]{3}{*}{ HET } & WT & 848.24 & 337.49 & 754.14 & 311.57 & 1150.80 & 456.77 & 9 \\
\hline & HET & 787.60 & 364.99 & 885.71 & 386.28 & 1169.13 & 489.91 & 9 \\
\hline & $\mathrm{HOM}$ & 984.70 & 296 & 999 & 519.80 & 1427.40 & 378.73 & 4 \\
\hline \multirow[t]{2}{*}{$\mathrm{HOM}$} & HET & 817.02 & 381.27 & 919.91 & 333.02 & 931.20 & 392.02 & 3 \\
\hline & $\mathrm{HOM}$ & 838.63 & 381.04 & 993.80 & 461.15 & 1332.14 & 444.42 & 9 \\
\hline
\end{tabular}


Table 2

Two-way ANOVA for differences between offspring and maternal genotype in hippocampal subregion areas

\begin{tabular}{|c|c|c|c|c|c|c|}
\hline & & $\begin{array}{c}\text { Type IV sum of } \\
\text { squares }\end{array}$ & df & Mean square & $F$ & $p$-value \\
\hline \multirow[t]{5}{*}{ DG area } & Genotype & 46827.11 & 2 & 23413.56 & 0.164 & 0.849 \\
\hline & Maternal Genotype & 215277.95 & 2 & 107638.98 & 0.755 & 0.473 \\
\hline & $\begin{array}{l}\text { Genotype*Maternal } \\
\text { genotype }\end{array}$ & 109928.2 & 2 & 54964.100 & 0.385 & 0.681 \\
\hline & Error & 15688595.9 & 110 & 142623.60 & & \\
\hline & Total & 99696422.47 & 117 & & & \\
\hline \multirow[t]{5}{*}{ CA1 area } & Genotype & 104525.48 & 2 & 52262.74 & 0.303 & 0.739 \\
\hline & Maternal Genotype & 477311.23 & 2 & 238655.61 & 1.385 & 0.255 \\
\hline & $\begin{array}{l}\text { Genotype*Maternal } \\
\text { genotype }\end{array}$ & 9757.02 & 2 & 4878.51 & 0.028 & 0.972 \\
\hline & Error & 16373180.5 & 95 & 172349.27 & & \\
\hline & Total & 102775083 & 102 & & & \\
\hline \multirow{5}{*}{$\begin{array}{l}\mathrm{CA} 2+\mathrm{CA} 3 \\
\text { area }\end{array}$} & Genotype & 481749.87 & 2 & 240874.93 & 1.057 & 0.351 \\
\hline & Maternal Genotype & 1451273.10 & 2 & 725636.55 & 3.184 & 0.045 \\
\hline & $\begin{array}{l}\text { Genotype*Maternal } \\
\text { genotype }\end{array}$ & 60863.76 & 2 & 30431.88 & 0.134 & 0.875 \\
\hline & Error & 25069110.30 & 110 & 227901 & & \\
\hline & Total & 203980417 & 117 & & & \\
\hline
\end{tabular}

